Nickel(II)-Catalyzed Borylation of Alkenyl Methyl Ethers via C-O

\title{
Bond Cleavage
}

(Supporting Information)

Xiaodong Qiu,* Yangyang Li, Li Zhou, Peishan Chen, Fan Li, Yanan Zhang and Yong Ling*

School of Pharmacy, Nantong University, 19 Qixiu Road, Nantong 226001, China

Email: qiuxiaodong@ntu.edu.cn; Lyyy111@sina.com

\section{CONTENTS}

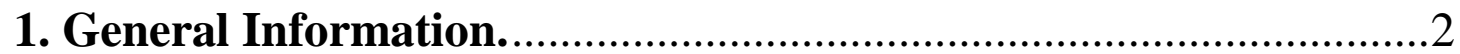

2. General Procedure for Synthesis of Enol Ethers ..............................2

3. General Procedure for Borylation of Alkenyl Methyl Ethers ..........9

4. Experimental Procedure for Gram-Scale Borylation of 1a...........15

5. Experimental Procedure for the Synthesis of Piceatannol (7) .......16

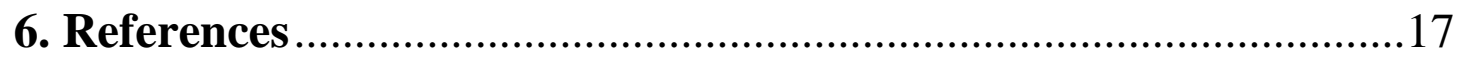

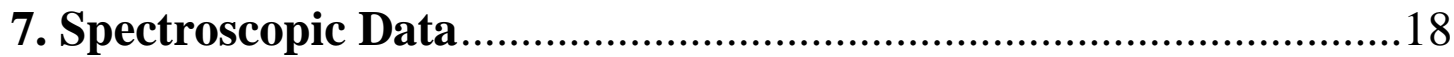




\section{General Information.}

Unless otherwise noted, all reactions were performed under an nitrogen atmosphere using flame-dried glassware. Toluene and THF were distilled over Na, DCE and acetonitrile were distilled over $\mathrm{CaH}_{2}$. Cyclohexane was purchased as anhydrous solvent and used directly. All new compounds were fully characterized. NMR-spectra were recorded on Bruker AV-300, ARX-400 MHz or a ARX-600 Associated. ${ }^{1} \mathrm{H}$ NMR spectra data were reported as $\delta$ values in ppm relative to chloroform $(\delta 7.26)$, methanol ( $\delta$ 3.30), or DMSO ( $\delta$ 2.50) if collected in $\mathrm{CDCl}_{3}, \mathrm{CD}_{3} \mathrm{OD}$, or DMSO-d ${ }^{6} \cdot{ }^{13} \mathrm{C}$ NMR spectra data were reported as $\delta$ values in ppm relative to chloroform $(\delta$ 77.0) methanol $(\delta 49.0)$ or DMSO $(\delta$ 39.5 ) if collected in $\mathrm{CDCl}_{3}$ (the carbon attached to B was not observed), $\mathrm{CD}_{3} \mathrm{OD}$, DMSO-d $\mathrm{d}^{6} .{ }^{1} \mathrm{H}$ NMR coupling constants were reported in $\mathrm{Hz}$, and multiplicity was indicated as follows: $\mathrm{s}$ (singlet); d (doublet); $\mathrm{t}$ (triplet); q (quartet); quint (quintet); $\mathrm{m}$ (multiplet); dd (doubletof doublets); ddd (doublet of doublet of doublets); dddd (doublet of doublet of doublet of doublets); dt (doublet of triplets); td (triplet of doublets); ddt (doublet of doublet of triplets); dq (doubletof quartets); app (apparent); br (broad). Mass spectra were conducted at Micromass Q-Tof instrument (ESI) and Agilent Technologies 5973N (EI). All reactions were carried out in flame-dried $25 \mathrm{~mL}$ Schlenk tubes with Teflon screw caps under nitrogen. $\mathrm{B}_{2} \mathrm{Pin}_{2}$ was vacuumized under room temperature for $12 \mathrm{~h}$ before use. Unless otherwise noted, materials obtained from commercial suppliers were used without further purification. Compounds $1 \mathbf{a},{ }^{\mathrm{S} 1} \mathbf{1 b},{ }^{\mathrm{S} 2} \mathbf{1 d},{ }^{\mathrm{S} 3}$ $\mathbf{1 f},{ }^{\mathrm{S} 1} \mathbf{1 g},{ }^{\mathrm{S} 1} \mathbf{1} \mathbf{j},{ }^{\mathrm{S} 2} 1 \mathbf{l},{ }^{\mathrm{S} 4} \mathbf{1 m},{ }^{\mathrm{S} 5} \mathbf{1} \mathbf{p},{ }^{\mathrm{S} 5} 1 \mathbf{r},{ }^{\mathrm{S} 1} 1 \mathbf{s}^{\mathrm{S} 6}$ were prepared according to litriture reports.

\section{General Procedure for Synthesis of Enol Ethers}

$$
\begin{aligned}
& \mathrm{R}-\mathrm{CHO}+\mathrm{MeO} \widehat{\mathrm{PPh}_{3}^{+}} \underset{\mathrm{THF}, 0^{\circ} \mathrm{C}-\mathrm{rt}}{\stackrel{t-\mathrm{BuOK}}{\mathrm{C}}} \stackrel{\mathrm{R}}{{ }_{1}^{m} \mathrm{OMe}} \\
& E / Z
\end{aligned}
$$

To a flame dried flask was added (methoxymethyl)triphenylphosphonium chloride ( $7.5 \mathrm{mmol}, 2.57 \mathrm{~g}$ ) and $t$-BuOK $(9.0 \mathrm{mmol}, 1.01 \mathrm{~g})$, the flask was vacuumed and refilled with nitrogen three times and put under the ice bath. $15 \mathrm{~mL}$ anhydrous THF was added and the mixture was stirred for $30 \mathrm{~min}$. After that, the solution of aldehyde $(5.0 \mathrm{mmol})$ in THF $(5 \mathrm{~mL})$ was added dropwise into the reaction mixturre, then the reaction was stirred under room temperature overnight. The reaction was monitored by TLC. After the aldehyde was dispeared, $30 \mathrm{~mL}$ water was added and stirred for another $5 \mathrm{~min}$. The aqueous phase 
was extracted by ethyl acetate and the combined organic phase was dried over anhydrous sodium sulfate. The mixture was then filtered and the solvent was removed under reduced pressure. Further purification through flash chromatography $(\mathrm{PE}: \mathrm{EA}=50: 1)$ would prodive the product 1 as a mixture with both $E$ and $Z$ isomers.

\section{1-(2-Methoxyvinyl)-3-methylbenzene (1c)}<smiles>COC=Cc1cccc(C)c1</smiles>

$E / Z$
Compound 1c was prepared from 3-methylbenzaldehyde in $92 \%$ yield as a colorless oil $(680 \mathrm{mg})$. The spectral data were given for the mixture of both (E)-1c and $(Z)-\mathbf{1 c}(E: Z=51: 49) .{ }^{1} \mathrm{H}$ NMR $\left(400 \mathrm{MHz}, \mathrm{CDCl}_{3}\right) \delta 7.39(\mathrm{~d}, J=$

$6.4 \mathrm{~Hz}, 2 \mathrm{H}), 7.23-7.13(\mathrm{~m}, 2 \mathrm{H}), 7.09-7.01(\mathrm{~m}, 3 \mathrm{H}), 7.00-6.94(\mathrm{~m}, 2 \mathrm{H}), 6.13(\mathrm{~d}, J=7.0 \mathrm{~Hz}, 1 \mathrm{H})$, $5.79(\mathrm{~d}, J=12.9 \mathrm{~Hz}, 1 \mathrm{H}), 5.20(\mathrm{~d}, J=7.0 \mathrm{~Hz}, 1 \mathrm{H}), 3.79(\mathrm{~s}, 3 \mathrm{H}), 3.69$ (s, 3H), $2.34(\mathrm{~s}, 3 \mathrm{H}), 2.33(\mathrm{~s}, 3 \mathrm{H})$. ${ }^{13} \mathrm{C}$ NMR $\left(101 \mathrm{MHz}, \mathrm{CDCl}_{3}\right) \delta 148.7,147.8,138.1,137.6,136.2,135.7,128.8,128.5,128.1,126.5$, 126.4, 125.9, 125.3, 122.2, 105.7, 105.0, 60.6, 56.5, 21.5, 21.4. ATR-FTIR (cm $\left.{ }^{-1}\right): 1421,1262,896,733$, 703. HRMS m/z (ESI) calcd for $\mathrm{C}_{10} \mathrm{H}_{13} \mathrm{O}(\mathrm{M}+\mathrm{H})^{+}$149.0961, found 149.0960 .

\section{1-(tert-Butyl)-4-(2-methoxyvinyl)benzene (1e)}<smiles>COC=Cc1ccc(C(C)(C)C)cc1</smiles>

$E / Z$

Compound 1e was prepared from 4-(tert-butyl)benzaldehyde in 95\% yield as a colorless oil (906 mg). The spectral data were given for the mixture of both $(E)-\mathbf{1 e}$ and $(Z)-\mathbf{1 e}(E: Z=39: 61) .{ }^{1} \mathrm{H}$ NMR $(400 \mathrm{MHz}$,

$\left.\mathrm{CDCl}_{3}\right) \delta 7.55-7.45(\mathrm{~m}, 2 \mathrm{H}), 7.36-7.24(\mathrm{~m}, 3.28 \mathrm{H}), 7.21-7.13(\mathrm{~m}, 1.49 \mathrm{H}), 7.01(\mathrm{~d}, J=13.0 \mathrm{~Hz}$, $0.63 \mathrm{H}), 6.08(\mathrm{~d}, J=6.9 \mathrm{~Hz}, 1 \mathrm{H}), 5.79(\mathrm{~d}, J=13.0 \mathrm{~Hz}, 0.63 \mathrm{H}), 5.20(\mathrm{~d}, J=7.0 \mathrm{~Hz}, 1 \mathrm{H}), 3.73(\mathrm{~s}, 3 \mathrm{H})$, $3.65(\mathrm{~s}, 2 \mathrm{H}), 1.30(\mathrm{~s}, 15.75 \mathrm{H}) .{ }^{13} \mathrm{C} \mathrm{NMR}\left(101 \mathrm{MHz}, \mathrm{CDCl}_{3}\right) \delta 148.53,148.52,148.3,147.3,133.4,133.0$, 127.8, 125.5, 125.0, 124.8, 105.4, 104.7, 60.5, 56.4, 34.4, 34.3, 31.29, 31.28. ATR-FTIR ( $\left.\mathrm{cm}^{-1}\right): 1642$, 1264, 1097, 895, 841, 733, 702. HRMS m/z (ESI) calcd for $\mathrm{C}_{13} \mathrm{H}_{19} \mathrm{O}(\mathrm{M}+\mathrm{H})^{+}$191.1430, found 191.1429.

\section{4-(2-Methoxyvinyl)-1,1'-biphenyl (1h)}<smiles>COC=Cc1ccc(-c2ccccc2)cc1</smiles>

$E / Z$

Compound $\mathbf{1 h}$ was prepared from [1,1'-biphenyl]-4-carbaldehyde in $94 \%$ yield as a white solid (985 mg). The spectral data were given for the mixture of both $(E)-\mathbf{1 h}$ and $(Z)-\mathbf{1 h}(E: Z=87: 13) .{ }^{1} \mathrm{H}$ NMR $\left(400 \mathrm{MHz}, \mathrm{CDCl}_{3}\right) \delta 7.68$

$-7.57(\mathrm{~m}, 2.50 \mathrm{H}), 7.56-7.49(\mathrm{~m}, 2.26 \mathrm{H}), 7.56-7.49(\mathrm{~m}, 2.26 \mathrm{H}), 7.47-7.37(\mathrm{~m}, 2.23 \mathrm{H}), 7.36-7.28$ 
$(\mathrm{m}, 3.13 \mathrm{H}), 7.11(\mathrm{~d}, J=13.0 \mathrm{~Hz}, 1 \mathrm{H}), 6.18(\mathrm{~d}, J=7.0 \mathrm{~Hz}, 0.15 \mathrm{H}), 5.86(\mathrm{~d}, J=13.0 \mathrm{~Hz}, 1 \mathrm{H}), 5.28(\mathrm{~d}, J$ $=7.0 \mathrm{~Hz}, 0.15 \mathrm{H}), 3.81(\mathrm{~s}, 0.40 \mathrm{H}), 3.72(\mathrm{~s}, 3 \mathrm{H}) .{ }^{13} \mathrm{C} \mathrm{NMR}\left(101 \mathrm{MHz}, \mathrm{CDCl}_{3}\right) \delta 149.0,148.2,141.1,140.8$, $138.4,138.3,135.4,135.0,128.72,128.68,128.5,127.3,126.99,126.95,126.9,126.83,126.76,125.4$, 105.2, 104.6, 60.7, 56.6. ATR-FTIR ( $\left.\mathrm{cm}^{-1}\right):$ 3030, 2930, 1635, 1482, 1243, 1149, 1087, 95, 843, 760, 693. HRMS m/z (ESI) calcd for $\mathrm{C}_{15} \mathrm{H}_{15} \mathrm{O}(\mathrm{M}+\mathrm{H})^{+} 211.1117$, found 211.1116.

\section{(4-(2-Methoxyvinyl)phenyl)(methyl)sulfane (1i)}<smiles>[Z7]#[Cr]c1ccc(C=COC)cc1</smiles>

Compound 1i was prepared from 4-(methylthio)benzaldehyde in 90\% yield as a colorless oil $(813 \mathrm{mg})$. The spectral data were given for the mixture of both $(E)-1 \mathbf{i}$ and $(Z)-1 \mathrm{i}(E: Z=58: 42) .{ }^{1} \mathrm{H}$ NMR $(400 \mathrm{MHz}$,

$\left.\mathrm{CDCl}_{3}\right) \delta 7.50(\mathrm{~d}, J=8.5 \mathrm{~Hz}, 1.44 \mathrm{H}), 7.23-7.11(\mathrm{~m}, 5.42 \mathrm{H}), 7.03(\mathrm{~d}, J=13.0 \mathrm{~Hz}, 1 \mathrm{H}), 6.12(\mathrm{~d}, J=6.9$ $\mathrm{Hz}, 0.71 \mathrm{H}), 5.77(\mathrm{~d}, J=13.0 \mathrm{~Hz}, 1 \mathrm{H}), 5.19(\mathrm{~d}, J=7.0 \mathrm{~Hz}, 0.71 \mathrm{H}), 3.78(\mathrm{~s}, 2.13 \mathrm{H}), 3.68(\mathrm{~s}, 3 \mathrm{H}), 2.474$ (s, 2.17H), $2.471(\mathrm{~s}, 3 \mathrm{H}) .{ }^{13} \mathrm{C} \mathrm{NMR}\left(101 \mathrm{MHz}, \mathrm{CDCl}_{3}\right) \delta 148.6,147.7,135.1,135.0,133.5,133.0,128.6$, 127.4, 126.7, 125.5, 105.1, 104.5, 60.7, 56.5, 16.4, 16.2. ATR-FTIR $\left(\mathrm{cm}^{-1}\right): 1423,1265,898,734,701$. HRMS m/z (ESI) calcd for $\mathrm{C}_{10} \mathrm{H}_{13} \mathrm{OS}(\mathrm{M}+\mathrm{H})^{+}$181.0682, found 181.0681 .

\section{1-Fluoro-2-(2-methoxyvinyl)benzene (1k)}<smiles>COC=Cc1ccccc1F</smiles>

Compound $\mathbf{1 k}$ was prepared from 2-fluorobenzaldehyde in $87 \%$ yield as a colorless oil (662 mg). The spectral data were given for the mixture of both $(E)$ $1 \mathbf{k}$ and $(Z)-1 \mathbf{k}(E: Z=60: 40) .{ }^{1} \mathrm{H} \mathrm{NMR}\left(400 \mathrm{MHz}, \mathrm{CDCl}_{3}\right) \delta 8.05(\mathrm{td}, J=7.7,2.5$ $\mathrm{Hz}, 0.66 \mathrm{H}), 7.33-7.23(\mathrm{~m}, 1 \mathrm{H}), 7.18(\mathrm{~d}, J=13.0 \mathrm{~Hz}, 1 \mathrm{H}), 7.15-6.96(\mathrm{~m}, 4.95 \mathrm{H})$,

$6.24(\mathrm{~d}, J=7.1 \mathrm{~Hz}, 0.67 \mathrm{H}), 5.88(\mathrm{~d}, J=13.1 \mathrm{~Hz}, 1 \mathrm{H}), 5.50(\mathrm{~d}, J=7.0 \mathrm{~Hz}, 0.67 \mathrm{H}), 3.80(\mathrm{~s}, 2.09 \mathrm{H}), 3.72$ (s, 3H). ${ }^{13} \mathrm{C}$ NMR $\left(101 \mathrm{MHz}, \mathrm{CDCl}_{3}\right) \delta 160.4(\mathrm{~d}, \mathrm{~J}=39.6 \mathrm{~Hz}), 158.0(\mathrm{~d}, \mathrm{~J}=40.7 \mathrm{~Hz}), 150.7(\mathrm{~d}, \mathrm{~J}=6.6$ Hz), 149.1 (d, J = 2.2 Hz), $130.0(\mathrm{~d}, \mathrm{~J}=3.3 \mathrm{~Hz}), 126.9$ (d, J = 8.3 Hz), 126.6 (d, J = 8.2 Hz), 126.5 (d, J $=4.4 \mathrm{~Hz}), 124.1,124.0(\mathrm{~d}, \mathrm{~J}=3.4 \mathrm{~Hz}), 124.0,123.7(\mathrm{~d}, \mathrm{~J}=3.6 \mathrm{~Hz}), 123.6(\mathrm{~d}, \mathrm{~J}=11.9 \mathrm{~Hz}), 115.5(\mathrm{~d}, \mathrm{~J}=$ 22.2 Hz), $114.7(\mathrm{~d}, \mathrm{~J}=22.2 \mathrm{~Hz}), 98.2(\mathrm{~d}, \mathrm{~J}=2.8 \mathrm{~Hz}), 96.4(\mathrm{~d}, \mathrm{~J}=7.6 \mathrm{~Hz}), 60.7,56.4 .{ }^{19} \mathrm{~F}$ NMR $(376$ $\left.\mathrm{MHz}, \mathrm{CDCl}_{3}\right) \delta-118.8,-117.5$. ATR-FTIR $\left(\mathrm{cm}^{-1}\right)$ : 1420, 1262, 894, 732, 702. HRMS m/z (ESI) calcd for $\mathrm{C}_{9} \mathrm{H}_{10} \mathrm{FO}(\mathrm{M}+\mathrm{H})^{+}$153.0710, found 153.0713. 
<smiles>CCOC(=O)c1ccc(/C=C/OC)cc1</smiles>

Compound 1n was prepared from $N, N$-diethyl-4-formylbenzamide in $85 \%$ yield as a colorless oil $(995 \mathrm{mg})$. The spectral data were given for the mixture of both $(E)-\mathbf{1 n}$ and $(Z)-\mathbf{1 n}(E: Z=36: 64) .{ }^{1} \mathrm{H}$ NMR $\left(400 \mathrm{MHz}, \mathrm{CDCl}_{3}\right) \delta 7.62-7.52(\mathrm{~m}, 2 \mathrm{H}), 7.35-7.26(\mathrm{~m}, 3.61 \mathrm{H})$, $7.26-7.21(\mathrm{~m}, 1.14 \mathrm{H}), 7.09(\mathrm{~d}, J=13.0 \mathrm{~Hz}, 0.58 \mathrm{H}), 6.18(\mathrm{~d}, J=7.0 \mathrm{~Hz}, 1 \mathrm{H}), 5.80(\mathrm{~d}, J=13.0 \mathrm{~Hz}$ $0.56 \mathrm{H}), 5.23(\mathrm{~d}, J=7.0 \mathrm{~Hz}, 1 \mathrm{H}), 3.80(\mathrm{~s}, 3 \mathrm{H}), 3.70(\mathrm{~s}, 1.69 \mathrm{H}), 3.65-3.10(\mathrm{~m}, 6.45 \mathrm{H}), 1.35-0.97(\mathrm{~m}$, 9.98H). ${ }^{13} \mathrm{C}$ NMR $\left(101 \mathrm{MHz}, \mathrm{CDCl}_{3}\right) \delta 171.4,171.2,149.6,148.7,137.4,136.8,134.2,134.1,127.9$, 126.8, 126.3, 124.8, 104.9, 104.3, 60.8, 56.5, 43.2, 39.2, 14.2, 12.9. ATR-FTIR ( $\left.\mathrm{cm}^{-1}\right): 1624,1424,1263$, 1095, 895, 731, 702. HRMS m/z (ESI) calcd for $\mathrm{C}_{14} \mathrm{H}_{20} \mathrm{NO}_{2}(\mathrm{M}+\mathrm{H})^{+}$234.1489, found 234.1488.

\section{1-(2-Methoxyvinyl)-3,5-dimethylbenzene (10)}

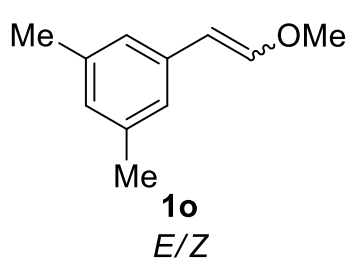

Compound 10 was prepared from 3,5-dimethylbenzaldehyde in 95\% yield as a colorless oil $(770 \mathrm{mg})$. The spectral data were given for the mixture of both $(E)-10$ and $(Z)-10(E: Z=50: 50) .{ }^{1} \mathrm{H}$ NMR $\left(400 \mathrm{MHz}, \mathrm{CDCl}_{3}\right) \delta 7.22(\mathrm{~s}$, 2H), $7.05(\mathrm{~d}, J=13.0 \mathrm{~Hz}, 1 \mathrm{H}), 6.93-6.75(\mathrm{~m}, 4 \mathrm{H}), 6.11(\mathrm{~d}, J=7.0 \mathrm{~Hz}, 1 \mathrm{H})$, $5.78(\mathrm{~d}, J=13.0 \mathrm{~Hz}, 1 \mathrm{H}), 5.18(\mathrm{~d}, J=7.0 \mathrm{~Hz}, 1 \mathrm{H}), 3.79(\mathrm{~s}, 3 \mathrm{H}), 3.69(\mathrm{~s}, 3 \mathrm{H}), 2.36-2.22(\mathrm{~m}, 12 \mathrm{H}) .{ }^{13} \mathrm{C}$ $\operatorname{NMR}\left(101 \mathrm{MHz}, \mathrm{CDCl}_{3}\right) \delta 148.6,147.6,138.0,137.5,136.1,135.6,127.5,127.4,126.0,123.0,105.7$, 105.0, 60.6, 56.4, 21.3, 21.3. ATR-FTIR $\left(\mathrm{cm}^{-1}\right): 1421,1264,1100,895,731,702$. HRMS m/z (ESI) calcd for $\mathrm{C}_{11} \mathrm{H}_{15} \mathrm{O}(\mathrm{M}+\mathrm{H})^{+}$163.1117, found 163.1117 .

\section{5-(2-Methoxyvinyl)-2,3-dihydrobenzofuran (1q)}

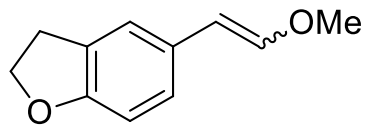

$1 q$

$E / Z$

Compound $\mathbf{1 q}$ was prepared from 2,3-dihydrobenzofuran-5carbaldehyde in $94 \%$ yield as a colorless oil $(825 \mathrm{mg})$. The spectral data were given for the mixture of both $(E)-\mathbf{1 q}$ and $(Z)-\mathbf{1 q}(E: Z=45: 55) .{ }^{1} \mathrm{H}$ $\operatorname{NMR}\left(400 \mathrm{MHz}, \mathrm{CDCl}_{3}\right) \delta 7.55(\mathrm{~s}, 1 \mathrm{H}), 7.29(\mathrm{~d}, J=8.2 \mathrm{~Hz}, 1 \mathrm{H}), 7.11(\mathrm{~s}$, $0.81 \mathrm{H}), 6.99(\mathrm{~d}, J=8.2 \mathrm{~Hz}, 0.80 \mathrm{H}), 6.93(\mathrm{~d}, J=13.0 \mathrm{~Hz}, 0.81 \mathrm{H}), 6.78-6.68(\mathrm{~m}, 1.77 \mathrm{H}), 6.04(\mathrm{~d}, J=$ $7.0 \mathrm{~Hz}, 1 \mathrm{H}), 5.80(\mathrm{~d}, J=13.0 \mathrm{~Hz}, 0.81 \mathrm{H}), 5.19(\mathrm{~d}, J=7.0 \mathrm{~Hz}, 1 \mathrm{H}), 4.63-4.50(\mathrm{~m}, 3.74 \mathrm{H}), 3.76(\mathrm{~s}, 3 \mathrm{H})$, $3.67(\mathrm{~s}, 2.43 \mathrm{H}), 3.24-3.13(\mathrm{~m}, 3.78 \mathrm{H}) .{ }^{13} \mathrm{C} \mathrm{NMR}\left(101 \mathrm{MHz}, \mathrm{CDCl}_{3}\right) \delta 158.3,158.0,147.0,145.7,128.6$, $128.5,128.0,127.3,126.7,125.0,124.7,121.3,109.1,108.7,105.5,104.9,71.1,71.1,60.3,56.3,29.6$, 29.6. ATR-FTIR $\left(\mathrm{cm}^{-1}\right): 1646,1490,1264,1106,984,735,702$. HRMS m/z (ESI) calcd for $\mathrm{C}_{11} \mathrm{H}_{13} \mathrm{O}_{2}$ 


\section{9-Ethyl-3-(2-methoxyvinyl)-9H-carbazole (1t)}

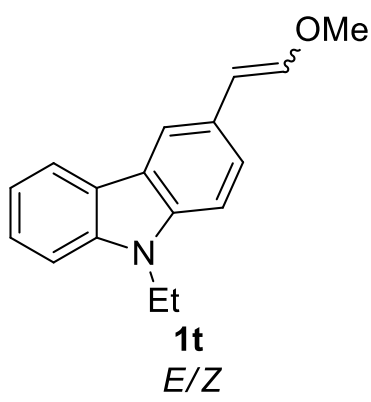

Compound 1t was prepared from 9-ethyl-9H-carbazole-3-carbaldehyde in $86 \%$ yield as a pale yellow solid (1.08 g). The spectral data were given for the mixture of both $(E)-\mathbf{1 t}$ and $(Z)-\mathbf{1 t}(E: Z=50: 50) .{ }^{1} \mathrm{H} \mathrm{NMR}(400 \mathrm{MHz}$, $\left.\mathrm{CDCl}_{3}\right) \delta 8.36-8.27(\mathrm{~m}, 1 \mathrm{H}), 8.15-7.96(\mathrm{~m}, 2 \mathrm{H}), 7.95-7.85(\mathrm{~m}, 1 \mathrm{H})$, $7.74-7.63(\mathrm{~m}, 1 \mathrm{H}), 7.43-7.32(\mathrm{~m}, 2 \mathrm{H}), 7.31-7.10(\mathrm{~m}, 7 \mathrm{H}), 7.09-6.99$ $(\mathrm{m}, 1 \mathrm{H}), 6.08-5.91(\mathrm{~m}, 2 \mathrm{H}), 5.43-5.30(\mathrm{~m}, 1 \mathrm{H}), 4.22-4.04(\mathrm{~m}, 4 \mathrm{H})$, $3.71(\mathrm{~s}, 3 \mathrm{H}), 3.63(\mathrm{~s}, 3 \mathrm{H}), 1.34-1.14(\mathrm{~m}, 6 \mathrm{H}) .{ }^{13} \mathrm{C} \mathrm{NMR}\left(101 \mathrm{MHz}, \mathrm{CDCl}_{3}\right) \delta 147.2,145.9,140.2,138.5$, $138.3,127.1,127.0,126.5,125.6,125.4,123.3,123.2,123.1,122.9,122.8,120.5,120.4,120.0,118.61$, $118.55,116.9,108.6,108.43,108.35,108.0,106.4,105.9,60.5,56.5,37.5,37.5,13.8$. ATR-FTIR $\left(\mathrm{cm}^{-1}\right)$ : 1421, 1267, 894, 734, 703. HRMS m/z (ESI) calcd for $\mathrm{C}_{17} \mathrm{H}_{18} \mathrm{NO}_{2}(\mathrm{M}+\mathrm{H})^{+}$252.1383, found 252.1383.

\section{1-(2-Methoxyvinyl)cyclohex-1-ene (1v)}

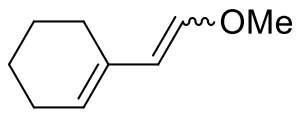

$1 \mathrm{v}$

$E / Z$

Compound 1v was prepared from cyclohex-1-enecarbaldehyde in $68 \%$ yield as a colorless oil (469 $\mathrm{mg})$. Due to the instability of $\mathbf{1 v}$ under room temperature, it was put into the borylation reaction immediately after purification by column chromatography on silica gel. The NMR spectral data of $\mathbf{1 v}$ were deficient but it could be easily judged from GC-MS that $\mathbf{1 v}$ was the mixture of $E / Z$ isomers (42:58). EI-MS (m/z, relative intensity, $\mathrm{rt}=5.118$ $\min ): 138\left(\mathrm{M}^{+}, 100\right), 123$ (36), 109 (52), 106 (20), 95 (45), 91 (42), 79 (56), 67 (31), 53(14). EI-MS (m/z, relative intensity, $\mathrm{rt}=5.158 \mathrm{~min}): 138\left(\mathrm{M}^{+}, 100\right), 123(35), 109(50), 106(19), 95(43), 91(41), 79(54)$, 67 (30), 53(13). EI-MS (m/z, relative intensity, rt = $5.456 \mathrm{~min}): 138\left(\mathrm{M}^{+}, 100\right), 123(40), 109(54), 106$ (21), 95 (49), 91 (46), 79 (59), 67 (33), 53(14). EI-MS (m/z, relative intensity, $\mathrm{rt}=5.479 \mathrm{~min}): 138\left(\mathrm{M}^{+}\right.$, 100), 123 (39), 109 (52), 106 (20), 95 (47), 91 (44), 79 (58), 67 (32), 53(14). ATR-FTIR (cm-1): 1266, 1091, 894, 734, 704. HRMS m/z (ESI) calcd for $\mathrm{C}_{9} \mathrm{H}_{15} \mathrm{O}(\mathrm{M}+\mathrm{H})^{+} 139.1117$, found 139.1117 . 


\section{Chromatogram Plot Report}

Agilent

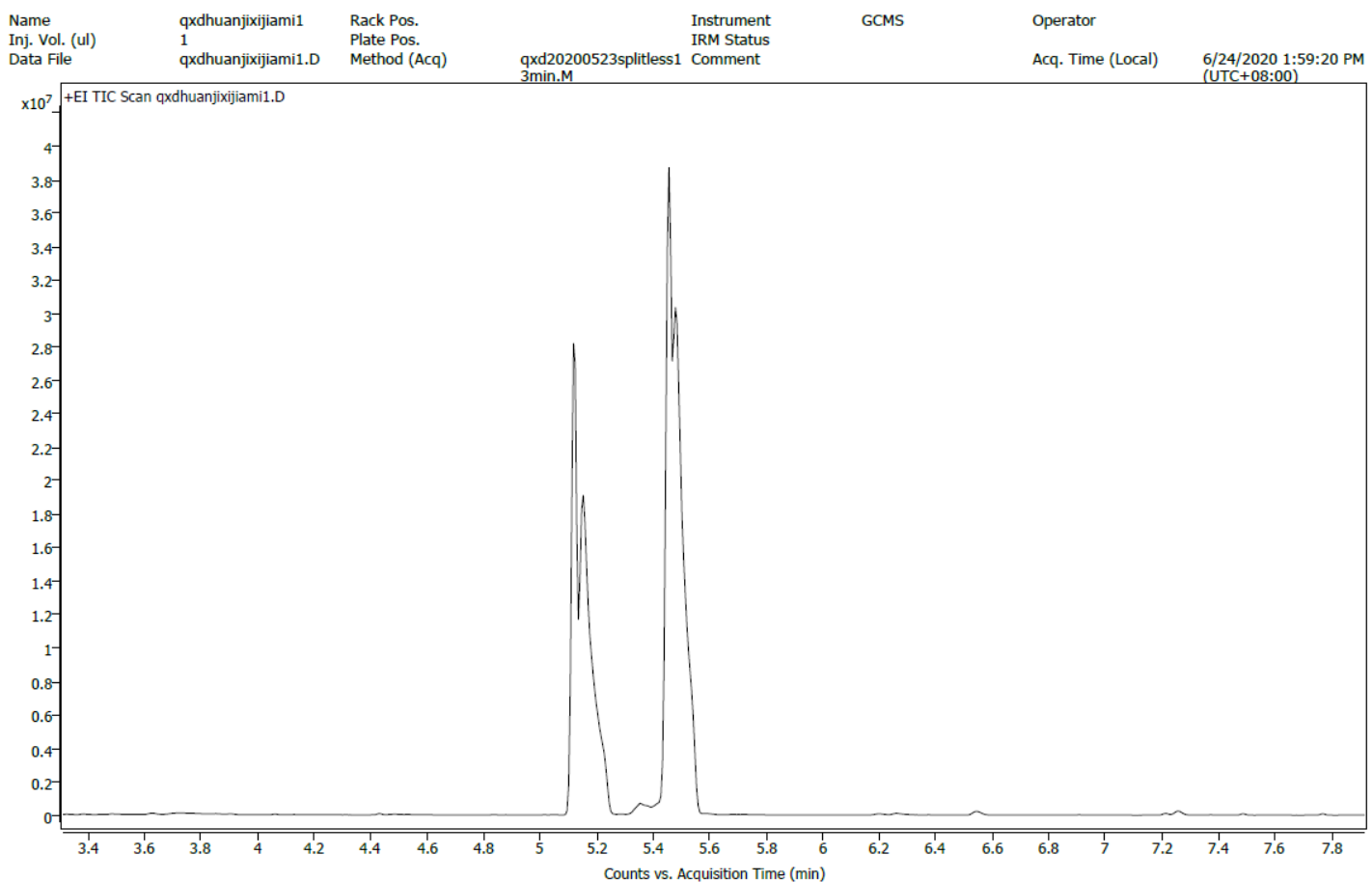

Page 1 of 1

Generated at 3:08 PM on 6/24/2020

\section{(S)-1-(2-methoxyvinyl)-4-(prop-1-en-2-yl)cyclohex-1-ene (1w)}

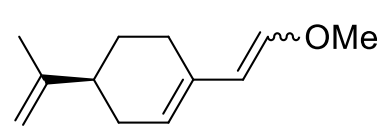

$1 w$

$E / Z$

Compound 1w was prepared from (-)-perillaldehyde in $60 \%$ yield as a colorless oil $(535 \mathrm{mg})$. Due to the instability of $\mathbf{1 w}$ under room temperature, it was put into the borylation reaction immediately after purification by column chromatography on silica gel. The NMR spectral data of $\mathbf{1 w}$ were deficient but it could be easily judged from GC-MS that two isomers existed (47:53). EI-MS (m/z, relative intensity, $\mathrm{rt}=7.436 \mathrm{~min}): 178\left(\mathrm{M}^{+}, 36\right), 137(91), 110(100), 95(85), 91(28), 79(47), 67(35)$, 53(13). EI-MS (m/z, relative intensity, $r t=7.728 \mathrm{~min}): 178\left(\mathrm{M}^{+}, 37\right), 137(91), 110(100), 95(87), 91$ (29), 79 (49), 67 (37), 53(14). ATR-FTIR ( $\left.\mathrm{cm}^{-1}\right):$ 1649, 1623, 1267, 1091, 893, 733, 704. HRMS m/z (ESI) calcd for $\mathrm{C}_{12} \mathrm{H}_{19} \mathrm{O}(\mathrm{M}+\mathrm{H})^{+} 179.1430$, found 179.1429 . 


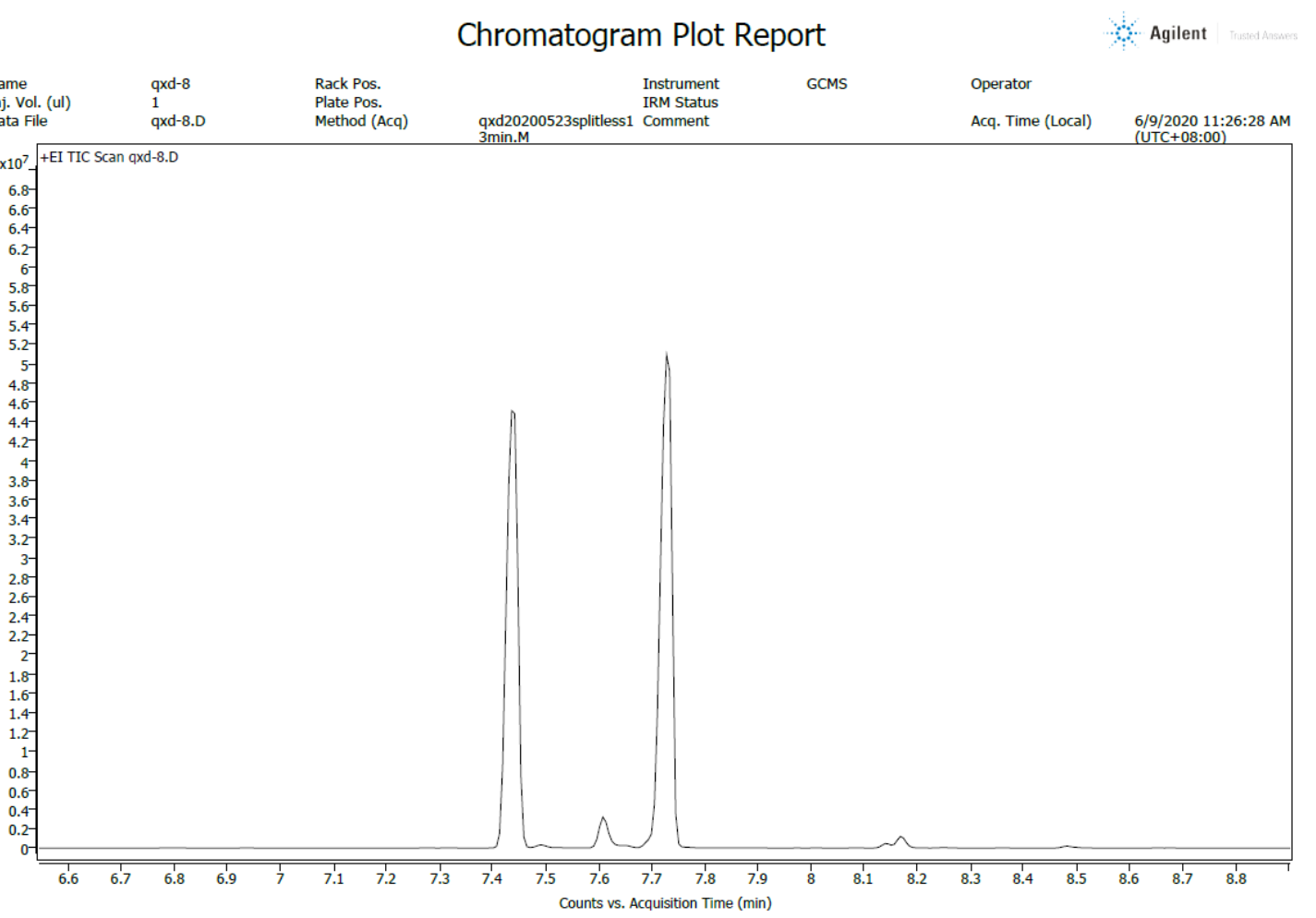

Page 1 of 1

Generated at 3.23 PM on 6/24/2020

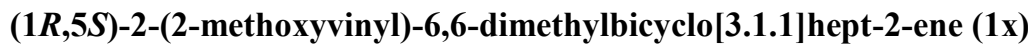

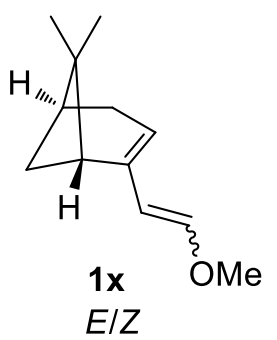

Compound 1x was prepared from (-)-myrtenal in 53\% yield as a colorless oil (474 $\mathrm{mg}$ ). Due to the instability of $\mathbf{1 x}$ under room temperature, it was put into the borylation reaction immediately after purification by column chromatography on silica gel. The NMR spectral data of $\mathbf{1} \mathbf{x}$ were deficient but it could be easily judged from GC-MS that two isomers existed (45:55). EI-MS ( $\mathrm{m} / \mathrm{z}$, relative intensity, $\mathrm{rt}=$ $6.417 \mathrm{~min}): 178\left(\mathrm{M}^{+}, 72\right), 163$ (62), 135(95), 131(65), 122 (32), 110 (21), 105 (80), 103(84), 91 (100), 86 (38), 77(55), 65 (24), 53(21). EI-MS (m/z, relative intensity, $\mathrm{rt}=6.812 \mathrm{~min}): 178\left(\mathrm{M}^{+}, 77\right), 163(56)$, 135(84), 131(64), 117 (30), 110 (22), 105 (79), 103(83), 91 (100), 86 (61), 77(54), 73 (20), 65 (24), 53(20). ATR-FTIR $\left(\mathrm{cm}^{-1}\right): 1422,1263,896,735,703$. HRMS m/z (ESI) calcd for $\mathrm{C}_{12} \mathrm{H}_{19} \mathrm{O}(\mathrm{M}+\mathrm{H})^{+}$ 179.1430, found 179.1429 




\section{General Procedure for Borylation of Alkenyl Methyl Ethers}



To a flame dried $25 \mathrm{~mL}$ Schlenk tube was added Ni(acac) $2(0.05 \mathrm{mmol}, 12.8 \mathrm{mg})$, XantPhos $(0.075$ $\mathrm{mol}, 43.4 \mathrm{mg})$ and $\mathrm{Zn}(0.1 \mathrm{mmol}, 6.5 \mathrm{mg})$, then $\mathrm{B}_{2} \mathrm{Pin}_{2}(1.0 \mathrm{mmol}, 254.0 \mathrm{mg})$ and $\mathrm{K}_{3} \mathrm{PO}_{4}(0.5 \mathrm{mmol}$, $106.1 \mathrm{mg}$ ) were added. The tube was vacuumed and refilled with nitrogen three times followed by the addition of anhydrous toluene $(1.25 \mathrm{~mL})$ and cyclohexane $(3.75 \mathrm{~mL})$. Substrates $1(0.5 \mathrm{mmol})$ was also added with a syringe under nitrogen atmosphere and the plug is screwed. After that, The reaction was stirred under $120^{\circ} \mathrm{C}$ in the heating module for $24 \mathrm{~h}$. Then the mixture was cooled to room temperature, the solvents were removed under reduced pressure and the crude product was purified through flash chromatography with petroleum ether and ethyl actate as the eluent to afford the pure product 2.

\section{(E)-4,4,5,5-tetramethyl-2-styryl-1,3,2-dioxaborolane (2a)}<smiles>Br[B]/C=C/c1ccccc1</smiles>

$2 a$

Eluent: petroleum ether/ethyl acetate (100:1). Colorless oil (93.1 mg, 81\%). ${ }^{1} \mathrm{H}$ NMR (400 MHz, $\left.\mathrm{CDCl}_{3}\right) \delta 7.53-7.46(\mathrm{~m}, 2 \mathrm{H}), 7.40(\mathrm{~d}, J=18.4 \mathrm{~Hz}, 1 \mathrm{H})$, $7.37-7.26(\mathrm{~m}, 3 \mathrm{H}), 6.18(\mathrm{~d}, J=18.4 \mathrm{~Hz}, 1 \mathrm{H}), 1.32(\mathrm{~s}, 12 \mathrm{H}) .{ }^{13} \mathrm{C}$ NMR $(101$ 
$\left.\mathrm{MHz}_{\mathrm{CDCl}}\right) \delta 149.5,137.4,128.9,128.5,127.0,83.3,24.8$. The spectra data are consistent with those in literature report. ${ }^{\mathrm{S} 7}$

\section{(E)-4,4,5,5-tetramethyl-2-(4-methylstyryl)-1,3,2-dioxaborolane (2b)}<smiles>Cc1ccc(/C=C/[Bi]c2ccccc2)cc1</smiles>

Eluent: petroleum ether/ethyl acetate (100:1). Colorless oil (97.6 mg, 80\%). ${ }^{1} \mathrm{H}$ NMR $\left(400 \mathrm{MHz}, \mathrm{CDCl}_{3}\right) \delta 7.44-7.31(\mathrm{~m}, 3 \mathrm{H}), 7.14(\mathrm{~d}, J=7.9$ $\mathrm{Hz}, 2 \mathrm{H}), 6.11(\mathrm{~d}, J=18.4 \mathrm{~Hz}, 1 \mathrm{H}), 2.35(\mathrm{~s}, 3 \mathrm{H}), 1.31(\mathrm{~s}, 12 \mathrm{H}) .{ }^{13} \mathrm{C} \mathrm{NMR}$ $\left(101 \mathrm{MHz}, \mathrm{CDCl}_{3}\right) \delta 149.4,139.0,134.8,129.3,127.0,83.3,24.8,21.3$. The spectra data are consistent with those in literature report. ${ }^{\mathrm{S} 7}$

(E)-4,4,5,5-tetramethyl-2-(3-methylstyryl)-1,3,2-dioxaborolane (2c)<smiles>Cc1cccc(C=C[Bi]c2ccccc2)c1</smiles>

Eluent: petroleum ether/ethyl acetate (100:1). Colorless oil (93.8 mg, 77\%). ${ }^{1} \mathrm{H}$ NMR (400 MHz, $\left.\mathrm{CDCl}_{3}\right) \delta 7.37(\mathrm{~d}, \mathrm{~J}=18.4 \mathrm{~Hz}, 1 \mathrm{H}), 7.33-7.27$ $(\mathrm{m}, 2 \mathrm{H}), 7.23(\mathrm{t}, \mathrm{J}=7.8 \mathrm{~Hz}, 1 \mathrm{H}), 7.11(\mathrm{~d}, \mathrm{~J}=7.5 \mathrm{~Hz}, 1 \mathrm{H}), 6.15(\mathrm{~d}, \mathrm{~J}=18.4$ $\mathrm{Hz}, 1 \mathrm{H}), 2.35$ (s, 3H), 1.31 (s, 12H). ${ }^{13} \mathrm{C} \mathrm{NMR}\left(101 \mathrm{MHz}, \mathrm{CDCl}_{3}\right) \delta 149.6,138.1,137.4,129.7,128.4$, $127.8,124.2,83.3,24.8,21.4$. The spectra data are consistent with those in literature report. ${ }^{\mathrm{S} 8}$

\section{(E)-4,4,5,5-tetramethyl-2-(2-methylstyryl)-1,3,2-dioxaborolane (2d)}<smiles>Cc1ccccc1/C=C/[Bi]Br</smiles>

2d

Eluent: petroleum ether/ethyl acetate (100:1). Colorless oil $(70.7 \mathrm{mg}, 58 \%) .{ }^{1} \mathrm{H}$ NMR (400 MHz, $\left.\mathrm{CDCl}_{3}\right) \delta 7.66(\mathrm{~d}, J=18.3 \mathrm{~Hz}, 1 \mathrm{H}), 7.61-7.51(\mathrm{~m}, 1 \mathrm{H}), 7.23$ $-7.09(\mathrm{~m}, 3 \mathrm{H}), 6.10(\mathrm{~d}, J=18.3 \mathrm{~Hz}, 1 \mathrm{H}), 2.43(\mathrm{~s}, 3 \mathrm{H}), 1.32(\mathrm{~s}, 12 \mathrm{H}) .{ }^{13} \mathrm{C} \mathrm{NMR}$

$\left(101 \mathrm{MHz}, \mathrm{CDCl}_{3}\right) \delta 147.1,136.6,136.2,130.3,128.5,126.0,125.7,83.2,24.8$,

19.8. The spectra data are consistent with those in literature report. ${ }^{\mathrm{S} 7}$

(E)-2-(4-(tert-butyl)styryl)-4,4,5,5-tetramethyl-1,3,2-dioxaborolane (2e)<smiles>CC(C)(C)c1ccc(/C=C/[B]c2ccccc2)cc1</smiles>

$2 e$

Eluent: petroleum ether/ethyl acetate (100:1). White solid (107.1 mg, 75\%). ${ }^{1} \mathrm{H} \mathrm{NMR}\left(400 \mathrm{MHz}, \mathrm{CDCl}_{3}\right) \delta 7.49-7.31(\mathrm{~m}, 5 \mathrm{H}), 6.13(\mathrm{~d}, J=18.4$ $\mathrm{Hz}, 1 \mathrm{H}), 1.32$ (s, 21H). ${ }^{13} \mathrm{C} \mathrm{NMR}\left(101 \mathrm{MHz}, \mathrm{CDCl}_{3}\right) \delta 152.1,149.4,134.7$,

$126.8,125.5,83.2,34.7,31.2,24.8$. The spectra data are consistent with those in literature report. ${ }^{\mathrm{S} 7}$

(E)-2-(4-methoxystyryl)-4,4,5,5-tetramethyl-1,3,2-dioxaborolane (2f) 
<smiles>COc1ccc(/C=C/c2ccccc2)cc1</smiles>

2f

Eluent: petroleum ether/ethyl acetate (80:1). Colorless oil (106.7 mg, 82\%). ${ }^{1} \mathrm{H}$ NMR $\left(400 \mathrm{MHz}, \mathrm{CDCl}_{3}\right) \delta 7.49-7.39$ (m, 2H), 7.35 (d, $J=$ $18.4 \mathrm{~Hz}, 1 \mathrm{H}), 6.92-6.80(\mathrm{~m}, 2 \mathrm{H}), 6.01(\mathrm{~d}, J=18.4 \mathrm{~Hz}, 1 \mathrm{H}), 3.81(\mathrm{~s}, 3 \mathrm{H})$, $1.31(\mathrm{~s}, 12 \mathrm{H}) .{ }^{13} \mathrm{C}$ NMR $\left(101 \mathrm{MHz}, \mathrm{CDCl}_{3}\right) \delta 160.3,149.0,130.4,128.4,113.9,83.2,55.3,24.8$. The spectra data are consistent with those in literature report. ${ }^{\mathrm{S} 8}$

\section{(E)- $N, N$-dimethyl-4-(2-(4,4,5,5-tetramethyl-1,3,2-dioxaborolan-2-yl)vinyl)aniline (2g)}<smiles>CN(C)c1ccc(/C=C/[Bi]c2ccccc2)cc1</smiles>

Eluent: petroleum ether/ethyl acetate (80:1). White solid (120.2 mg, 88\%). ${ }^{1} \mathrm{H}$ NMR (400 MHz, $\left.\mathrm{CDCl}_{3}\right) \delta 7.45-7.37(\mathrm{~m}, 2 \mathrm{H}), 7.34$ (d, $J=$ $18.3 \mathrm{~Hz}, 1 \mathrm{H}), 6.67(\mathrm{~d}, J=8.8 \mathrm{~Hz}, 2 \mathrm{H}), 5.92(\mathrm{~d}, J=18.3 \mathrm{~Hz}, 1 \mathrm{H}), 2.98(\mathrm{~s}$, 6H), $1.31(\mathrm{~s}, 12 \mathrm{H}) .{ }^{13} \mathrm{C}$ NMR $\left(101 \mathrm{MHz}, \mathrm{CDCl}_{3}\right) \delta 150.9,149.8,128.4,125.9,111.9,83.0,40.3,24.8$. The spectra data are consistent with those in literature report. ${ }^{\mathrm{S}}$

\section{(E)-2-(2-([1,1'-biphenyl]-4-yl)vinyl)-4,4,5,5-tetramethyl-1,3,2-dioxaborolane (2h)}<smiles>C(=C/c1ccc(-c2ccccc2)cc1)\c1ccccc1</smiles>

Eluent: petroleum ether/ethyl acetate (100:1). White solid (116.2 mg, 76\%). ${ }^{1} \mathrm{H}$ NMR $\left(400 \mathrm{MHz}, \mathrm{CDCl}_{3}\right) \delta 7.65-7.52(\mathrm{~m}, 6 \mathrm{H}), 7.49-7.38(\mathrm{~m}$, $3 \mathrm{H}), 7.38-7.31(\mathrm{~m}, 1 \mathrm{H}), 6.21(\mathrm{~d}, J=18.4 \mathrm{~Hz}, 1 \mathrm{H}), 1.33(\mathrm{~s}, 12 \mathrm{H}) .{ }^{13} \mathrm{C} \mathrm{NMR}$ $\left(101 \mathrm{MHz}, \mathrm{CDCl}_{3}\right) \delta 149.0,141.6,140.5,136.4,128.8,127.5,127.4,127.2,127.0,83.4,24.8$. The spectra data are consistent with those in literature report. ${ }^{\mathrm{S} 8}$

\section{(E)-4,4,5,5-tetramethyl-2-(4-(methylthio)styryl)-1,3,2-dioxaborolane (2i)}<smiles>CSc1ccc(/C=C/c2ccccc2)cc1</smiles>

2i

Eluent: petroleum ether/ethyl acetate (80:1). Colorless oil (117.3 mg, 85\%). ${ }^{1} \mathrm{H}$ NMR (400 MHz, $\left.\mathrm{CDCl}_{3}\right) \delta 7.44-7.37(\mathrm{~m}, 2 \mathrm{H}), 7.34(\mathrm{~d}, J=$ $18.4 \mathrm{~Hz}, 1 \mathrm{H}), 7.24-7.17$ (m, 2H), $6.11(\mathrm{~d}, J=18.4 \mathrm{~Hz}, 1 \mathrm{H}), 2.48$ (s, 3H), $1.31(\mathrm{~s}, 12 \mathrm{H}) .{ }^{13} \mathrm{C}$ NMR $\left(101 \mathrm{MHz}, \mathrm{CDCl}_{3}\right) \delta 148.8,139.6,134.3,127.4,126.1,83.3,24.8,15.4$. The spectra data are consistent with those in literature report. ${ }^{\mathrm{S} 9}$

\section{(E)-2-(4-fluorostyryl)-4,4,5,5-tetramethyl-1,3,2-dioxaborolane (2j)}<smiles>Fc1ccc(/C=C/[Bi]c2ccccc2)cc1</smiles>

2j

Eluent: petroleum ether/ethyl acetate (100:1). Colorless oil (91.6 mg, 74\%). ${ }^{1} \mathrm{H} \mathrm{NMR}\left(400 \mathrm{MHz}, \mathrm{CDCl}_{3}\right) \delta 7.51-7.39(\mathrm{~m}, 2 \mathrm{H}), 7.35(\mathrm{~d}, J=18.4 \mathrm{~Hz}, 1 \mathrm{H})$, $7.02(\mathrm{t}, J=8.7 \mathrm{~Hz}, 2 \mathrm{H}), 6.07(\mathrm{~d}, J=18.4 \mathrm{~Hz}, 1 \mathrm{H}), 1.31(\mathrm{~s}, 12 \mathrm{H}) .{ }^{13} \mathrm{C} \mathrm{NMR}$ $\left(101 \mathrm{MHz}, \mathrm{CDCl}_{3}\right) \delta 163.1(\mathrm{~d}, J=248.6 \mathrm{~Hz}), 148.1,133.7(\mathrm{~d}, J=3.2 \mathrm{~Hz}), 128.7(\mathrm{~d}, J=8.2 \mathrm{~Hz}), 115.5$ 
$(\mathrm{d}, J=21.7 \mathrm{~Hz}), 83.4,24.8 .{ }^{19} \mathrm{~F}$ NMR $\left(376 \mathrm{MHz}, \mathrm{CDCl}_{3}\right) \delta-112.4$. The spectra data are consistent with those in literature report. ${ }^{\mathrm{S} 8}$

\section{(E)-2-(2-fluorostyryl)-4,4,5,5-tetramethyl-1,3,2-dioxaborolanee (2k)}<smiles>Fc1ccccc1/C=C/[B]c1ccccc1</smiles>

$2 \mathbf{k}$

Eluent: petroleum ether/ethyl acetate (100:1). Colorless oil $(74.5 \mathrm{mg}, 60 \%) .{ }^{1} \mathrm{H}$ NMR (400 MHz, $\left.\mathrm{CDCl}_{3}\right) \delta 7.66-7.51(\mathrm{~m}, 2 \mathrm{H}), 7.36-7.19(\mathrm{~m}, 1 \mathrm{H}), 7.16-7.07$ (m, 1H), $7.03(\mathrm{ddd}, J=10.8,8.2,1.2 \mathrm{~Hz}, 1 \mathrm{H}), 6.24(\mathrm{~d}, J=18.6 \mathrm{~Hz}, 1 \mathrm{H}), 1.31(\mathrm{~s}$, 12H). ${ }^{13} \mathrm{C} \mathrm{NMR}\left(101 \mathrm{MHz}, \mathrm{CDCl}_{3}\right) \delta 160.7(\mathrm{~d}, J=251.6 \mathrm{~Hz}), 141.3(\mathrm{~d}, J=4.1$

Hz), $130.2(\mathrm{~d}, J=8.6 \mathrm{~Hz}), 127.4(\mathrm{~d}, J=3.3 \mathrm{~Hz}), 125.4(\mathrm{~d}, J=11.6 \mathrm{~Hz}), 124.1(\mathrm{~d}, J=3.6 \mathrm{~Hz}), 115.8$ (d, $J=22.1 \mathrm{~Hz}), 83.4,24.8 .{ }^{19} \mathrm{~F} \mathrm{NMR}\left(376 \mathrm{MHz}, \mathrm{CDCl}_{3}\right) \delta-117.7$. The spectra data are consistent with those in literature report. ${ }^{\mathrm{S} 7}$

\section{(E)-4,4,5,5-tetramethyl-2-(4-(trifluoromethyl)styryl)-1,3,2-dioxaborolane (21)}<smiles>FC(F)(F)c1ccc(/C=C/[B]c2ccccc2)cc1</smiles>

21

Eluent: petroleum ether/ethyl acetate (100:1). Colorless oil (104.4 mg, 70\%). ${ }^{1} \mathrm{H}$ NMR (400 MHz, $\left.\mathrm{CDCl}_{3}\right) \delta 7.63-7.52(\mathrm{~m}, 4 \mathrm{H}), 7.40(\mathrm{~d}, J=18.4$

$\mathrm{Hz}, 1 \mathrm{H}), 6.26(\mathrm{~d}, J=18.4 \mathrm{~Hz}, 1 \mathrm{H}), 1.32$ (s, 12H). ${ }^{13} \mathrm{C} \mathrm{NMR}(101 \mathrm{MHz}$, $\left.\mathrm{CDCl}_{3}\right) \delta 147.7,140.7,130.4(\mathrm{q}, J=32.4 \mathrm{~Hz}), 128.3,127.1,125.5(\mathrm{q}, J=3.9 \mathrm{~Hz}), 124.1(\mathrm{q}, J=272.0$ $\mathrm{Hz}), 83.6,24.8 .{ }^{19} \mathrm{~F} \mathrm{NMR}\left(376 \mathrm{MHz}, \mathrm{CDCl}_{3}\right) \delta-62.6$. The spectra data are consistent with those in literature report. ${ }^{\mathrm{S} 8}$

(E)-methyl 4-(2-(4,4,5,5-tetramethyl-1,3,2-dioxaborolan-2-yl)vinyl)benzoate $(2 \mathrm{~m})^{[6]}$<smiles>COC(=O)c1ccc(/C=C/[B]c2ccccc2)cc1</smiles>

$2 m$

Eluent: petroleum ether/ethyl acetate (60:1). White solid (97.8 mg, 68\%). ${ }^{1} \mathrm{H} \mathrm{NMR}\left(400 \mathrm{MHz}, \mathrm{CDCl}_{3}\right) \delta 7.99(\mathrm{~d}, J=8.4 \mathrm{~Hz}, 2 \mathrm{H}), 7.58-$ $7.46(\mathrm{~m}, 2 \mathrm{H}), 7.40(\mathrm{~d}, J=18.4 \mathrm{~Hz}, 1 \mathrm{H}), 6.26(\mathrm{~d}, J=18.4 \mathrm{~Hz}, 1 \mathrm{H}), 3.90$

(s, 3H), $1.30(\mathrm{~s}, 12 \mathrm{H}) .{ }^{13} \mathrm{C} \mathrm{NMR}\left(101 \mathrm{MHz}, \mathrm{CDCl}_{3}\right) \delta 166.7,148.1,141.6,130.1,129.9,126.8,83.5$, 52.1, 24.8. The spectra data are consistent with those in literature report. S10 $^{\text {S }}$

\section{(E)-N,N-diethyl-4-(2-(4,4,5,5-tetramethyl-1,3,2-dioxaborolan-2-yl)vinyl)benzamide (2n)}<smiles>CCN(CC)C(=O)c1ccc(/C=C/[Pb]c2ccccc2)cc1</smiles>
Eluent: petroleum ether/ethyl acetate (10:1). White solid (125.0 mg, 76\%). ${ }^{1} \mathrm{H}$ NMR (400 MHz, $\left.\mathrm{CDCl}_{3}\right) \delta 7.54-7.45(\mathrm{~m}, 2 \mathrm{H}), 7.43-7.29$ (m, 3H), $6.19(\mathrm{~d}, J=18.5 \mathrm{~Hz}, 1 \mathrm{H}), 3.73-3.70(\mathrm{~m}, 4 \mathrm{H}), 1.31(\mathrm{~s}, 12 \mathrm{H})$,

$1.30-1.00(\mathrm{~m}, 6 \mathrm{H}) .{ }^{13} \mathrm{C} \mathrm{NMR}\left(101 \mathrm{MHz}, \mathrm{CDCl}_{3}\right) \delta 170.9,148.5,138.2,137.5,127.0,126.7,83.4,43.2$, 
39.2, 24.8, 14.2, 12.9. ATR-FTIR $\left(\mathrm{cm}^{-1}\right): 1421,1268,892,734,702$. HRMS m/z (ESI) calcd for $\mathrm{C}_{19} \mathrm{H}_{29} \mathrm{BNO}_{3}(\mathrm{M}+\mathrm{H})^{+} 330.2235$, found 330.2233 .

\section{(E)-2-(3,5-dimethylstyryl)-4,4,5,5-tetramethyl-1,3,2-dioxaborolane (2o)}<smiles>Cc1cc(C)cc(C=C[B]c2ccccc2)c1</smiles>

Eluent: petroleum ether/ethyl acetate (100:1). Colorless oil (81.3 mg, 63\%). ${ }^{1} \mathrm{H}$ NMR $\left(400 \mathrm{MHz}, \mathrm{CDCl}_{3}\right) \delta 7.34(\mathrm{~d}, J=18.4 \mathrm{~Hz}, 1 \mathrm{H}), 7.11$ (brs, 2H), 6.94 (brs, 1H), $6.13(\mathrm{~d}, J=18.4 \mathrm{~Hz}, 1 \mathrm{H}), 2.31(\mathrm{~s}, 6 \mathrm{H}), 1.31(\mathrm{~s}, 12 \mathrm{H})$.

${ }^{13}$ C NMR (101 MHz, CDCl $) \delta 149.8,137.9,137.4,130.6,124.9,83.2,24.8,21.2$. ATR-FTIR $\left(\mathrm{cm}^{-1}\right)$ : 1264, 907, 729, 706, 650. HRMS m/z (ESI) calcd for $\mathrm{C}_{16} \mathrm{H}_{24} \mathrm{BO}_{2}(\mathrm{M}+\mathrm{H})^{+}$259.1864, found 259.1864.

\section{(E)-2-(3,5-dimethoxystyryl)-4,4,5,5-tetramethyl-1,3,2-dioxaborolane (2p)}<smiles>COc1cc(/C=C/[Bi]c2ccccc2)cc(OC)c1</smiles>

Eluent: petroleum ether/ethyl acetate (50:1). White solid $(92.6 \mathrm{mg}$, 64\%). ${ }^{1} \mathrm{H} \mathrm{NMR}\left(400 \mathrm{MHz}, \mathrm{CDCl}_{3}\right) \delta 7.32(\mathrm{~d}, J=18.4 \mathrm{~Hz}, 1 \mathrm{H}), 6.65(\mathrm{~d}, J$ $=2.2 \mathrm{~Hz}, 2 \mathrm{H}), 6.42(\mathrm{t}, J=2.3 \mathrm{~Hz}, 1 \mathrm{H}), 6.14(\mathrm{~d}, J=18.4 \mathrm{~Hz}, 1 \mathrm{H}), 3.79(\mathrm{~s}$, 6H), $1.31(\mathrm{~s}, 12 \mathrm{H}) .{ }^{13} \mathrm{C}$ NMR $\left(101 \mathrm{MHz}, \mathrm{CDCl}_{3}\right) \delta 160.8,149.3,139.5$,

$104.9,101.3,83.4,55.3,24.8$. The spectra data are consistent with those in literature report. ${ }^{\mathrm{s} 11}$

(E)-2-(2-(2,3-dihydrobenzofuran-5-yl)vinyl)-4,4,5,5-tetramethyl-1,3,2-dioxaborolane (2q)

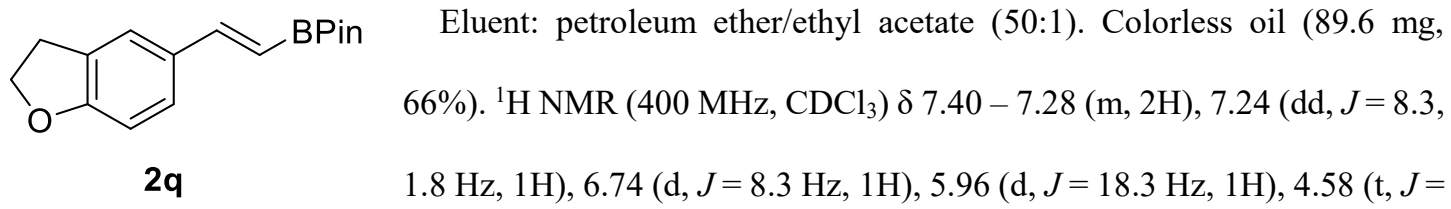
$8.7 \mathrm{~Hz}, 2 \mathrm{H}), 3.20(\mathrm{t}, J=8.7 \mathrm{~Hz}, 2 \mathrm{H}), 1.30(\mathrm{~s}, 12 \mathrm{H}) .{ }^{13} \mathrm{C} \mathrm{NMR}\left(101 \mathrm{MHz}, \mathrm{CDCl}_{3}\right) \delta 161.0,149.5,130.5$, 128.1, 127.5, 123.2, 109.2, 83.2, 71.6, 29.4, 24.8. ATR-FTIR $\left(\mathrm{cm}^{-1}\right): 1423,1261,895,733,705$. HRMS $\mathrm{m} / \mathrm{z}(\mathrm{ESI})$ calcd for $\mathrm{C}_{16} \mathrm{H}_{22} \mathrm{BO}_{3}(\mathrm{M}+\mathrm{H})^{+} 273.1657$, found 273.1653 .

\section{(E)-4,4,5,5-tetramethyl-2-(2-(naphthalen-2-yl)vinyl)-1,3,2-dioxaborolane (2r)}



Eluent: petroleum ether/ethyl acetate (100:1). Yellowish solid (110.8 mg, 79\%). ${ }^{1} \mathrm{H} \mathrm{NMR}\left(400 \mathrm{MHz}, \mathrm{CDCl}_{3}\right) \delta 7.88-7.76(\mathrm{~m}, 4 \mathrm{H}), 7.70(\mathrm{dd}, J=8.6$, $1.7 \mathrm{~Hz}, 1 \mathrm{H}), 7.57(\mathrm{~d}, J=18.4 \mathrm{~Hz}, 1 \mathrm{H}), 7.51-7.42(\mathrm{~m}, 2 \mathrm{H}), 6.29(\mathrm{~d}, J=$

$18.4 \mathrm{~Hz}, 1 \mathrm{H}), 1.34(\mathrm{~s}, 12 \mathrm{H}) .{ }^{13} \mathrm{C} \mathrm{NMR}\left(101 \mathrm{MHz}, \mathrm{CDCl}_{3}\right) \delta 149.5,134.9,133.7,133.4,128.4,128.2$, $128.0,127.7,126.4,126.3,123.4,83.4,24.8$. The spectra data are consistent with those in literature report. $^{\text {S7 }}$ 


\section{(E)-4,4,5,5-tetramethyl-2-(2-phenylprop-1-en-1-yl)-1,3,2-dioxaborolane (2s)}<smiles>CC(=C[Pb]c1ccccc1)c1ccccc1</smiles>

2s

Eluent: petroleum ether/ethyl acetate (100:1). Colorless oil (57.0 mg, 47\%). ${ }^{1} \mathrm{H}$ $\operatorname{NMR}\left(400 \mathrm{MHz}, \mathrm{CDCl}_{3}\right) \delta 7.54-7.46(\mathrm{~m}, 2 \mathrm{H}), 7.37-7.26(\mathrm{~m}, 3 \mathrm{H}), 5.75(\mathrm{~s}, 1 \mathrm{H})$, $2.41(\mathrm{~s}, 3 \mathrm{H}), 1.32(\mathrm{~s}, 12 \mathrm{H}) .{ }^{13} \mathrm{C}$ NMR $\left(101 \mathrm{MHz}, \mathrm{CDCl}_{3}\right) \delta 157.8,143.8,128.1$, 127.9, 125.8, 82.9, 24.9, 20.1. The spectra data are consistent with those in literature report. ${ }^{\mathrm{S}}{ }^{7}$

\section{(E)-9-ethyl-3-(1-(4,4,5,5-tetramethyl-1,3,2-dioxaborolan-2-yl)prop-1-en-2-yl)-9H-carbazole} $(2 \mathrm{t})$<smiles>CCn1c2ccccc2c2cc(/C=C/[Pb]c3ccccc3)ccc21</smiles>

Eluent: petroleum ether/ethyl acetate (80:1). Yellowish solid (140.3 mg, 81\%). ${ }^{1} \mathrm{H}$ NMR $\left(400 \mathrm{MHz}, \mathrm{CDCl}_{3}\right) \delta 8.23(\mathrm{~d}, J=1.7 \mathrm{~Hz}, 1 \mathrm{H}), 8.09$ (dt, $J=7.8,1.0 \mathrm{~Hz}, 1 \mathrm{H}), 7.73-7.58(\mathrm{~m}, 2 \mathrm{H}), 7.48(\mathrm{ddd}, J=8.2,7.0$, $1.2 \mathrm{~Hz}, 1 \mathrm{H}), 7.43-7.32(\mathrm{~m}, 2 \mathrm{H}), 7.29-7.20(\mathrm{~m}, 1 \mathrm{H}), 6.20(\mathrm{~d}, J=18.4 \mathrm{~Hz}, 1 \mathrm{H}), 4.35$ (q, $J=7.2 \mathrm{~Hz}$, 2H), $1.43(\mathrm{t}, J=7.2 \mathrm{~Hz}, 3 \mathrm{H}), 1.35(\mathrm{~s}, 12 \mathrm{H}) .{ }^{13} \mathrm{C}$ NMR $\left(101 \mathrm{MHz}, \mathrm{CDCl}_{3}\right) \delta 150.6,140.5,140.3,128.8$, 125.8, 124.8, 123.0, 123.0, 120.4, 119.8, 119.1, 108.6, 108.5, 83.1, 37.6, 24.8, 13.8. ATR-FTIR $\left(\mathrm{cm}^{-1}\right)$ : $2977,1472,1455,1332,1233,1146,1124,745,728,670,630$. HRMS m/z (ESI) calcd for $\mathrm{C}_{22} \mathrm{H}_{27} \mathrm{BNO}_{2}$ $(\mathrm{M}+\mathrm{H})^{+} 348.2129$, found 348.2122 .

\section{H-benzo[e][1,2]oxaborinin-2-ol (2u)}<smiles>OB1C=Cc2ccccc2O1</smiles>

$2 \mathrm{u}$

Eluent: petroleum ether/ethyl acetate (40:1). White solid $(44.7 \mathrm{mg}, 61 \%) .{ }^{1} \mathrm{H}$ NMR $\left(400 \mathrm{MHz}, \mathrm{CDCl}_{3}\right) \delta 7.78(\mathrm{~d}, J=11.9 \mathrm{~Hz}, 1 \mathrm{H}), 7.47-7.31(\mathrm{~m}, 2 \mathrm{H}), 7.28-$ $7.23(\mathrm{~m}, 1 \mathrm{H}), 7.15(\mathrm{td}, J=7.4,1.2 \mathrm{~Hz}, 1 \mathrm{H}), 6.22(\mathrm{~d}, J=11.8 \mathrm{~Hz}, 1 \mathrm{H}), 4.54$ (brs, 1H). ${ }^{13} \mathrm{C}$ NMR $\left(101 \mathrm{MHz}, \mathrm{CDCl}_{3}\right) \delta 152.3,149.4,129.4,128.7,124.4,122.3,118.4$. The spectra data was consistent with literature report. ${ }^{\text {S12 }}$

\section{(E)-2-(2-(cyclohex-1-en-1-yl)vinyl)-4,4,5,5-tetramethyl-1,3,2-dioxaborolane (2v)}

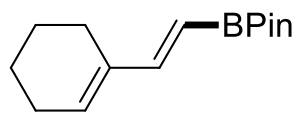

$2 \mathbf{v}$

Eluent: petroleum ether/ethyl acetate (80:1). Colorless oil $(65.6 \mathrm{mg}, 56 \%) .{ }^{1} \mathrm{H}$ NMR (400 MHz, $\left.\mathrm{CDCl}_{3}\right) \delta 7.02(\mathrm{~d}, J=18.2 \mathrm{~Hz}, 1 \mathrm{H}), 5.96(\mathrm{t}, J=3.9 \mathrm{~Hz}, 1 \mathrm{H})$, $5.42(\mathrm{~d}, J=18.2 \mathrm{~Hz}, 1 \mathrm{H}), 2.22-2.07(\mathrm{~m}, 4 \mathrm{H}), 1.76-1.47(\mathrm{~m}, 4 \mathrm{H}), 1.27(\mathrm{~s}, 12 \mathrm{H})$ ${ }^{13} \mathrm{C}$ NMR $\left(101 \mathrm{MHz}, \mathrm{CDCl}_{3}\right) \delta 153.2,137.1,134.3,83.0,26.2,24.8,23.7,22.4,22.3$. The spectra data was consistent with literature report. ${ }^{\mathrm{S} 13}$ ATR-FTIR $\left(\mathrm{cm}^{-1}\right): 1424,1264,893,735,703 . \mathrm{HRMS} \mathrm{m} / \mathrm{z}$ (ESI) calcd for $\mathrm{C}_{14} \mathrm{H}_{24} \mathrm{BO}_{2}(\mathrm{M}+\mathrm{H})^{+}$235.1864, found 235.1859 . 
$(S, E)-4,4,5,5-t e t r a m e t h y l-2-(2-(4-(p r o p-1-e n-2-y l) c y c l o h e x-1-e n-1-y l) v i n y l)-1,3,2-$ dioxaborolane (2w)

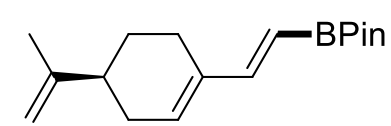

$2 w$
Eluent: petroleum ether/ethyl acetate (80:1). Colorless oil (69.7 mg, 51\%). ${ }^{1} \mathrm{H}$ NMR $\left(400 \mathrm{MHz}, \mathrm{CDCl}_{3}\right) \delta 7.04(\mathrm{~d}, J=18.3 \mathrm{~Hz}, 1 \mathrm{H}), 6.01-5.95$

$(\mathrm{m}, 1 \mathrm{H}), 5.43(\mathrm{~d}, J=18.3 \mathrm{~Hz}, 1 \mathrm{H}), 4.76-4.68(\mathrm{~m}, 2 \mathrm{H}), 2.40-2.22(\mathrm{~m}$,

2H), $2.22-2.01(\mathrm{~m}, 3 \mathrm{H}), 1.74(\mathrm{~s}, 3 \mathrm{H}), 1.66-1.55(\mathrm{~m}, 2 \mathrm{H}), 1.28(\mathrm{~s}, 12 \mathrm{H}) .{ }^{13} \mathrm{C} \mathrm{NMR}\left(101 \mathrm{MHz}, \mathrm{CDCl}_{3}\right)$ $\delta 152.6,149.5,136.8,133.5,108.8,83.1,41.0,31.5,27.3,24.8,24.1,20.8$. The spectra data was consistent with literature report. ${ }^{\mathrm{S} 13} \mathrm{HRMS} \mathrm{m} / \mathrm{z}(\mathrm{ESI})$ calcd for $\mathrm{C}_{17} \mathrm{H}_{28} \mathrm{BO}_{2}(\mathrm{M}+\mathrm{H})^{+} 275.2177$, found 275.2174 .

2-((E)-2-((1R,5S)-6,6-dimethylbicyclo[3.1.1]hept-2-en-2-yl)vinyl)-4,4,5,5-tetramethyl-1,3,2dioxaborolane $(2 x)$

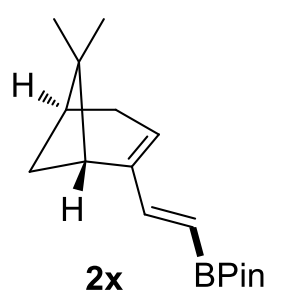

Eluent: petroleum ether/ethyl acetate (80:1). Colorless oil $(57.6 \mathrm{mg}, 42 \%) .{ }^{1} \mathrm{H}$ NMR (400 MHz, $\left.\mathrm{CDCl}_{3}\right) \delta 7.04(\mathrm{~d}, \mathrm{~J}=18.2 \mathrm{~Hz}, 1 \mathrm{H}), 5.82-5.75(\mathrm{~m}, 1 \mathrm{H}), 5.41(\mathrm{~d}$, $\mathrm{J}=18.2 \mathrm{~Hz}, 1 \mathrm{H}), 2.63(\mathrm{td}, \mathrm{J}=5.7,1.5 \mathrm{~Hz}, 1 \mathrm{H}), 2.46-2.27(\mathrm{~m}, 3 \mathrm{H}), 2.15-2.07$ $(\mathrm{m}, 1 \mathrm{H}), 1.30(\mathrm{~s}, 3 \mathrm{H}), 1.27(\mathrm{~s}, 12 \mathrm{H}), 1.09(\mathrm{~d}, \mathrm{~J}=8.8 \mathrm{~Hz}, 1 \mathrm{H}), 0.75(\mathrm{~s}, 3 \mathrm{H}) .{ }^{13} \mathrm{C}$ NMR (101 MHz, $\left.\mathrm{CDCl}_{3}\right) \delta 150.4,147.9,129.2,83.0,40.9,40.2,37.7,32.2,31.2$, 26.3, 24.8, 24.7, 20.8. ATR-FTIR $\left(\mathrm{cm}^{-1}\right): 1424,1264,892,732,701$. HRMS m/z (ESI) calcd for $\mathrm{C}_{17} \mathrm{H}_{28} \mathrm{BO}_{2}(\mathrm{M}+\mathrm{H})^{+}$275.2177, found 275.2172.

\section{Experimental Procedure for Gram-Scale Borylation of 1a}



To a flame dried $100 \mathrm{~mL}$ Schlenk tube was added Ni(acac) 2 (0.6 mmol, $154.2 \mathrm{mg})$, XantPhos $(0.9$ $\mathrm{mmol}, 520.7 \mathrm{mg})$ and $\mathrm{Zn}(1.2 \mathrm{mmol}, 78 \mathrm{mg})$, then $\mathrm{B}_{2} \mathrm{Pin}_{2}(12 \mathrm{mmol}, 3.048 \mathrm{~g})$ and $\mathrm{K}_{3} \mathrm{PO}_{4}(6 \mathrm{mmol}, 1.272$ g) were added. The tube was vacuumed and refilled with nitrogen three times followed by the addition of anhydrous toluene $(15 \mathrm{~mL})$ and cyclohexane $(45 \mathrm{~mL})$. Substrates $\mathbf{1 a}(6 \mathrm{mmol}, 804 \mathrm{mg})$ was also added with a syringe under nitrogen atmosphere and the plug is screwed. After that, The reaction was stirred under $120{ }^{\circ} \mathrm{C}$ in the heating module for $24 \mathrm{~h}$. Then the mixture was cooled to room temperature, the solvents were removed under reduced pressure and the crude product was purified through flash chromatography with petroleum ether and ethyl actate as the eluent to afford the pure product $\mathbf{2 a}$ as a colorless oil in $80 \%$ yield $(1.10 \mathrm{~g})$. 


\section{Experimental Procedure for the Synthesis of Piceatannol (7)}
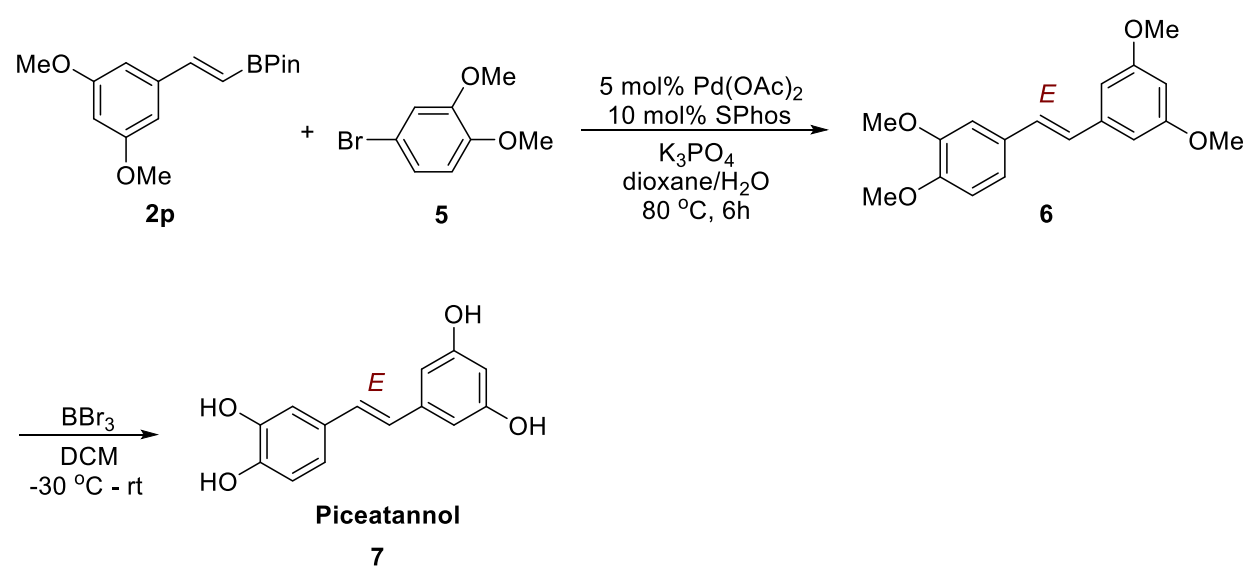

To a flame dried $25 \mathrm{~mL}$ Schlenk tube was added $\mathbf{2 p}(0.3 \mathrm{mmol}, 87.1 \mathrm{mg}), \mathrm{Pd}(\mathrm{OAc})_{2}(0.015 \mathrm{mmol}, 3.4$ $\mathrm{mg})$, SPhos (0.03 mmol, $12.3 \mathrm{mg})$ and $\mathrm{K}_{3} \mathrm{PO}_{4}(0.9 \mathrm{mmol}, 190.8 \mathrm{mg})$. The tube was vacuumed and refilled with nitrogen three times followed by the addition of 1,4-dioxane $(3.0 \mathrm{~mL})$ and $\mathrm{H}_{2} \mathrm{O}(30 \mu \mathrm{L})$. Aryl bromide 5 ( $0.36 \mathrm{mmol}, 78.1 \mathrm{mg})$ was also added with a syringe under nitrogen atmosphere and the plug was screwed. After that, the reaction was stirred under $80{ }^{\circ} \mathrm{C}$ in the heating module for $6 \mathrm{~h}$. Then the mixture was cooled to room temperature, the solvents were removed under reduced pressure and the crude product was purified by flash chromatography with petroleum ether and ethyl actate as the eluent to afford the pure product 6 (white solid, $75.4 \mathrm{mg}, 84 \%) .{ }^{1} \mathrm{H} \mathrm{NMR}\left(400 \mathrm{MHz}, \mathrm{CDCl}_{3}\right) \delta 7.10-6.98(\mathrm{~m}$, $3 \mathrm{H}), 6.95-6.80(\mathrm{~m}, 2 \mathrm{H}), 6.66(\mathrm{~d}, J=2.3 \mathrm{~Hz}, 2 \mathrm{H}), 6.39(\mathrm{t}, J=2.3 \mathrm{~Hz}, 1 \mathrm{H}), 3.94(\mathrm{~s}, 3 \mathrm{H}), 3.90(\mathrm{~s}, 3 \mathrm{H})$, $3.83(\mathrm{~s}, 6 \mathrm{H}) .{ }^{13} \mathrm{C} \mathrm{NMR}\left(101 \mathrm{MHz}, \mathrm{CDCl}_{3}\right) \delta 160.9,149.0,148.9,139.5,130.1,128.9,126.7,119.9,111.1$, 108.7, 104.2, 99.6, 55.8, 55.8, 55.3. The spectra data was consistent with literature report. ${ }^{\mathrm{S} 14}$

To the above compound $6(0.1 \mathrm{mmol}, 30.0 \mathrm{mg})$ in $25 \mathrm{~mL}$ flame dried Schlenk tube was added anhydrous $\mathrm{CH}_{2} \mathrm{Cl}_{2}(1.0 \mathrm{~mL})$. The solution was stirred under $-30^{\circ} \mathrm{C}$ and $\mathrm{BBr}_{3}(1.0 \mathrm{M}$ in $\mathrm{DCM}, 1.0 \mathrm{mmol}$, $1 \mathrm{~mL}$ ) was added dropwise to the mixture. Then the reaction was slowly warmed to room temperature and stirred for another $4 \mathrm{~h}$. When the reaction was finished, $5 \mathrm{~mL}$ of water was added and stirred for another $5 \mathrm{~min}$. The aqueous phase was extracted by DCM and the combined organic phase was dried over anhydrous sodium sulfate. The mixture was then filtered and the solvent was removed under reduced pressure. Further purification by flash chromatography would afford the complete $E$-isomer of Piceatannol (7) as a light brown solid (26.9 mg) in $90 \%$ yield. ${ }^{1} \mathrm{H}$ NMR (400 MHz, $\left.\mathrm{CD}_{3} \mathrm{OD}\right) \delta 6.97(\mathrm{~d}, J$ $=2.0 \mathrm{~Hz}, 1 \mathrm{H}), 6.89(\mathrm{~d}, J=16.3 \mathrm{~Hz}, 1 \mathrm{H}), 6.83(\mathrm{dd}, J=8.2,2.1 \mathrm{~Hz}, 1 \mathrm{H}), 6.78-6.70(\mathrm{~m}, 2 \mathrm{H}), 6.44(\mathrm{~d}, J$ $=2.2 \mathrm{~Hz}, 2 \mathrm{H}), 6.16(\mathrm{t}, J=2.1 \mathrm{~Hz}, 1 \mathrm{H}) .{ }^{13} \mathrm{C} \mathrm{NMR}\left(101 \mathrm{MHz}, \mathrm{CD}_{3} \mathrm{OD}\right) \delta 159.6,146.5,146.5,141.3,131.1$, 
$129.7,127.0,120.2,116.4,113.8,105.8,102.6$. The spectra data was consistent with literature report. ${ }^{\mathrm{S} 15}$ HRMS m/z (ESI) calcd for $\mathrm{C}_{14} \mathrm{H}_{12} \mathrm{NaO}_{4}(\mathrm{M}+\mathrm{Na})^{+} 267.0628$, found 267.0624.

\section{References}

(S1) Hostier, T.; Neouchy, Z.; Ferey, V.; Pardo, D. G.; Cossy,J. Org. Lett. 2018, 20, 1815.

(S2) Al-Smadi, D.; Enugala, T. R.; Norberg, T.; Kihlberg, J.; Widersten, M. Synlett 2018, $29,1187$.

(S3) Gemma, S.; Kunjir, S. and et. al. J. Med. Chem. 2011, 54, 5949.

(S4) Shigeno, M.; Nakamura, R.; Hayashi, K.; Nozawa-Kumada, K.; Kondo, Y. Org. Lett. 2019, $21,6695$.

(S5) Gualandi, A.; Canestrari, P.; Emer, E.; Cozzi, P. G. Adv. Synth. Catal. 2014, 356, 528.

(S6) Su, Y.-L.; Li, L.-L.; Zhou, X.-L.; Dai, Z.-Y.; Wang, P.-S.; Gong, L.-Z. Org. Lett. 2018, 20, 2403.

(S7) Shi, X.; Li, S.; Wu, L. Angew. Chem. Int. Ed. 2019, 58, 16167.

(S8) Yoshii, D.; Jin, X.; Mizuno, N.; Yamaguchi, K. ACS Catal. 2019, 9, 3011.

(S9) Wen, H.; Zhang, L.; Zhu, S.; Liu, G.; Huang, Z. ACS Catal. 2017, 7, 6419.

(S10) Molloy, J. J.; Seath, C. P.; West, M. J.; McLaughlin, C.; Fazakerley, N. J.; Kennedy, A. R.; Nelson, D. J.; Watson, A. J. B. J. Am. Chem. Soc. 2018, 140, 126.

(S11) Zhang, G.; Wu, J.; Zeng, H.; Neary, M. C.; Devany, M.; Zheng, S.; Dub, P. A. ACS Catal. 2019, 9, 874.

(S12) Saito, H.; Otsuka, S.; Nogi, K.; Yorimitsu, H. J. Am. Chem. Soc. 2016, 138, 15315.

(S13) Francois, B.; Eberlin, L.; Berrée, F.; Whiting, A.; Carboni, B. J. Org. Chem. 2020, 85, 5173.

(S14) Chen, G.-G.; Wei, J.-Q.; Yang, X.-L.; Yao, Z.-J. Org. Lett. 2016, 18, 1502.

(S15) Wan, X.; Wang, X.-B.; Yang, M.-H.; Wang, J.-S.; Kong, L.-Y. Bioorg. Med. Chem. 2011, $19,5085$. 


\section{Spectroscopic Data}
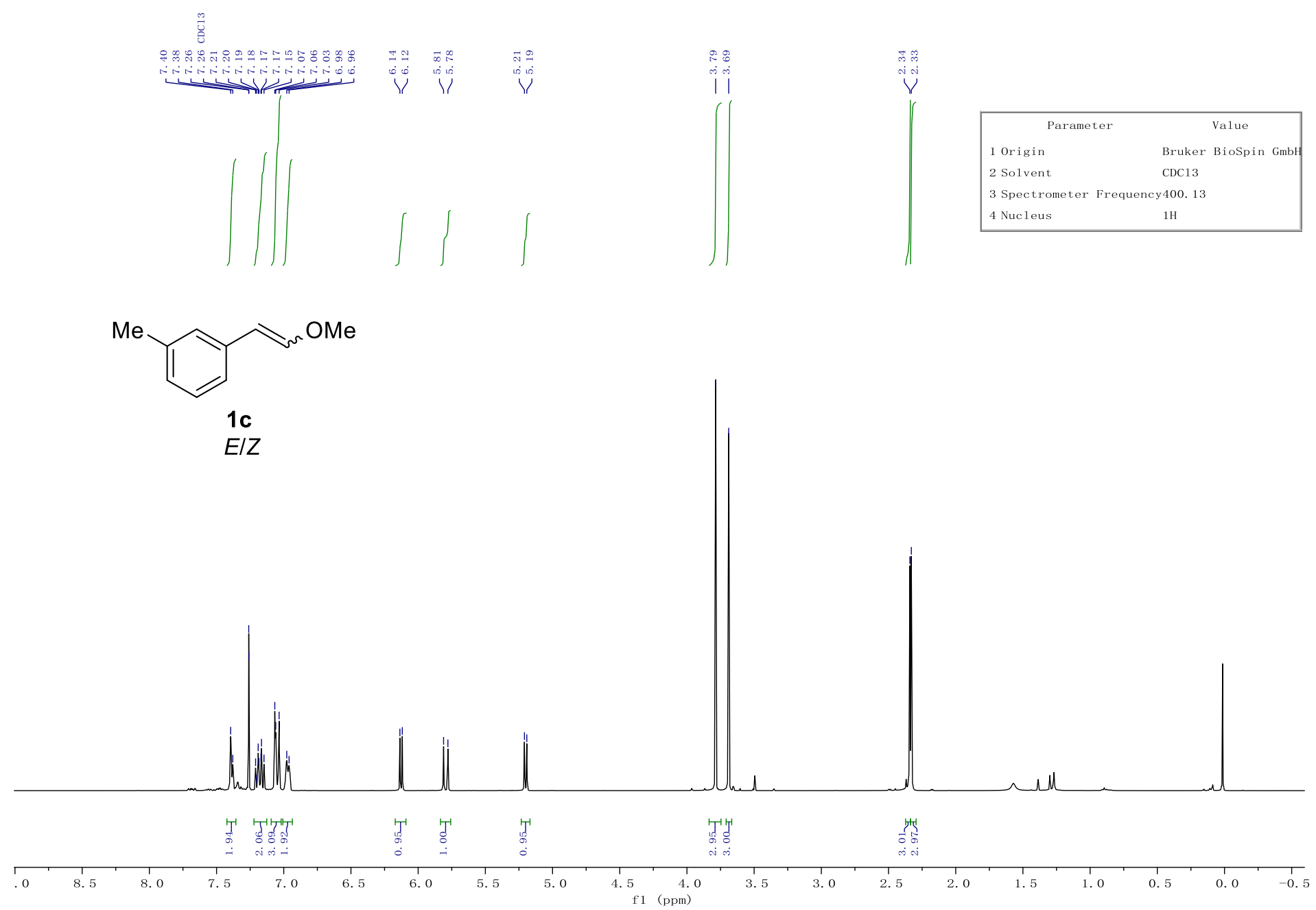


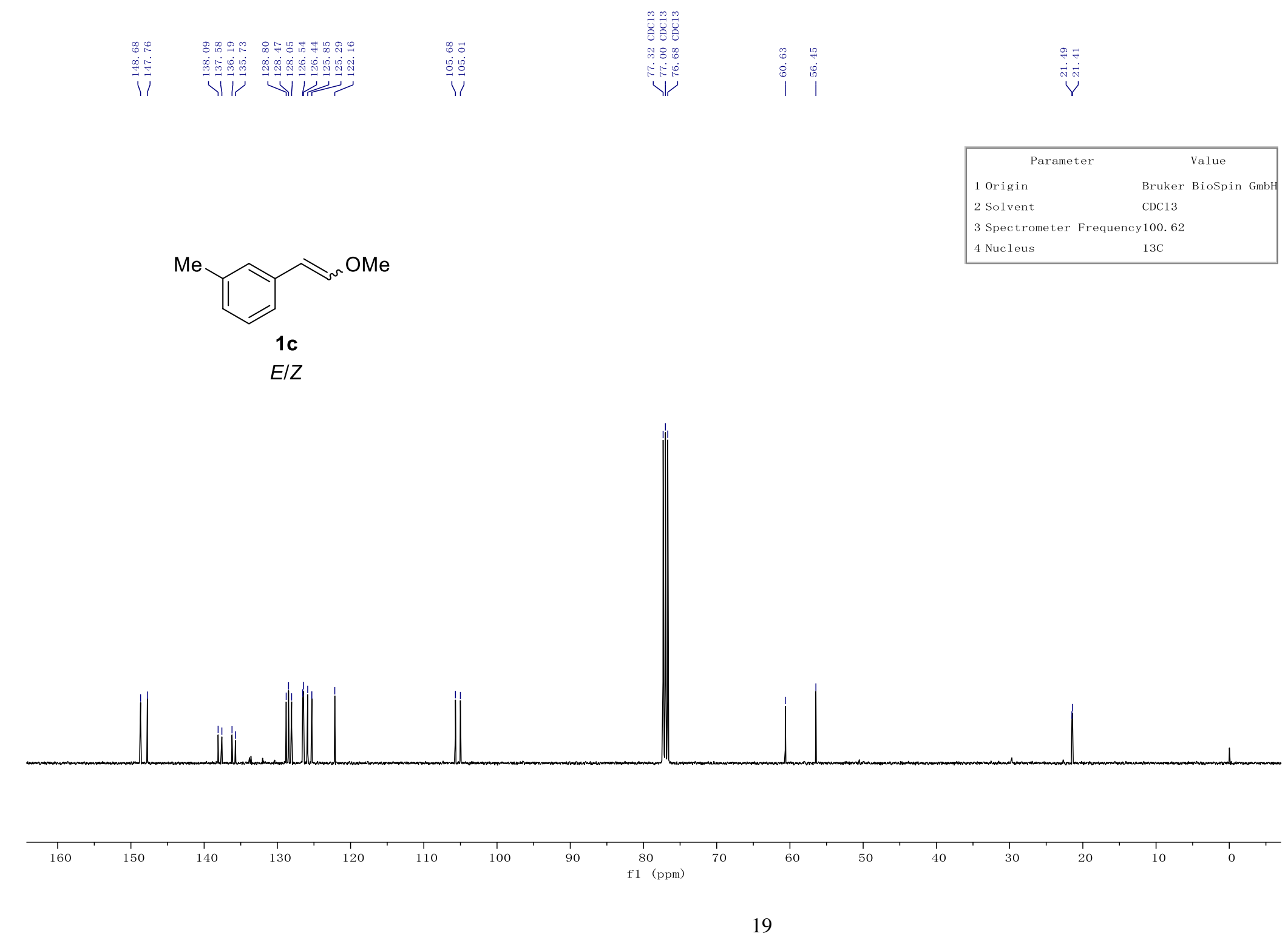




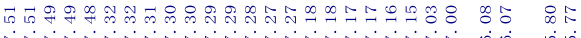

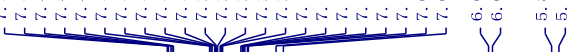

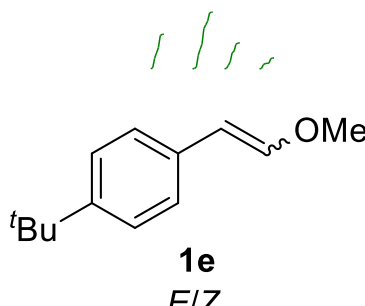

$\iint$

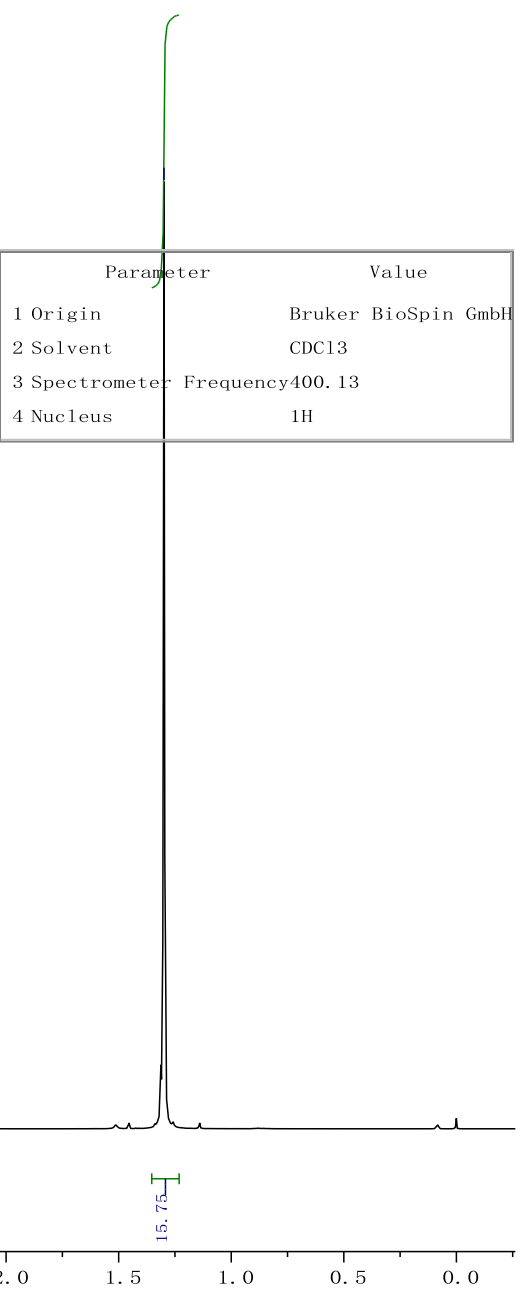



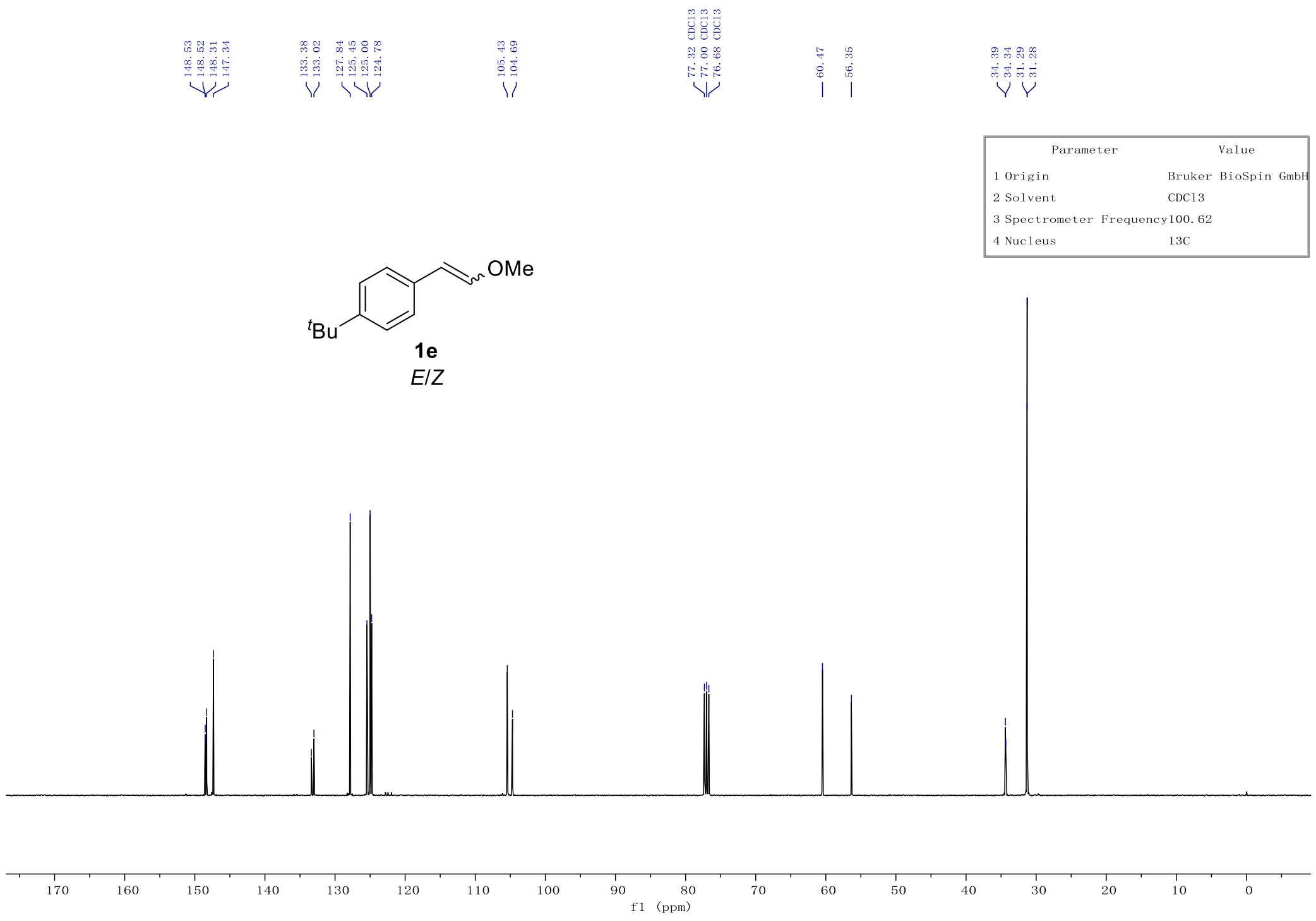


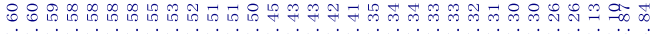

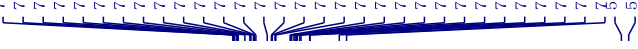

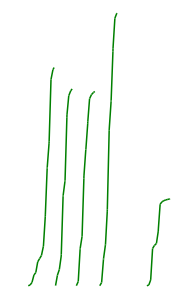

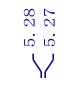

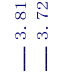

1h

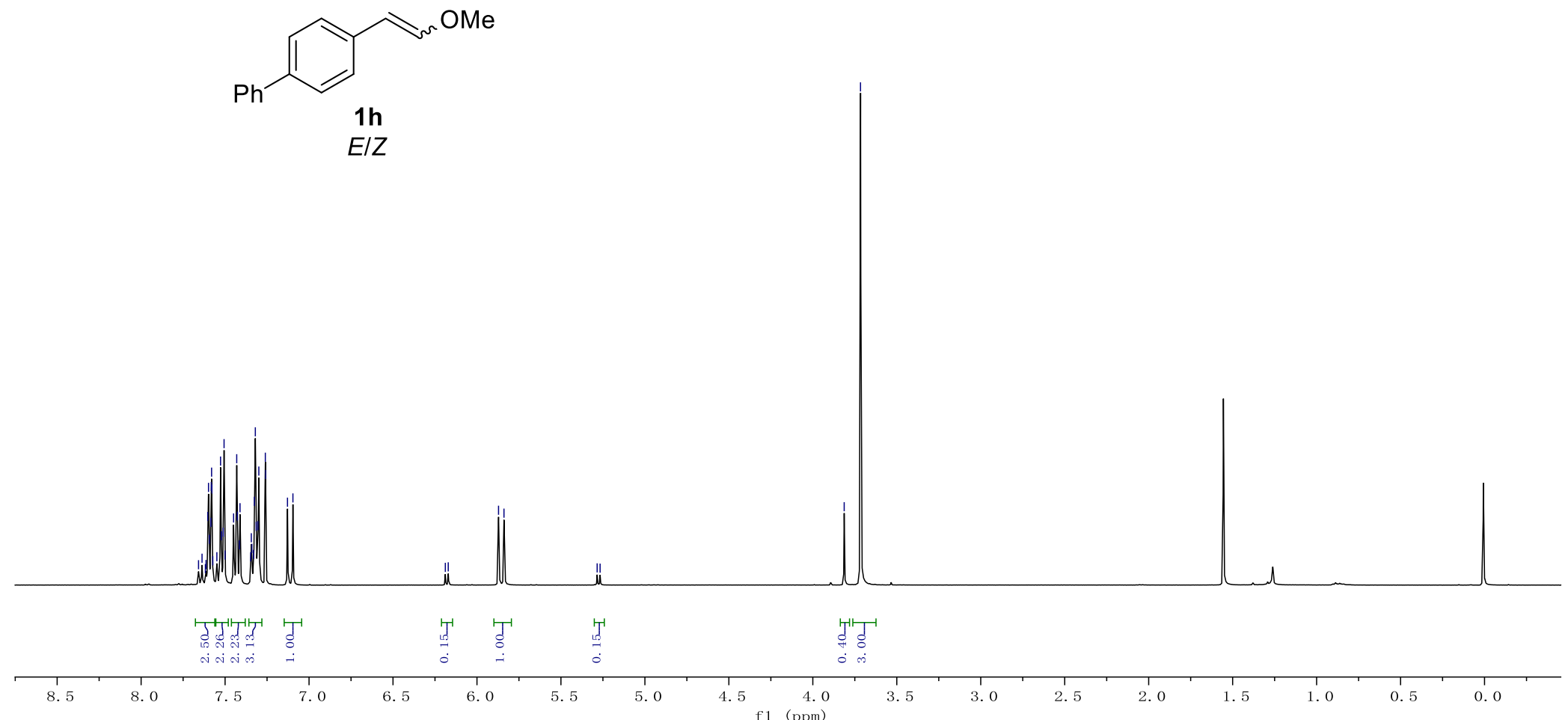



$\int$ 

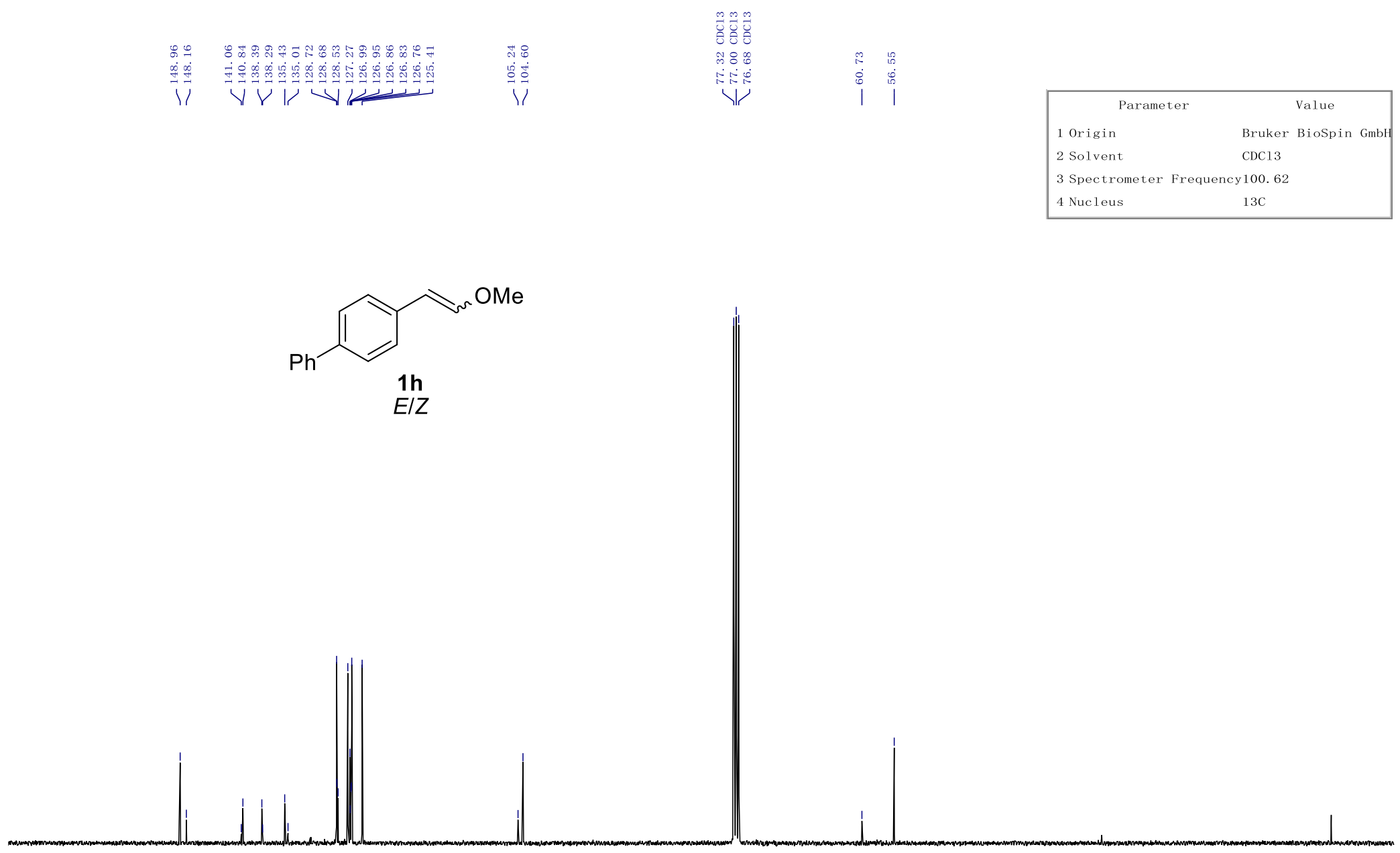

$\frac{1}{170}$

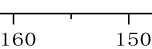

140
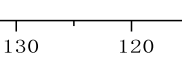

80
$\mathrm{f} 1 \quad(\mathrm{ppm})$ 


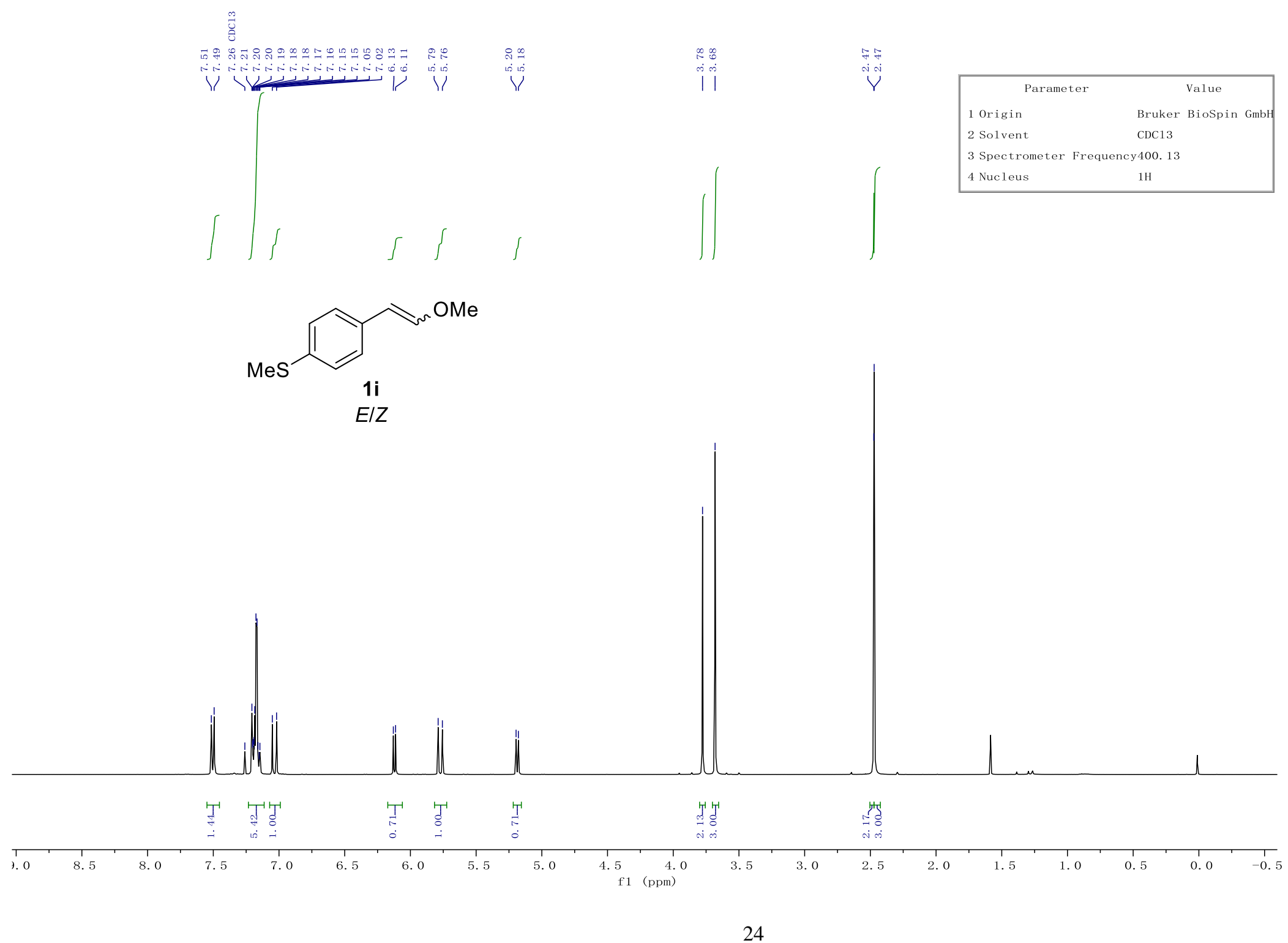



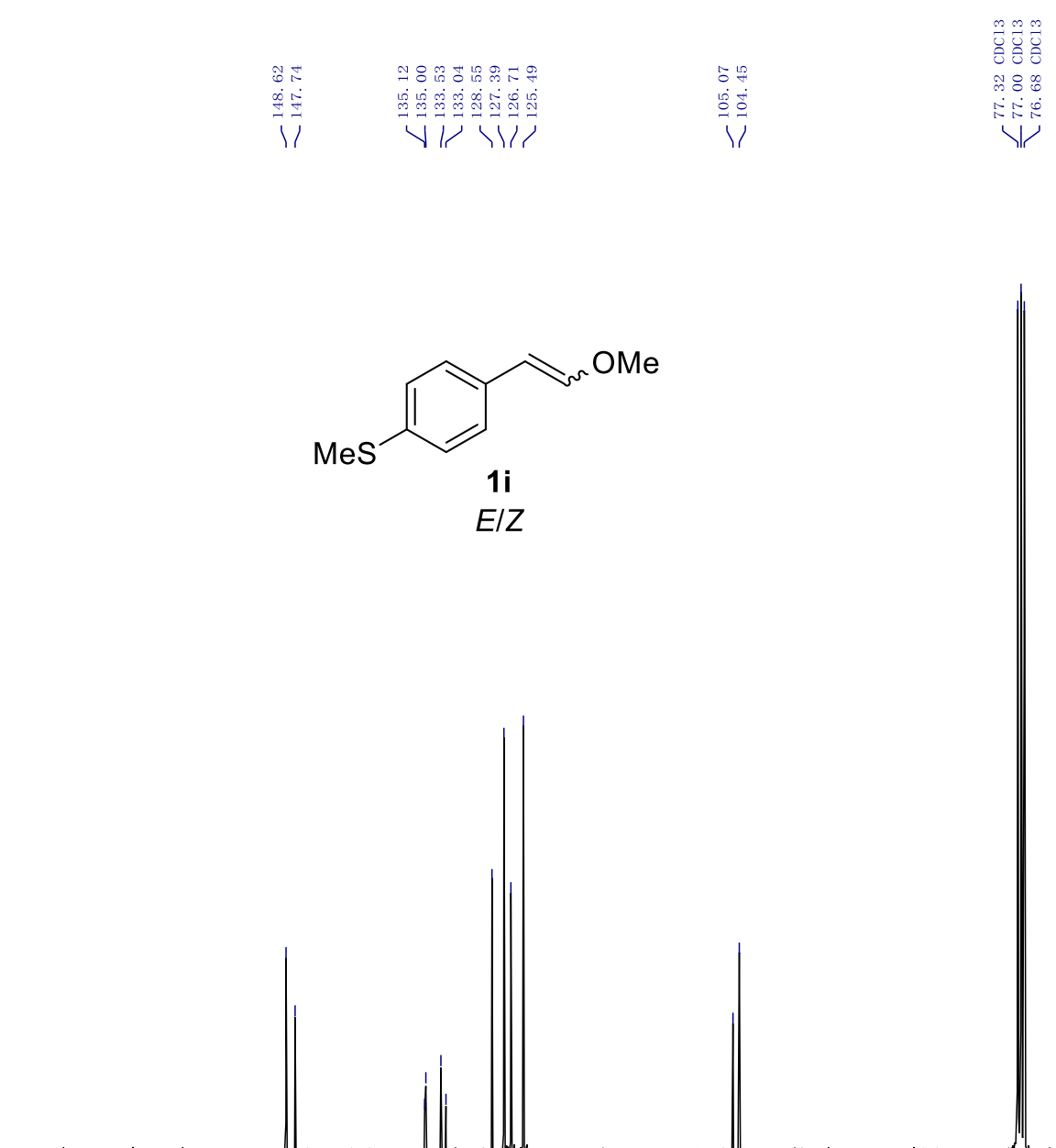

\section{00
$\dot{0} 0$
$\forall$
$V$}

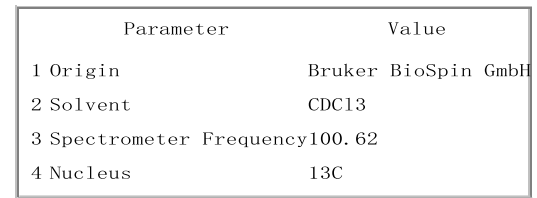

2 Solvent

4 Nucleus

$13 \mathrm{C}$

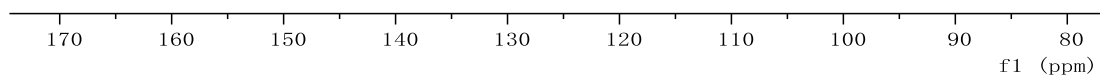




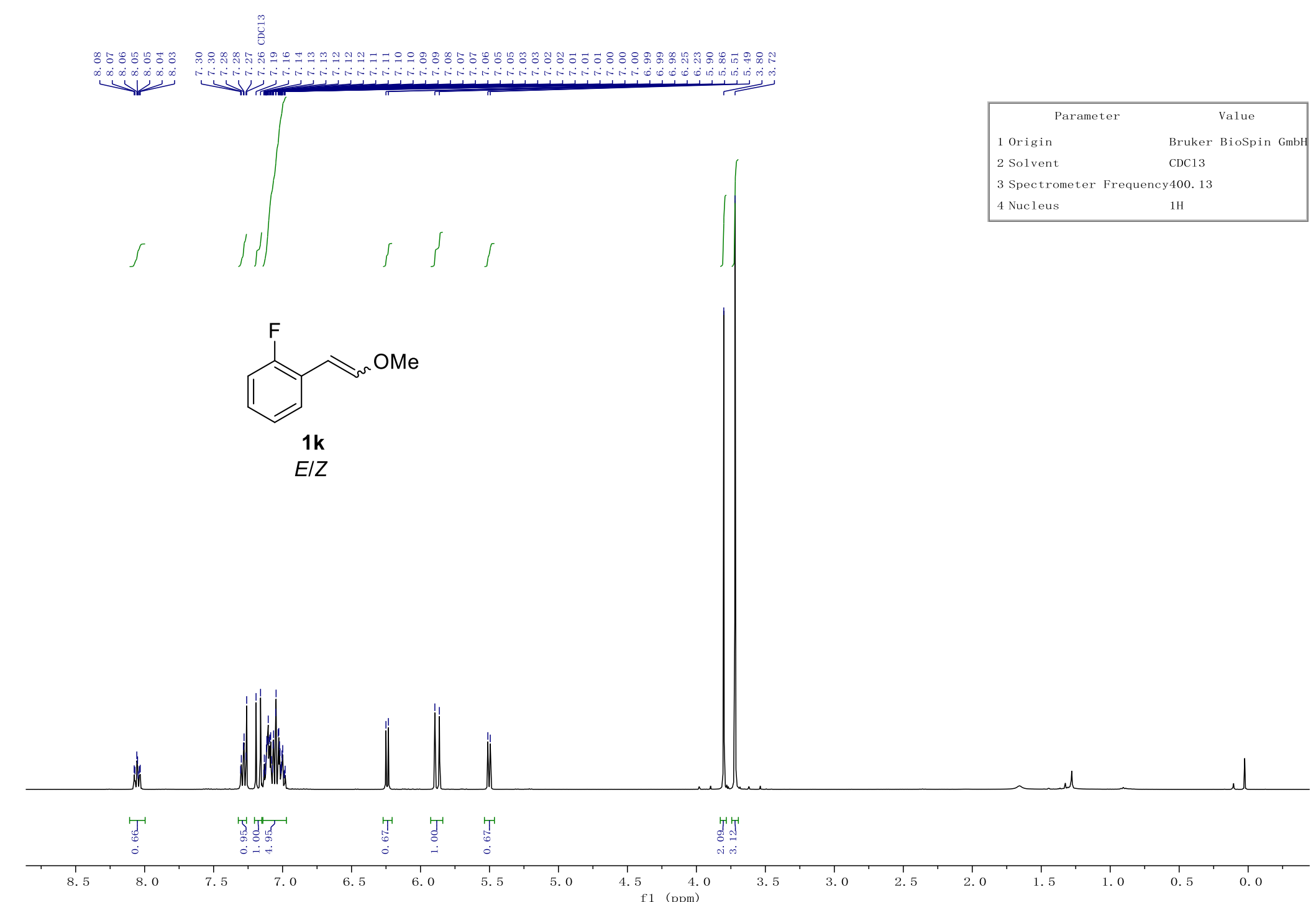




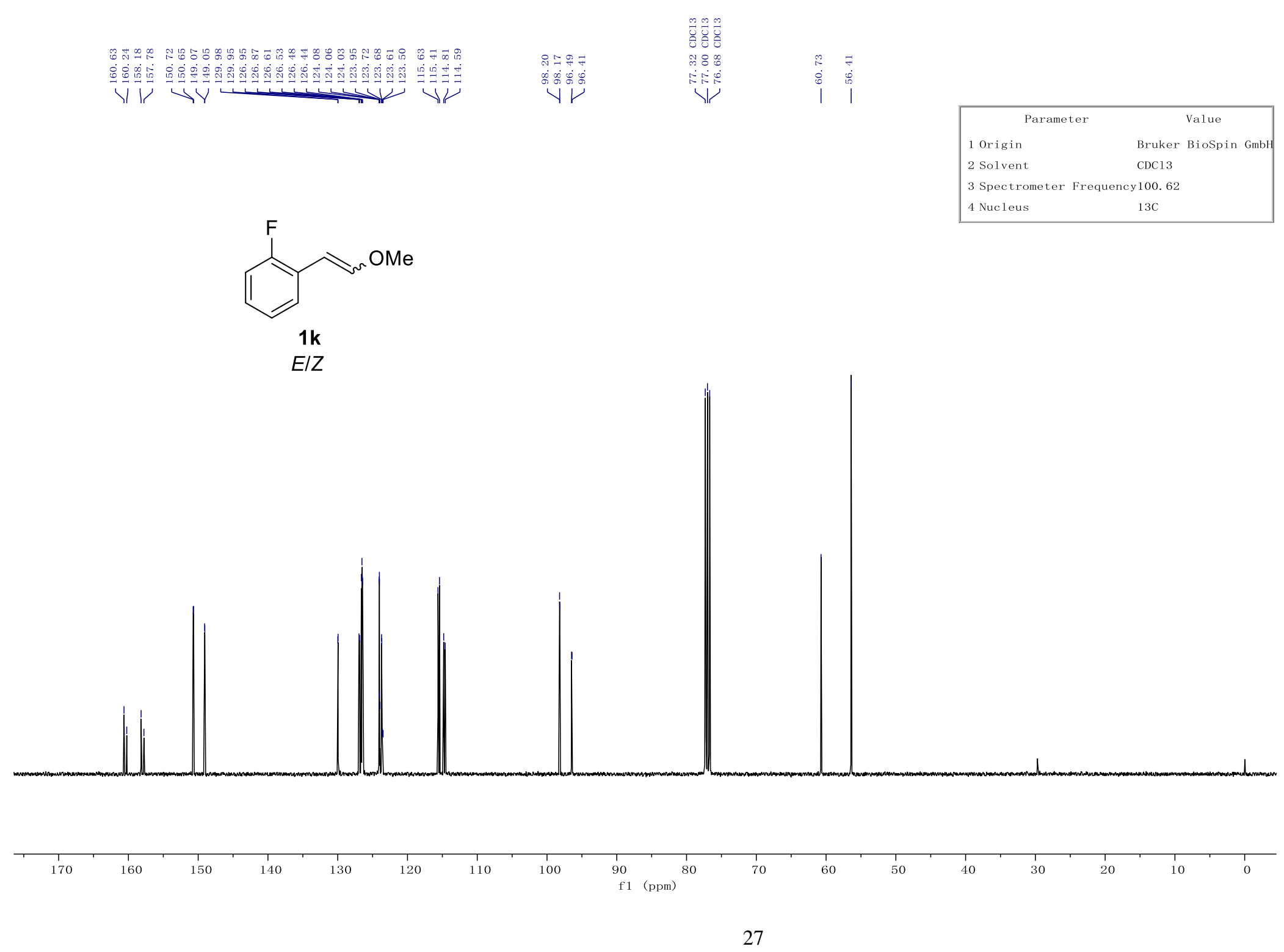






\begin{tabular}{|ll|}
\hline \multicolumn{1}{|c|}{ Parameter } & \multicolumn{1}{c|}{ Value } \\
1 Origin & Bruker BioSpin GmbH \\
2 Solvent & CDC13 \\
3 Spectrometer Frequency376. 46 \\
4 Nucleus & $19 \mathrm{~F}$ \\
\hline
\end{tabular}

$1 \mathbf{k}$
$E / Z$

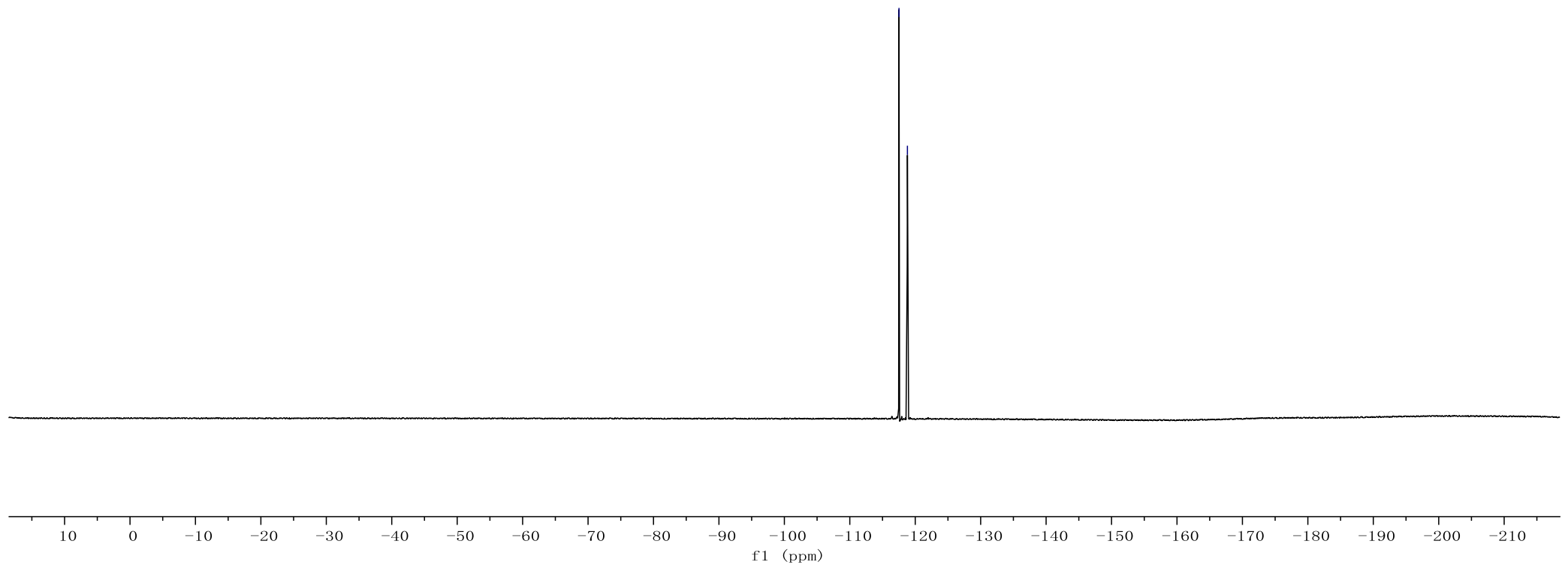




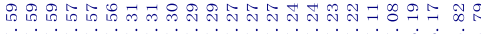

皮

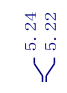<smiles>CCCCC</smiles><smiles>CCNC(=O)c1ccc(C=COC)cc1</smiles>

1n



$\mid$

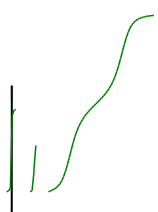

(n)

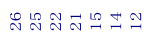

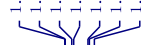

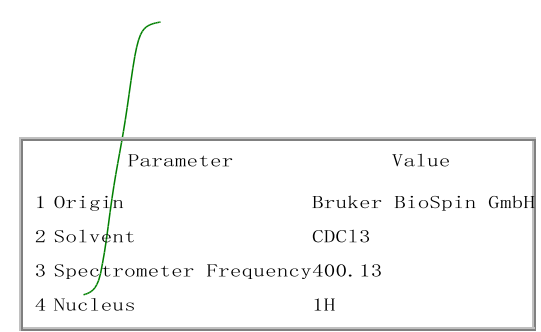







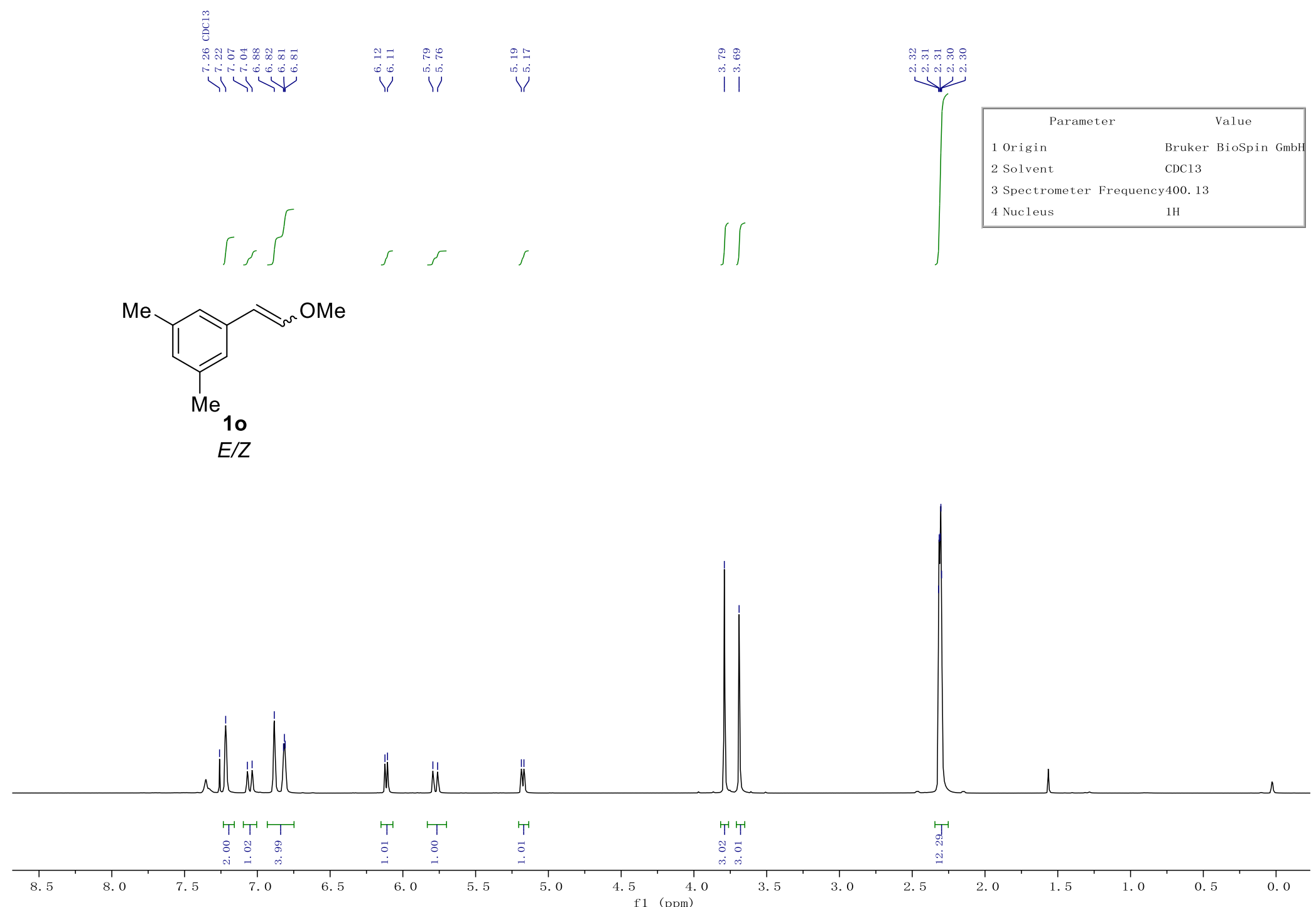

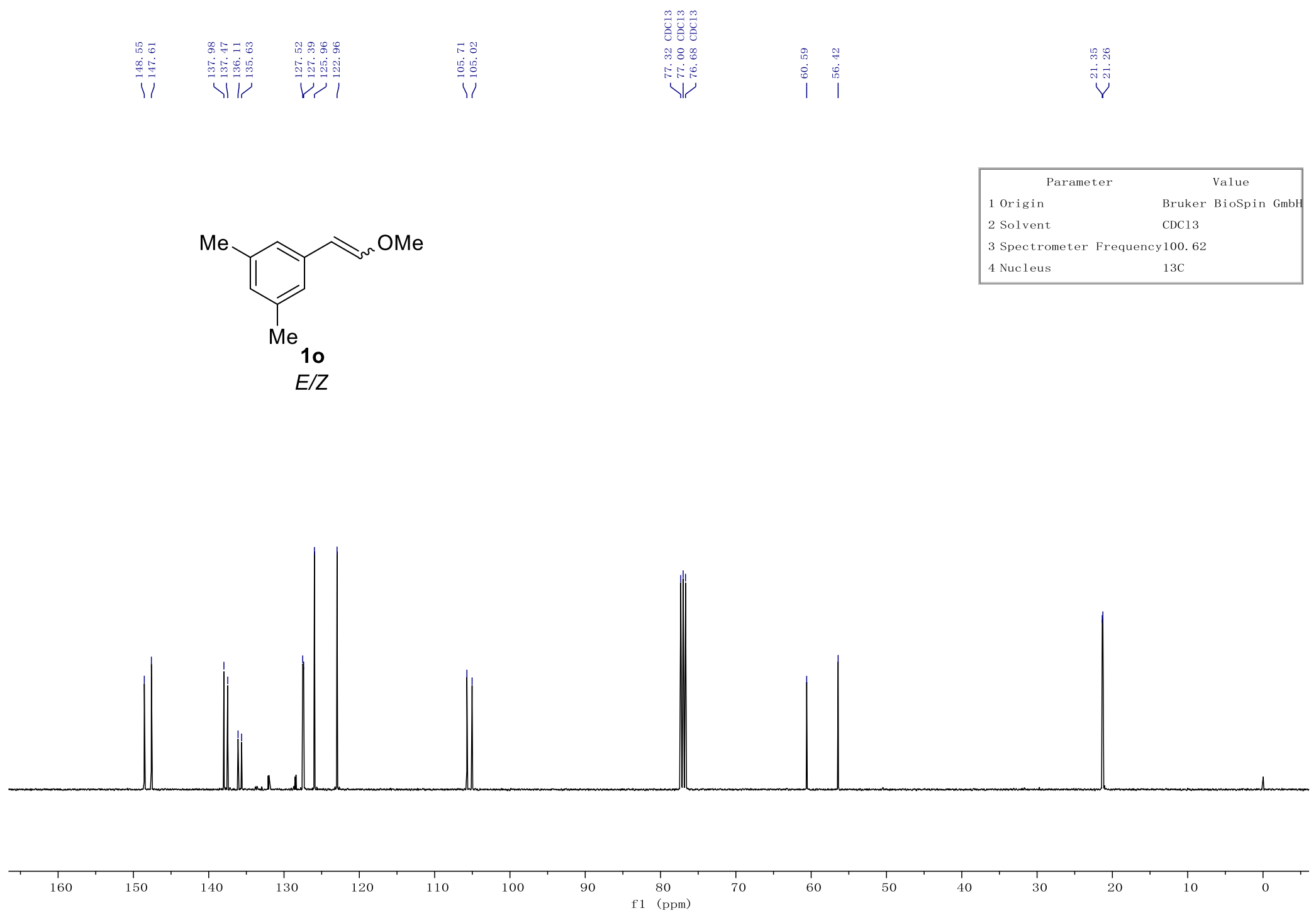


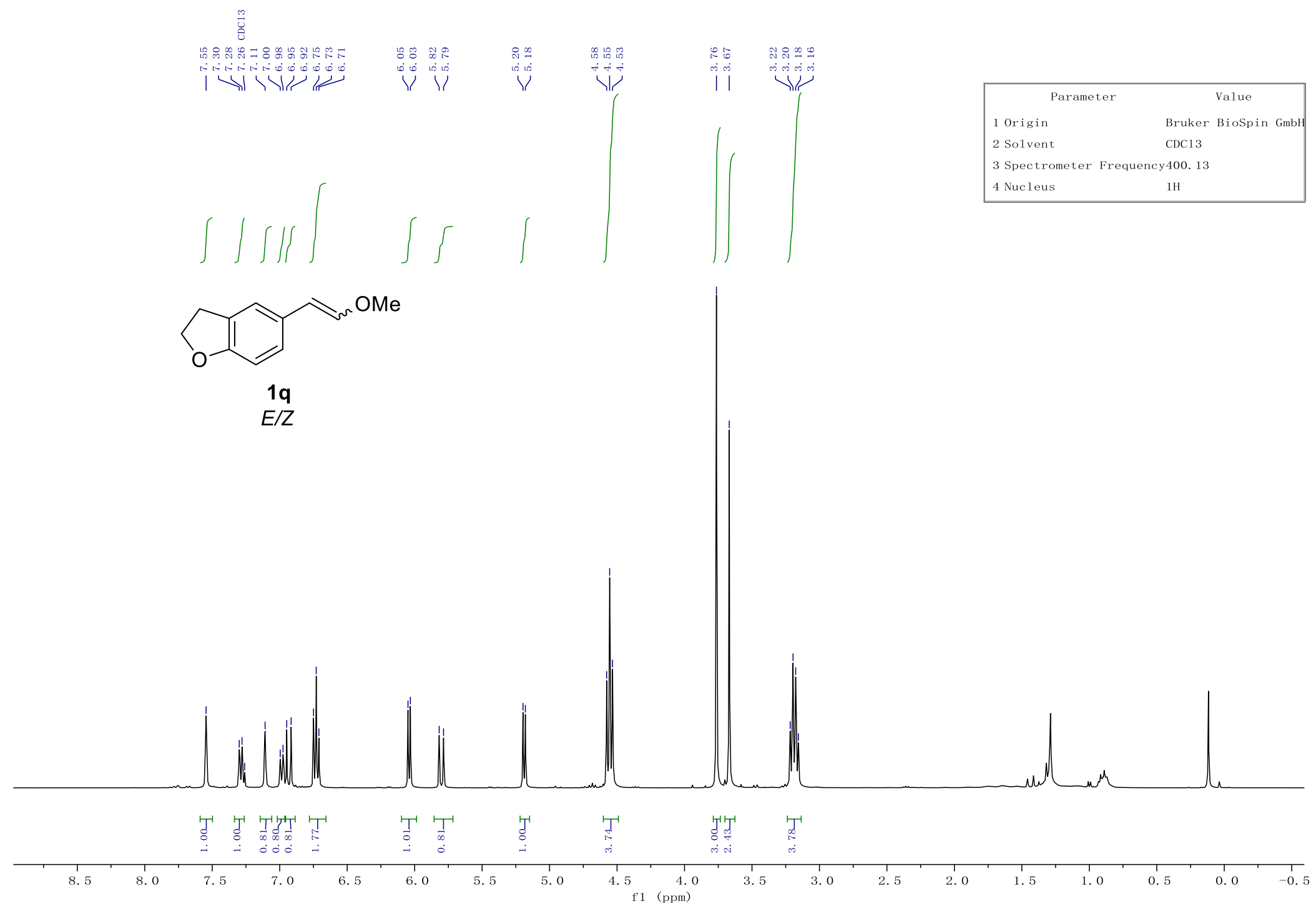









Vर्ण

\begin{tabular}{|c|c|}
\hline Parameter & Value \\
\hline 1 Origin & Bruker BioSpin GmbH \\
\hline 2 Solvent & $\mathrm{CDC} 13$ \\
\hline \multicolumn{2}{|c|}{3 Spectrometer Frequency 100.62} \\
\hline
\end{tabular}
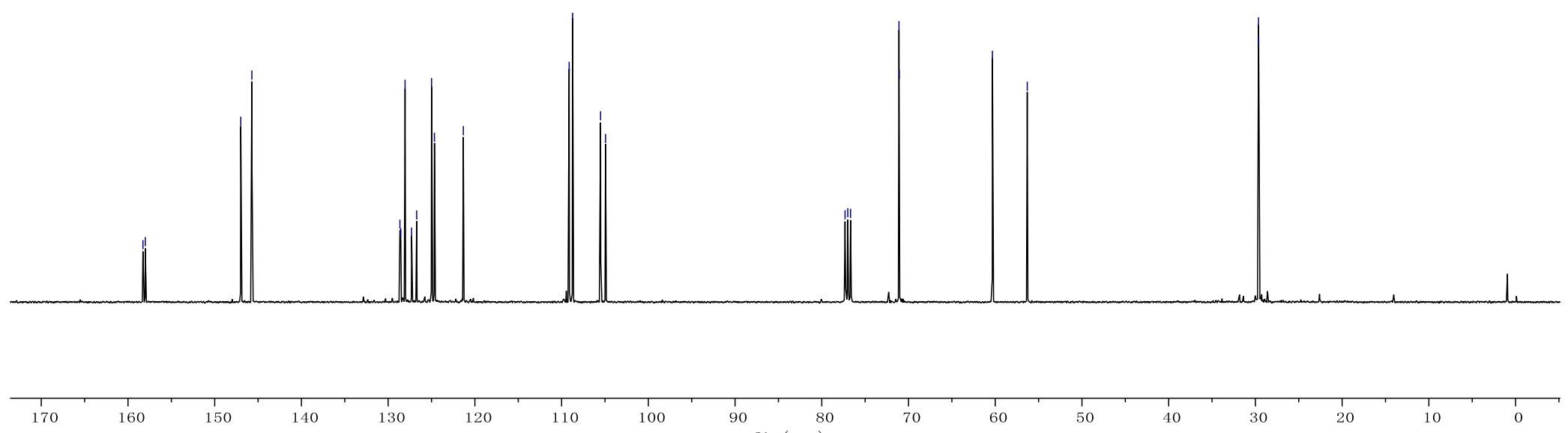

150

140

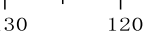

100 $90 \quad 80$ 


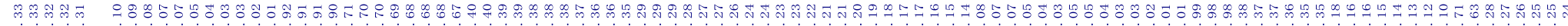

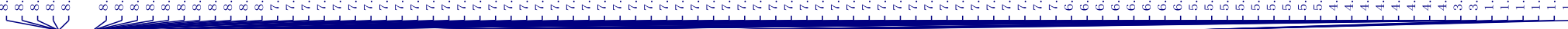

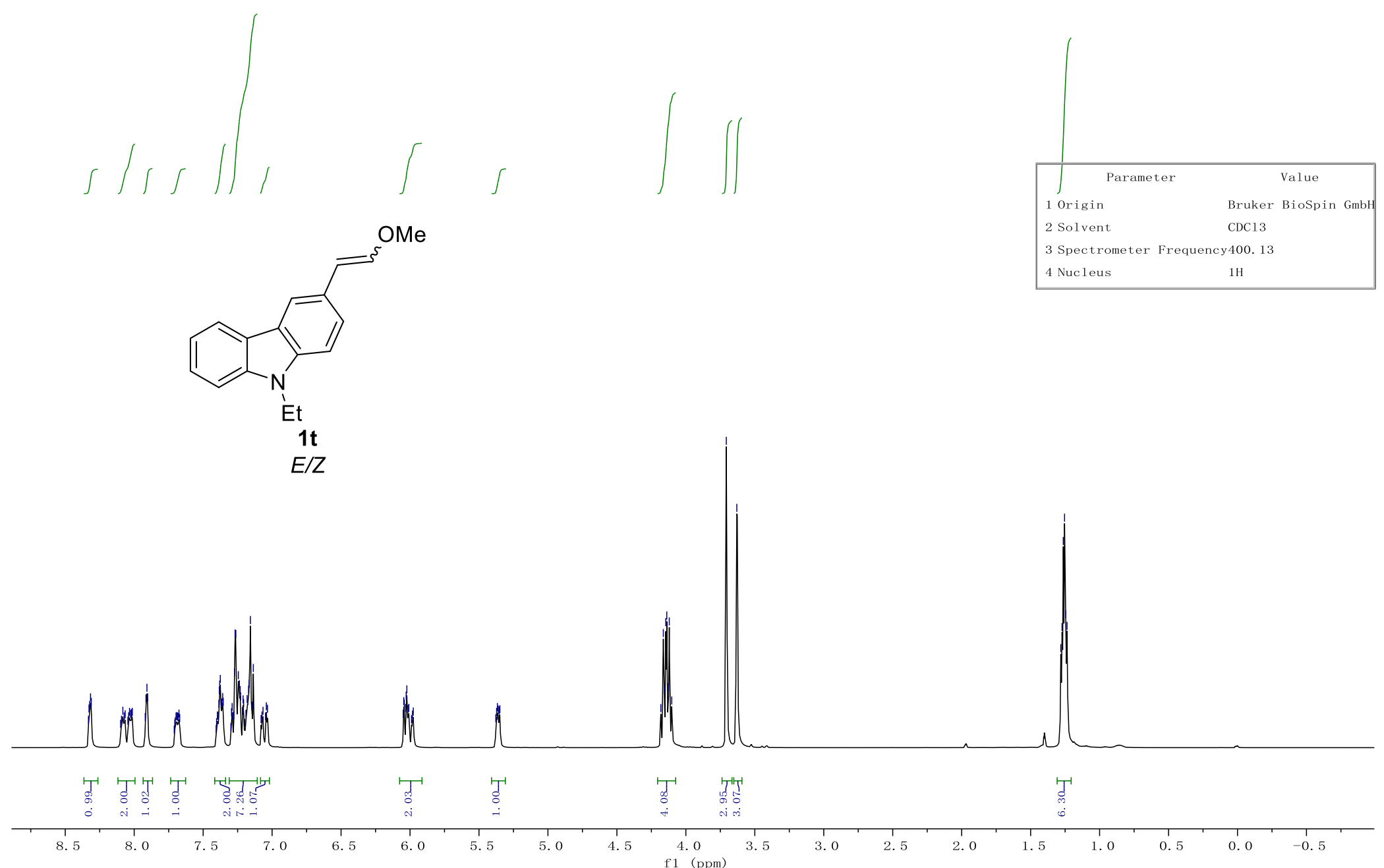






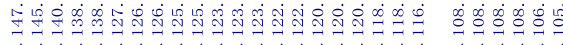

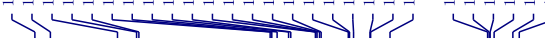

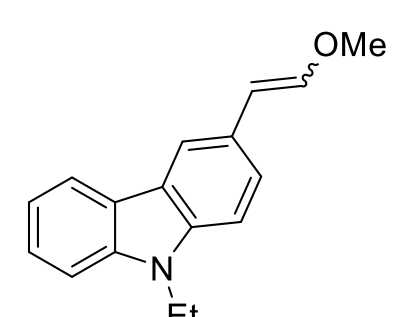

$1 \mathrm{t}$
$E / Z$
器器

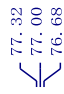

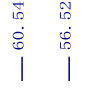

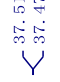

\begin{tabular}{|lc|}
\hline \multicolumn{1}{|c|}{ Parameter } & \multicolumn{2}{c|}{ Value } \\
1 Origin & Bruker BioSpin GmbH \\
2 Solvent & CDC13 \\
3 Spectrometer Frequency 100. 62 \\
4 Nucleus & $13 \mathrm{C}$ \\
\hline
\end{tabular}

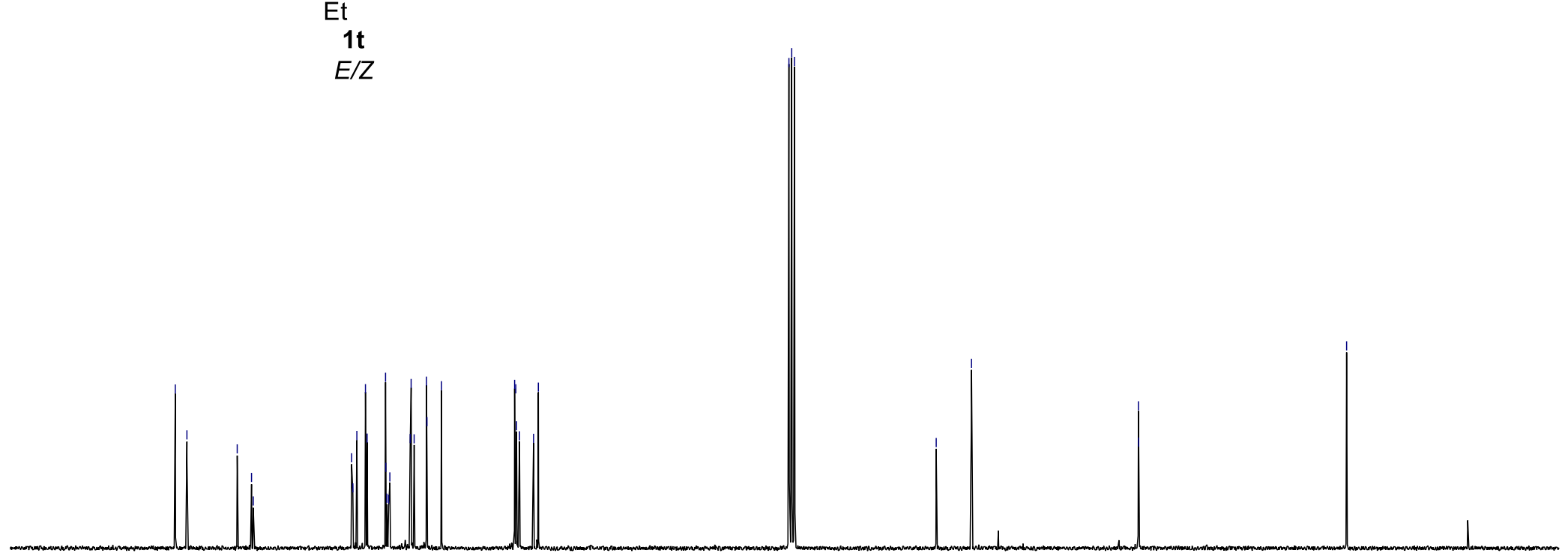




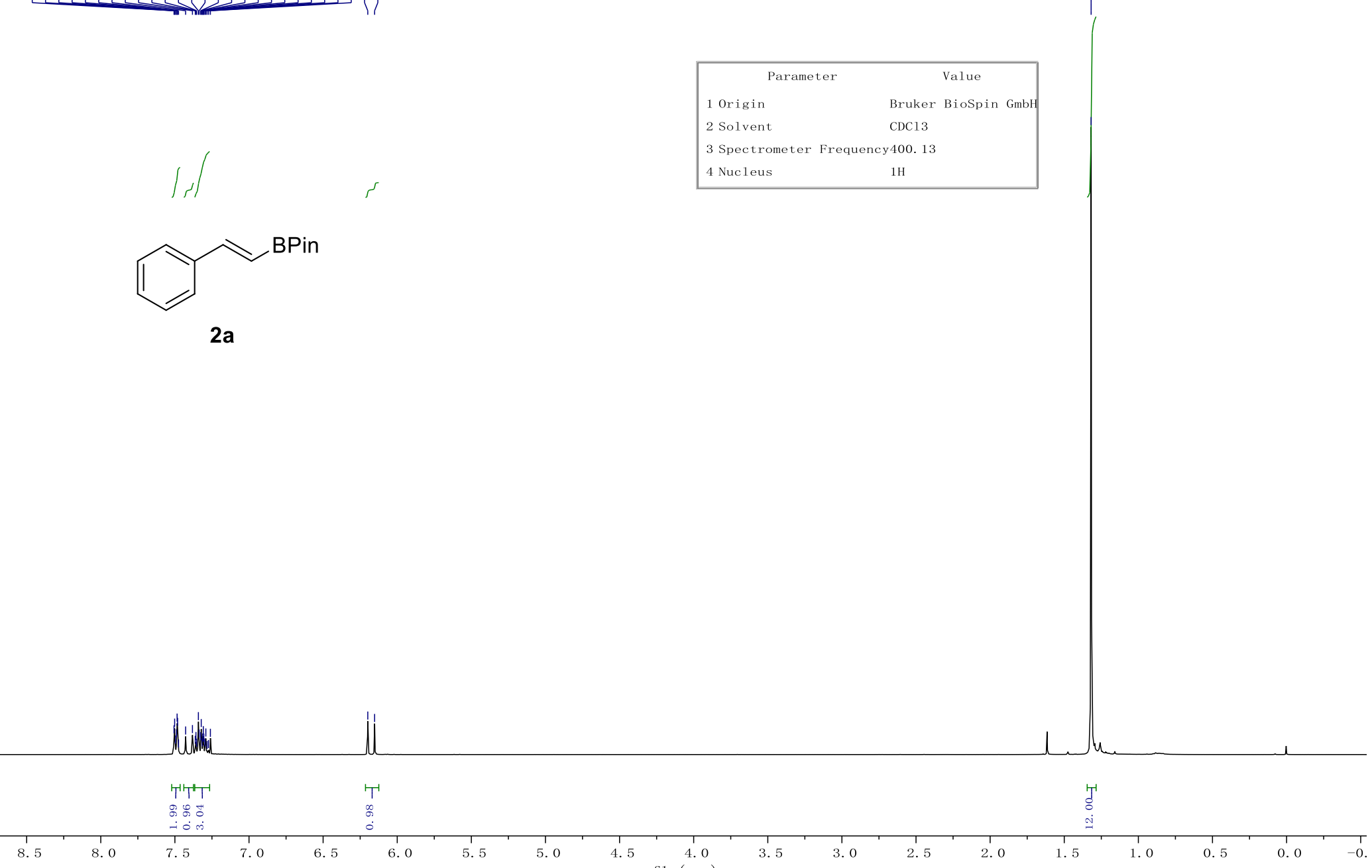



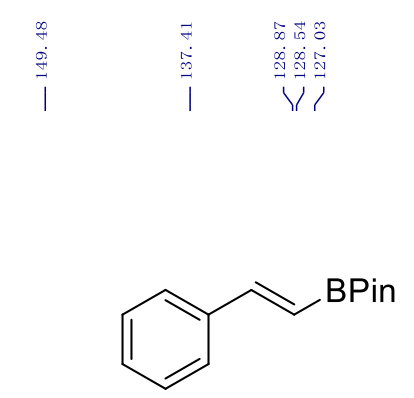

$2 a$
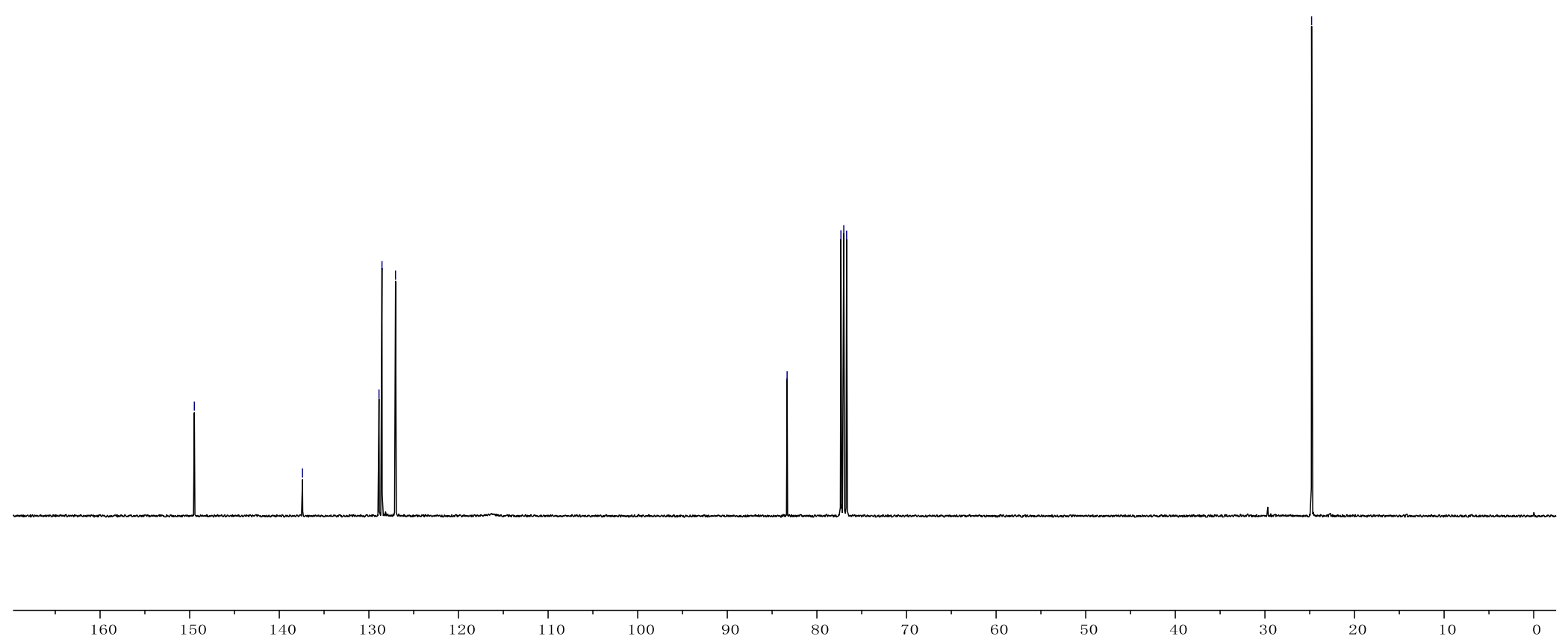

160

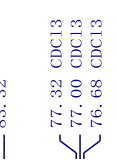

\begin{tabular}{|cc|}
\hline \multicolumn{1}{|c|}{ Parameter } & Value \\
1 Origin & Bruker BioSpin GmbH
\end{tabular}

2 Solvent

3 Spectrometer Frequency 100.62
4 Nucleus
$13 \mathrm{C}$ $13 \mathrm{C}$
CDC13 


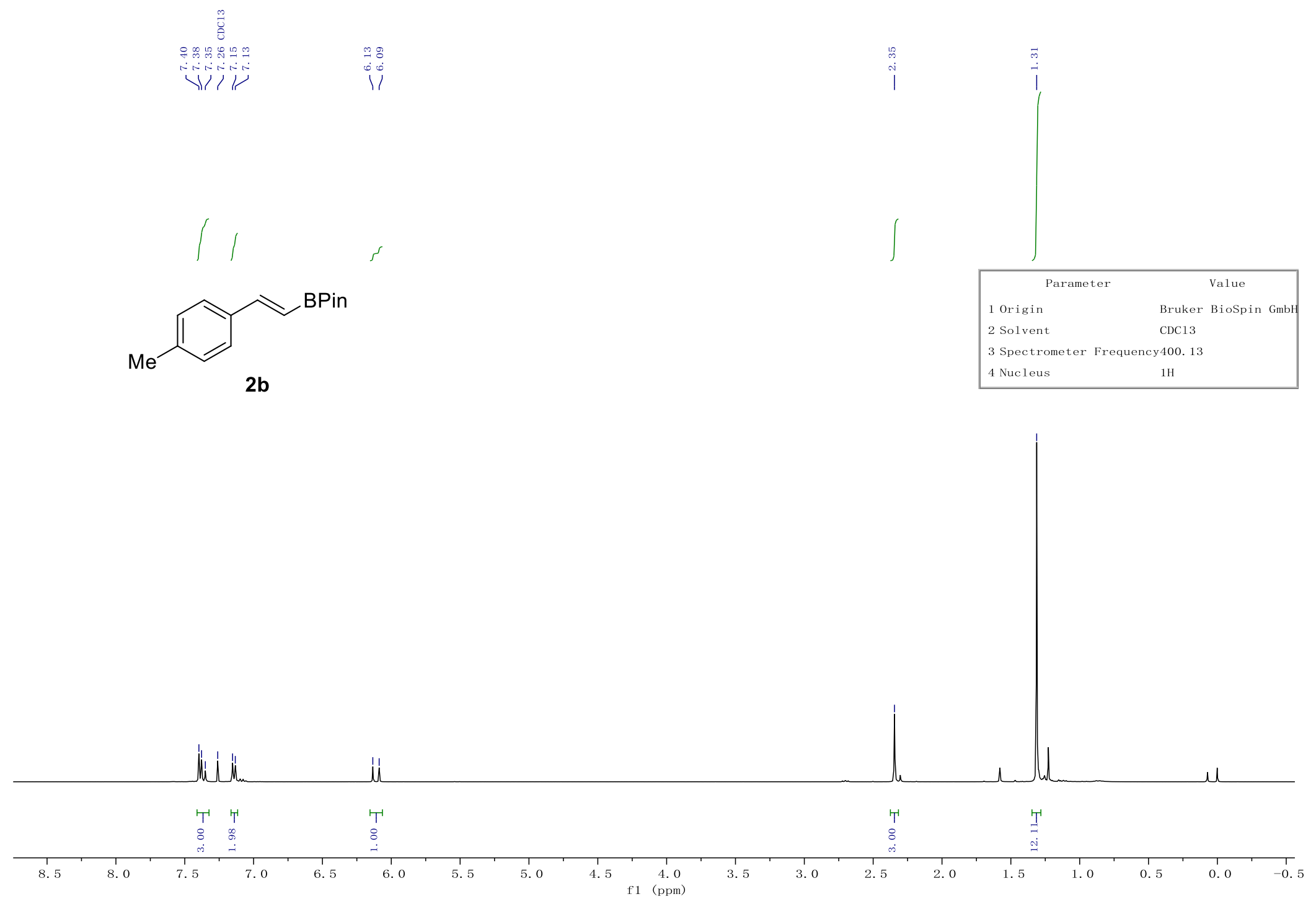




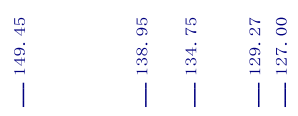

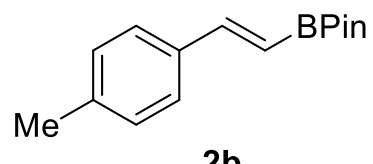

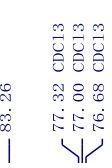

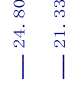

\begin{tabular}{|ll|}
\hline \multicolumn{1}{|c|}{ Parameter } & \multicolumn{2}{c|}{ Value } \\
1 Origin & Bruker BioSpin GmbH \\
2 Solvent & CDC13 \\
3 Spectrometer Frequency 100. 62 \\
4 Nucleus & $13 \mathrm{C}$ \\
\hline
\end{tabular}

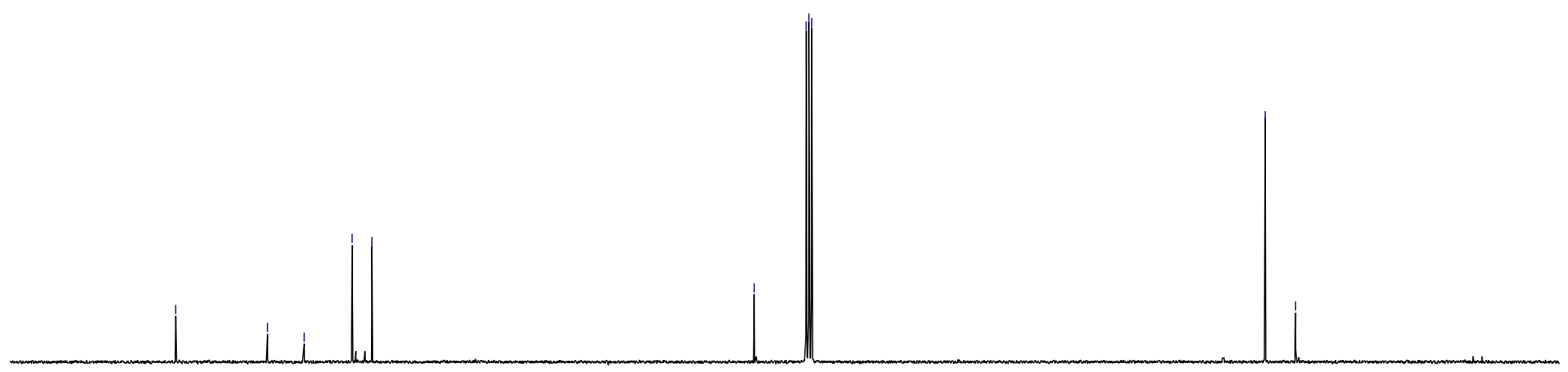




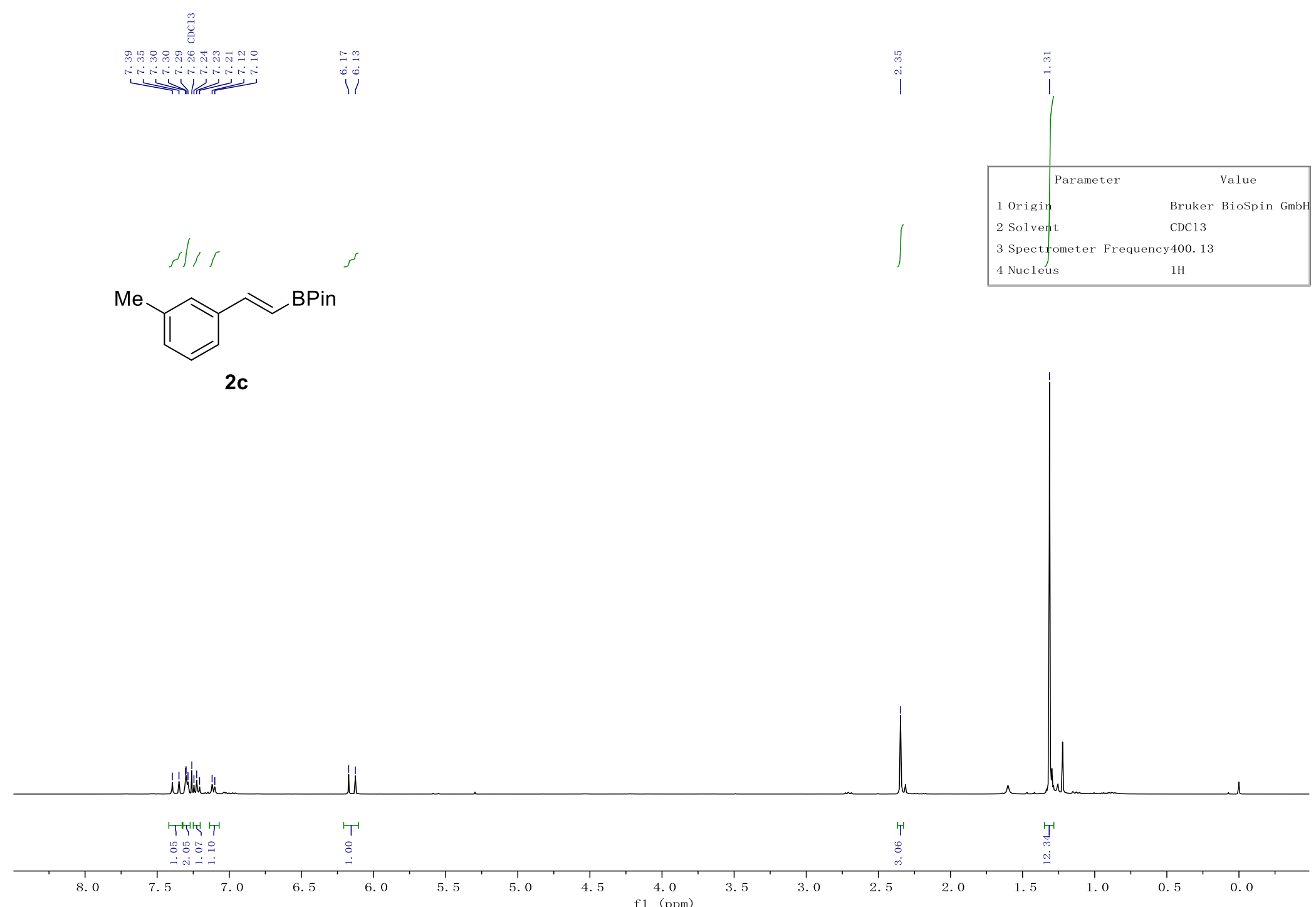






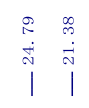

\begin{tabular}{|lc|}
\hline \multicolumn{1}{|c|}{ Parameter } & \multicolumn{1}{c|}{ Value } \\
1 Origin & Bruker BioSpin GmbH \\
2 Solvent & CDC13 \\
3 Spectrometer Frequency 100. 62 \\
4 Nucleus & $13 \mathrm{C}$ \\
\hline
\end{tabular}



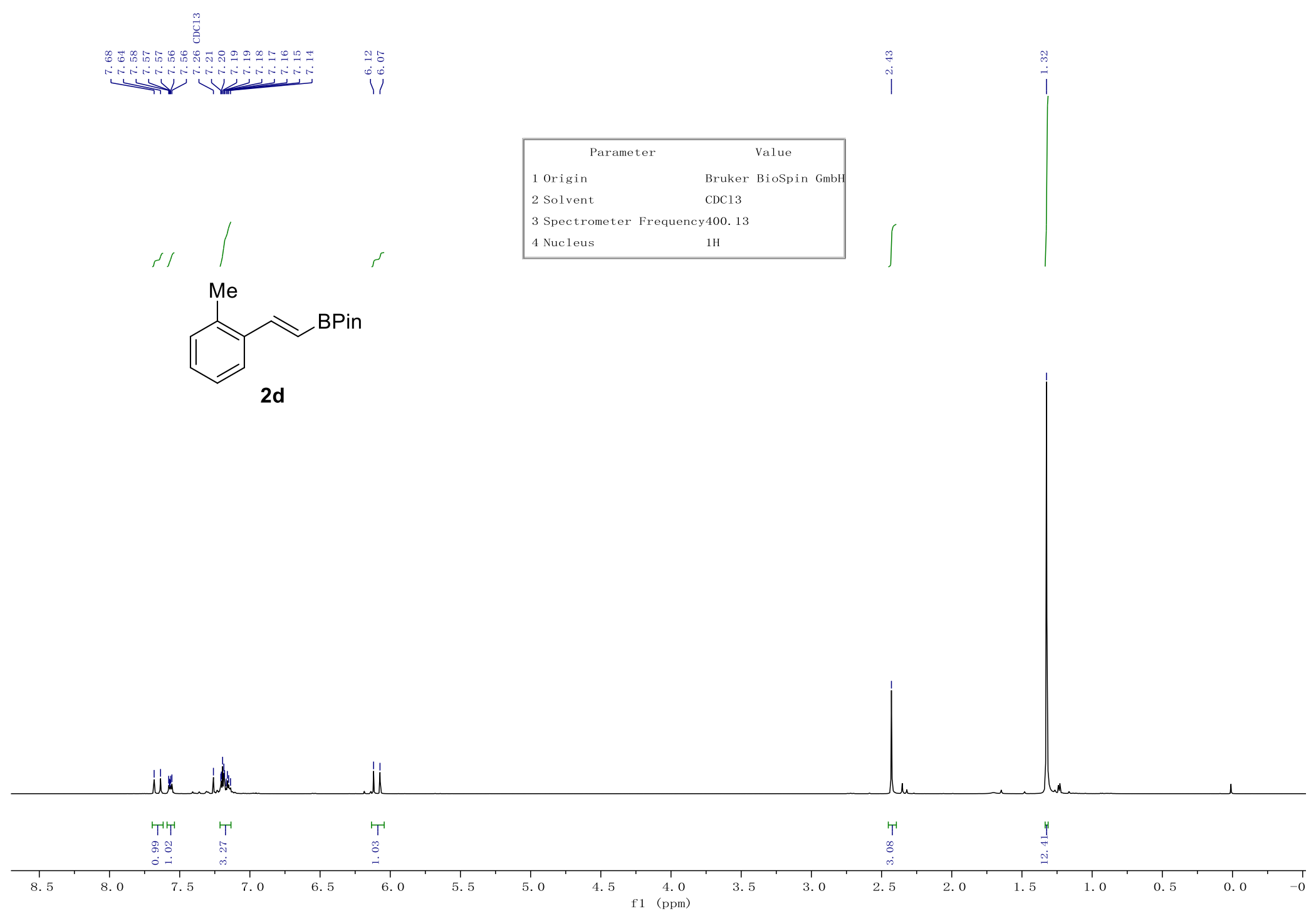




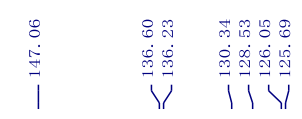

$\underbrace{M e}_{2 \mathbf{d}} B P i n$

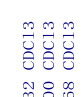

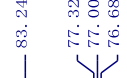

\begin{tabular}{|lc|}
\hline \multicolumn{1}{|c|}{ Parameter } & \multicolumn{2}{c|}{ Value } \\
1 Origin & Bruker BioSpin GmbH \\
2 Solvent & CDC13 \\
3 Spectrometer & Frequency 100. 62 \\
4 4 Nucleus & $13 \mathrm{C}$ \\
\hline
\end{tabular}
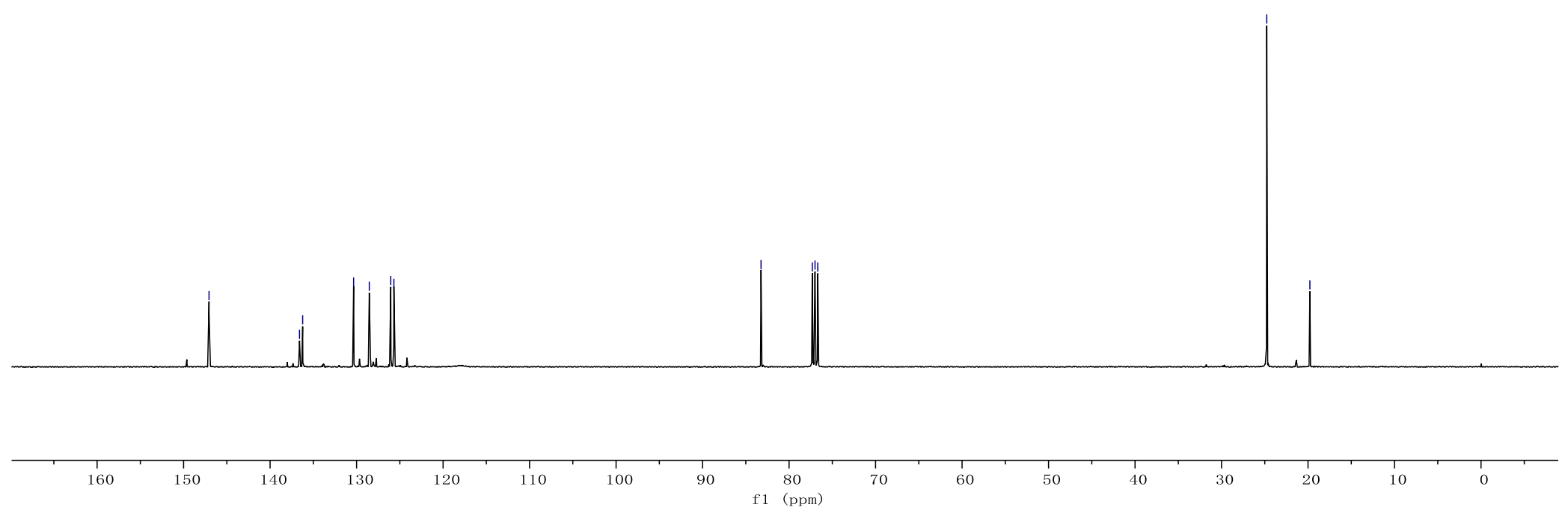


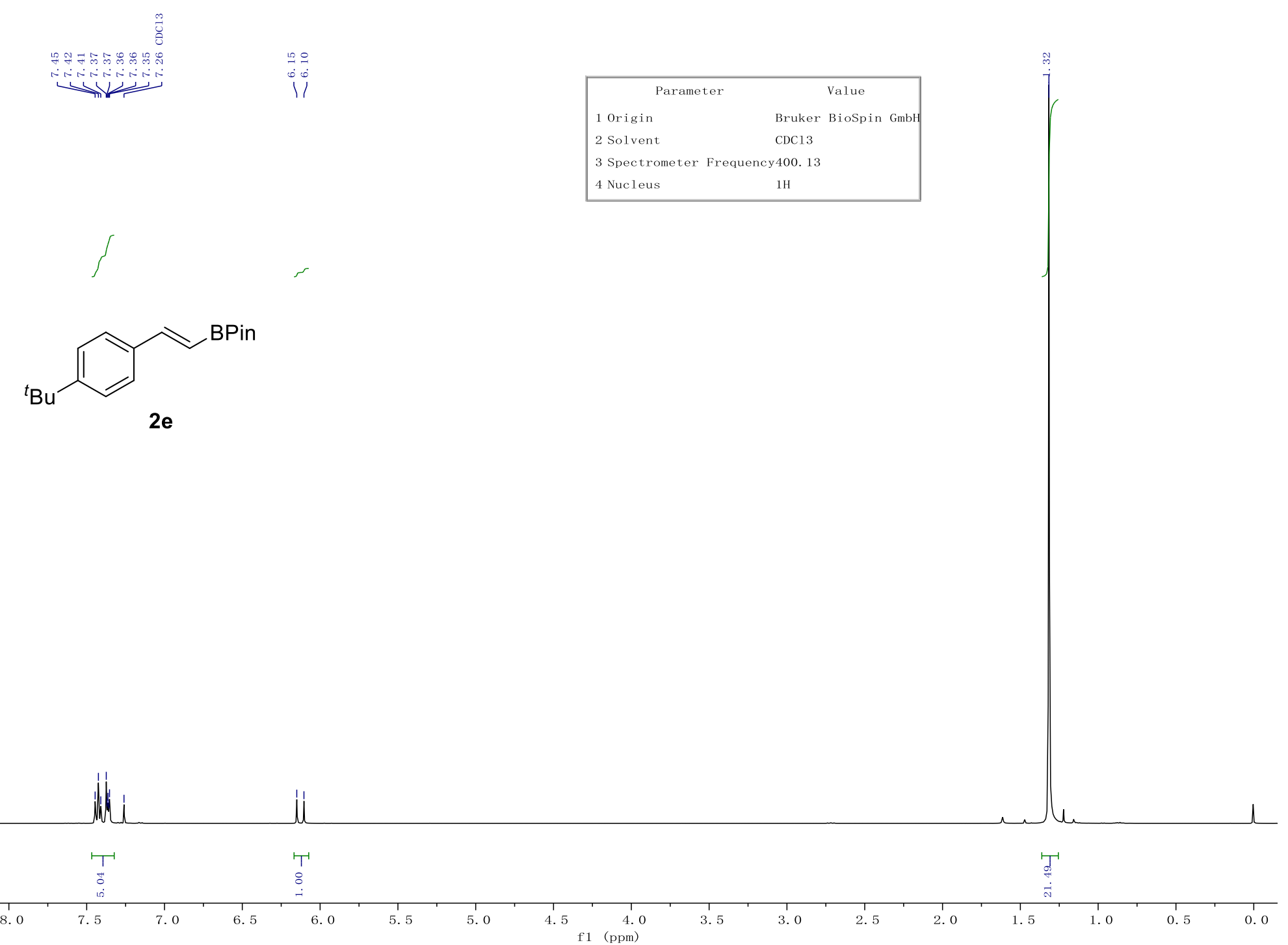




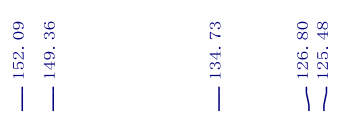

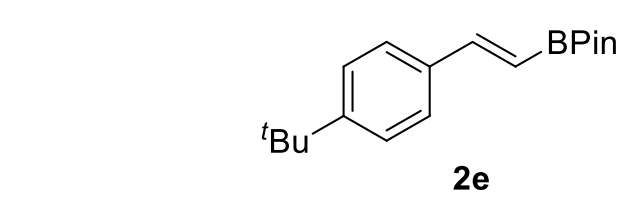

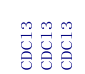

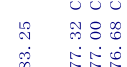

|

\begin{tabular}{|lc|}
\hline \multicolumn{1}{|c|}{ Parameter } & Value \\
1 Origin & Bruker BioSpin GmbH \\
2 Solvent & CDC13 \\
3 Spectrometer & Frequency 100. 62 \\
4 Nucleus & 13C \\
\hline
\end{tabular}

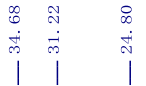
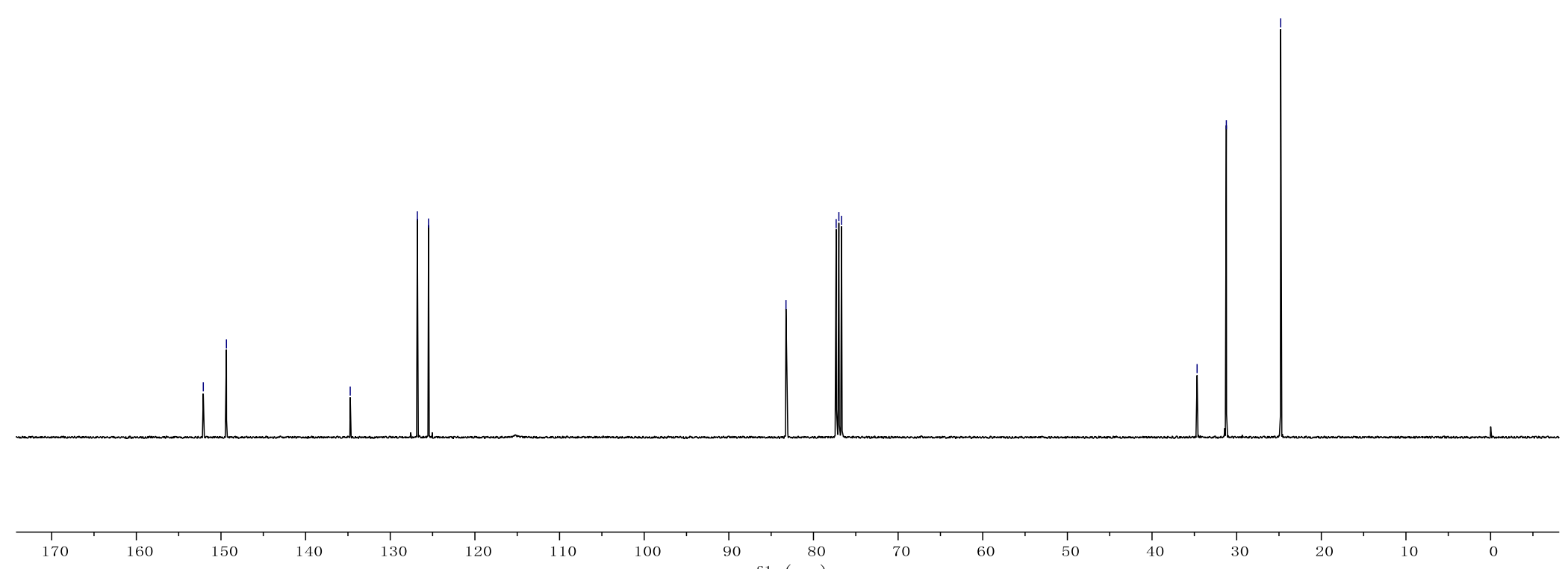

140

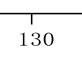

120

100 $90 \quad 80$ 






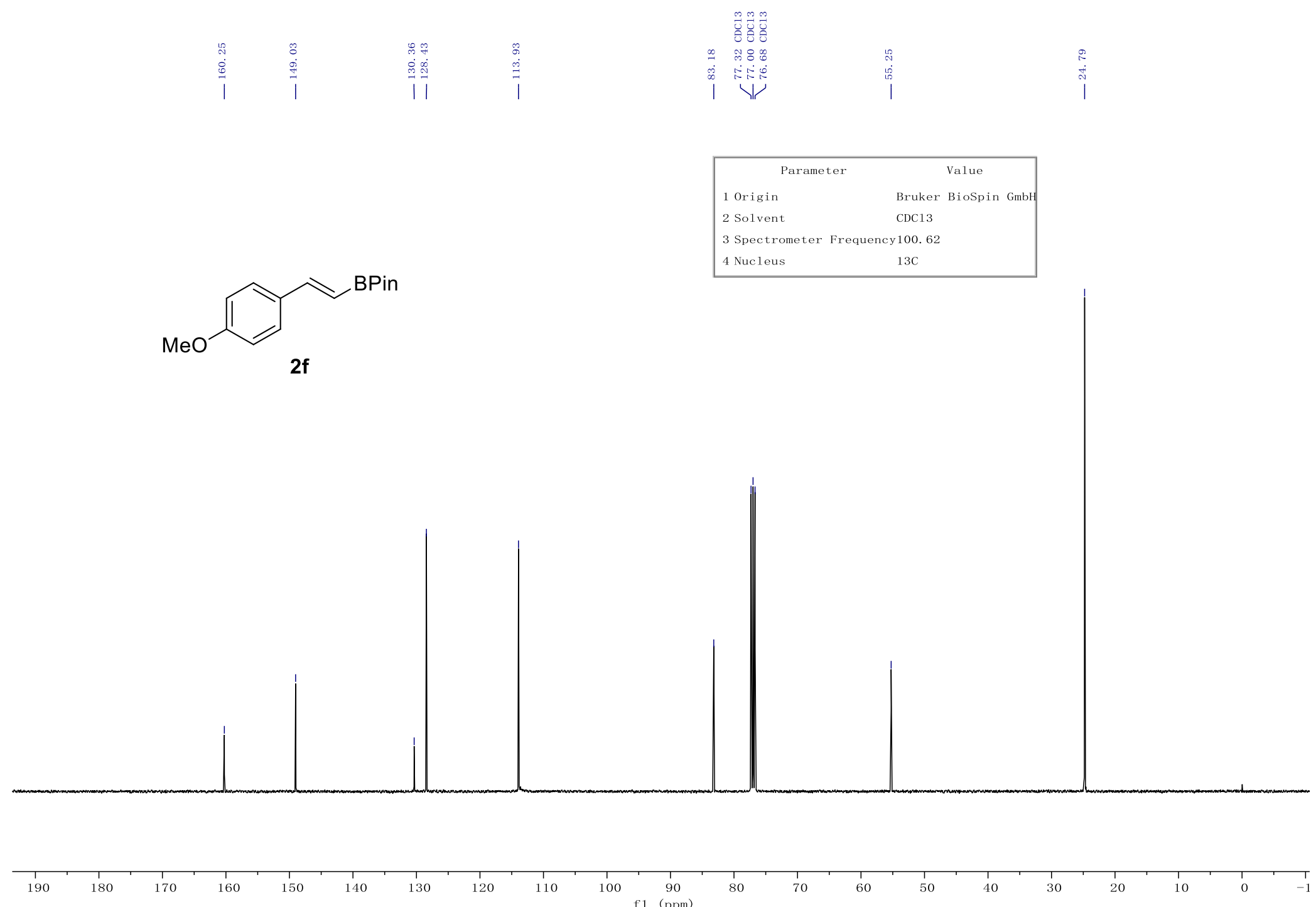


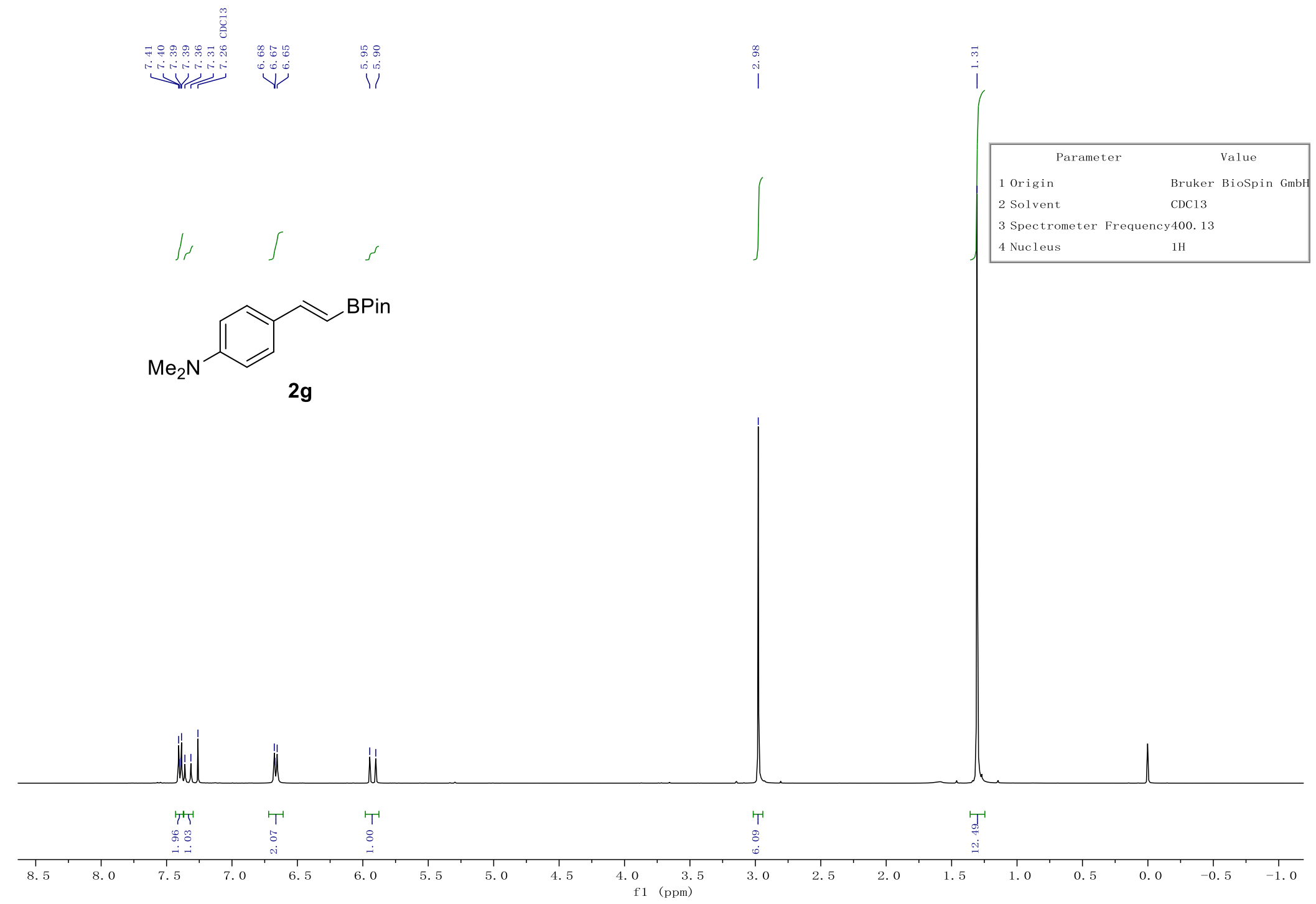




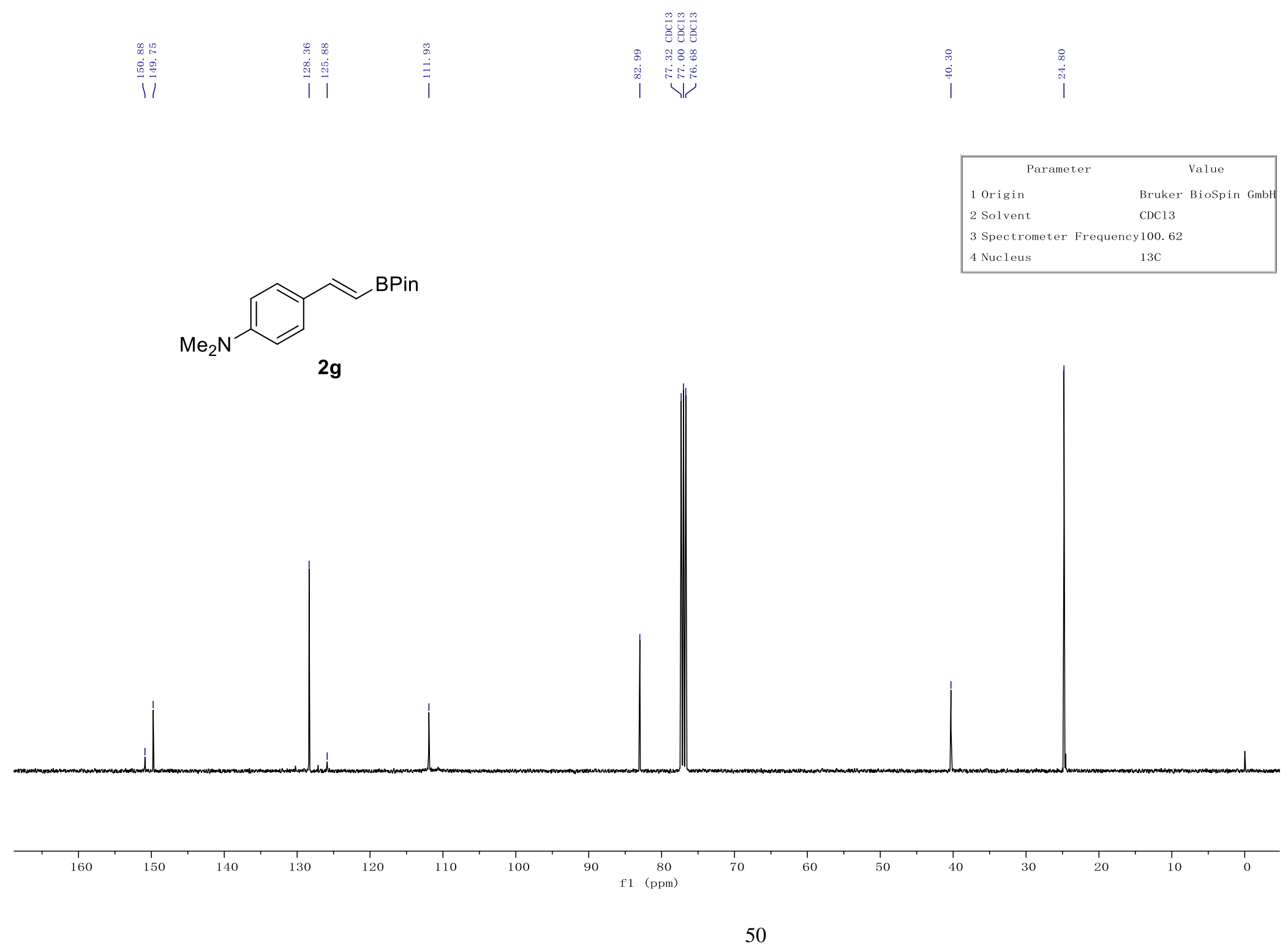


思

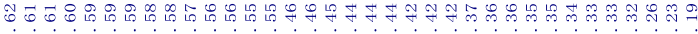

istrictiviog

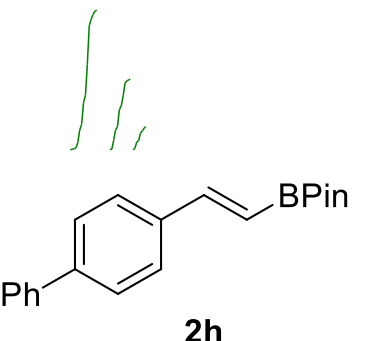

$2 \mathrm{~h}$

\begin{tabular}{|ll|}
\hline \multicolumn{1}{|c|}{ Parameter } & \multicolumn{1}{c|}{ Value } \\
1 Origin & Bruker BioSpin GmbH \\
2 Solvent & CDC13 \\
3 Spectrometer Frequency 400.13 \\
4 Nucleus & $1 \mathrm{H}$ \\
\hline
\end{tabular}

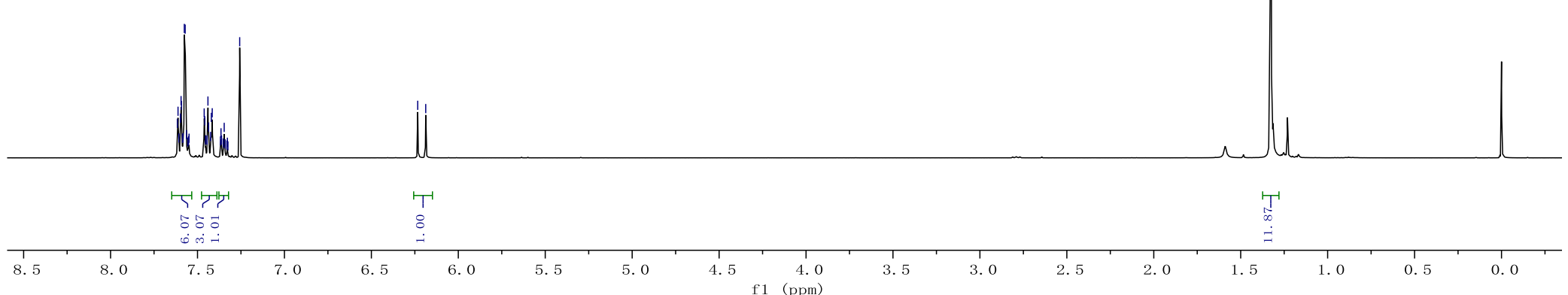




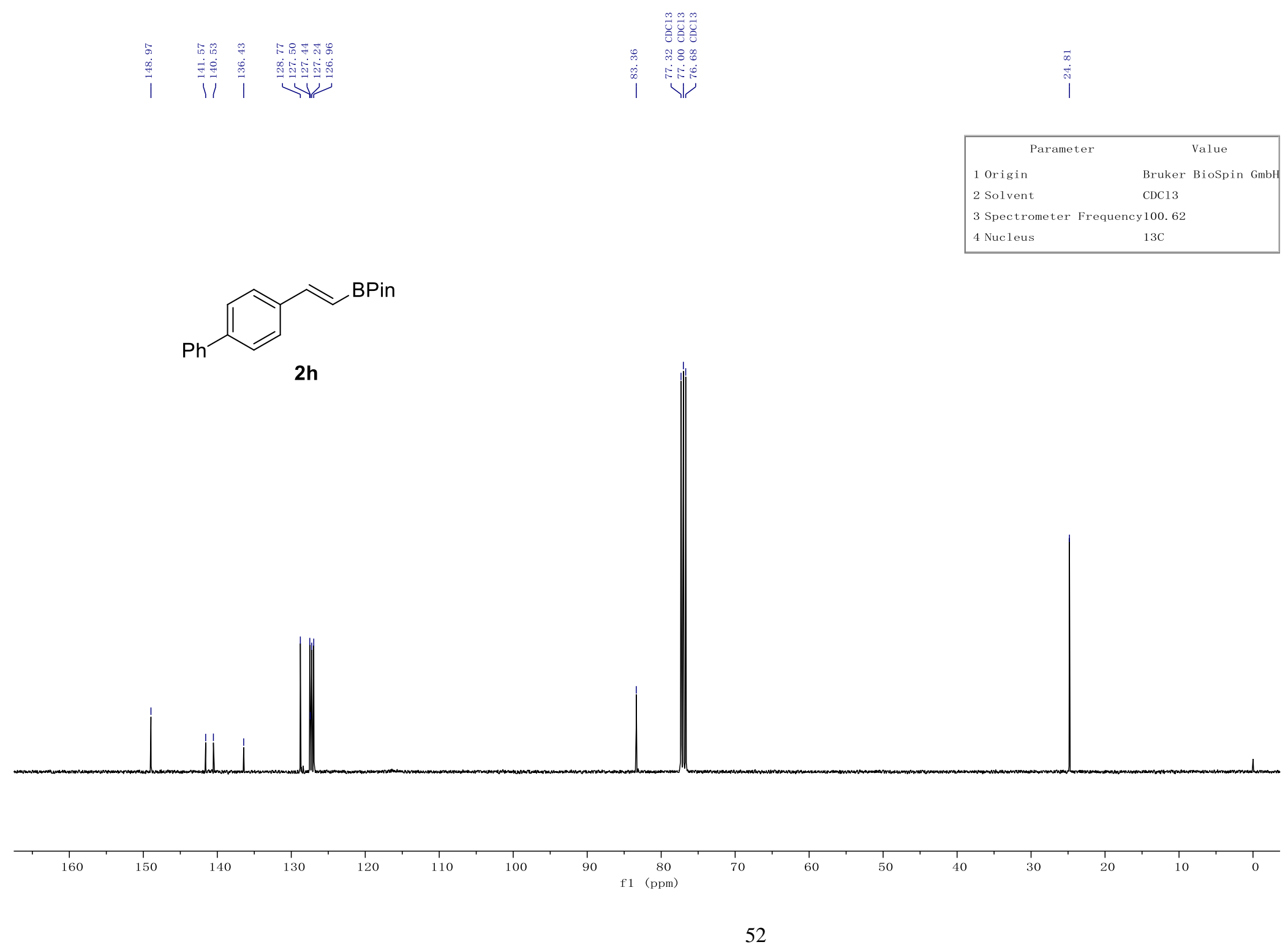




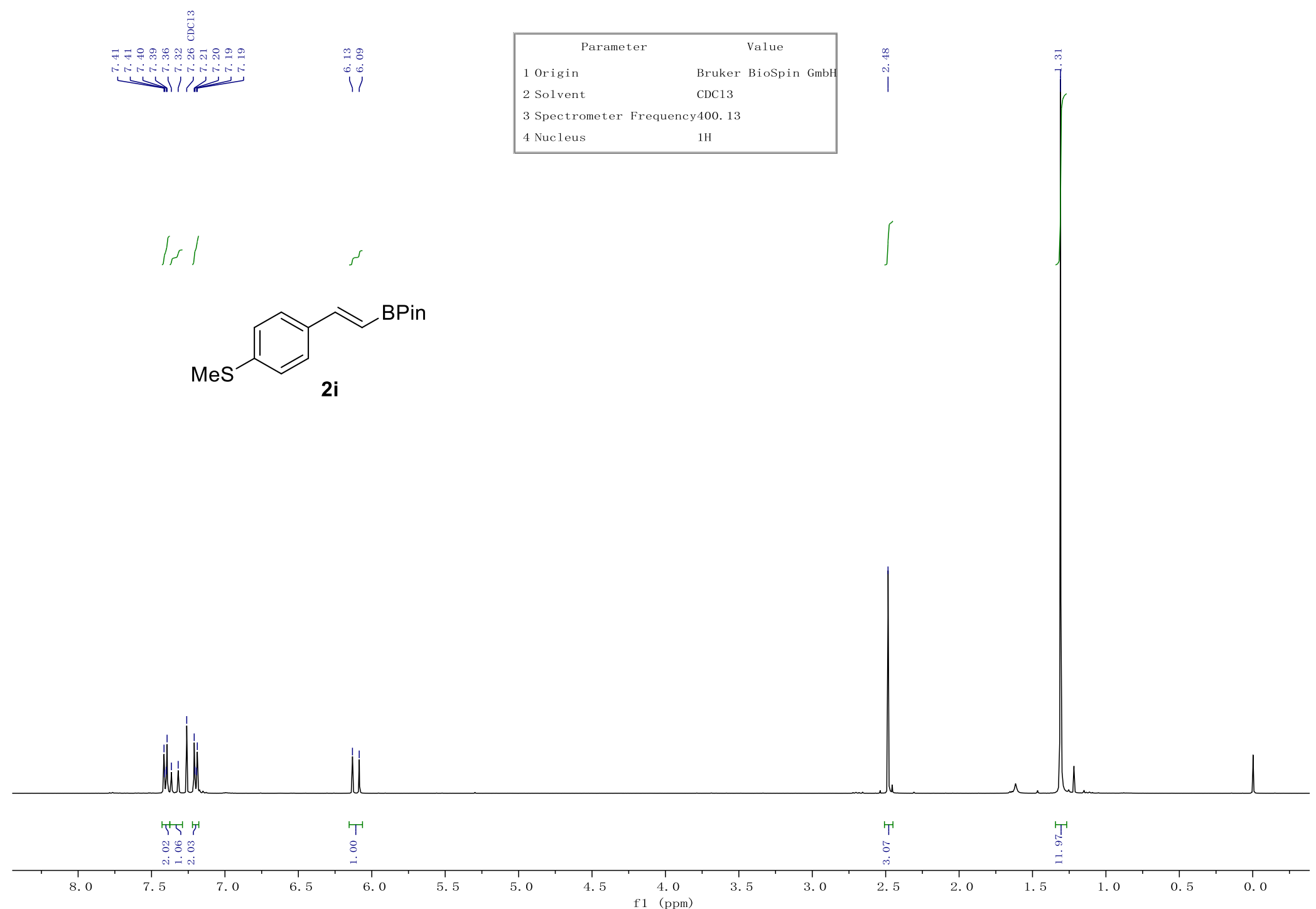









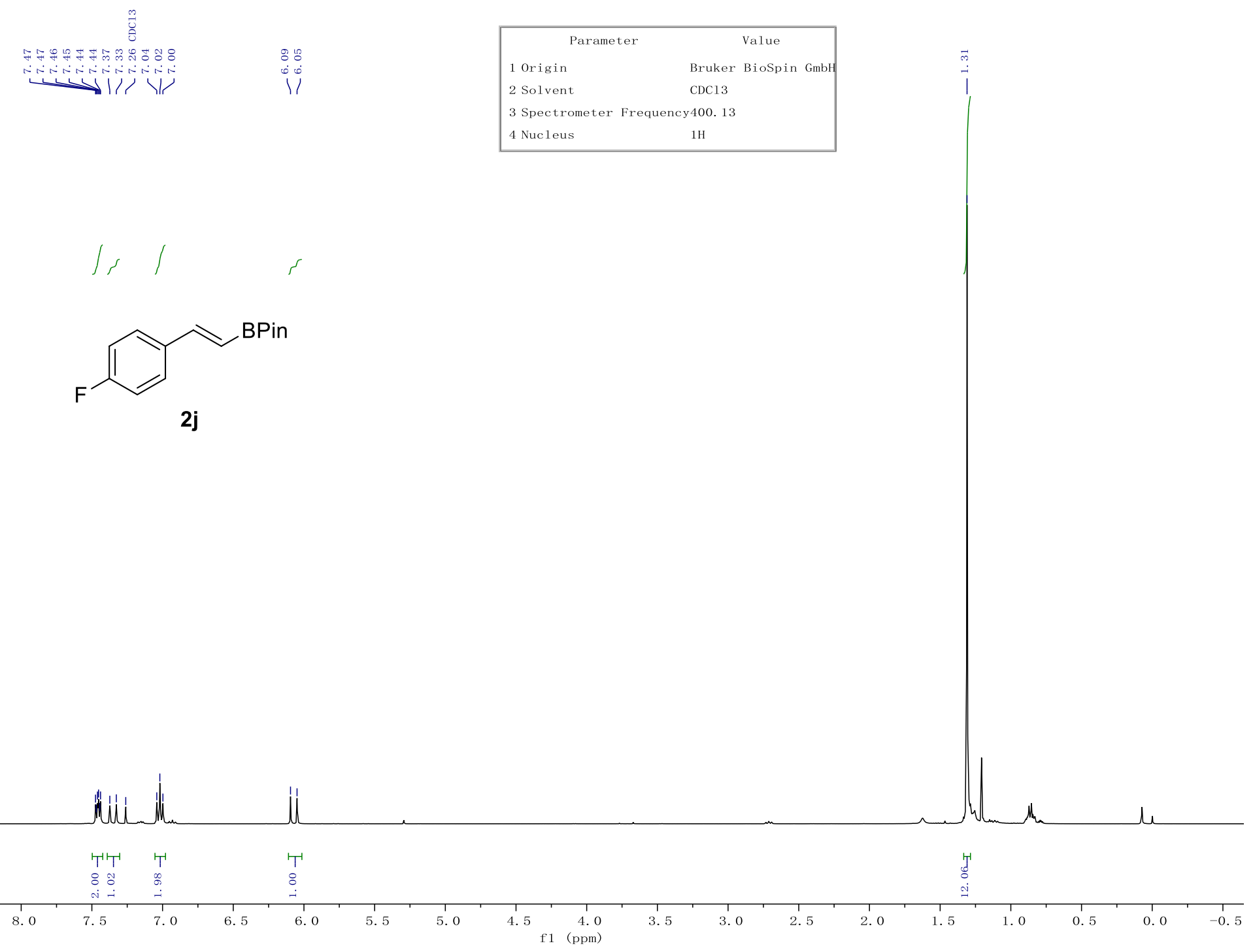



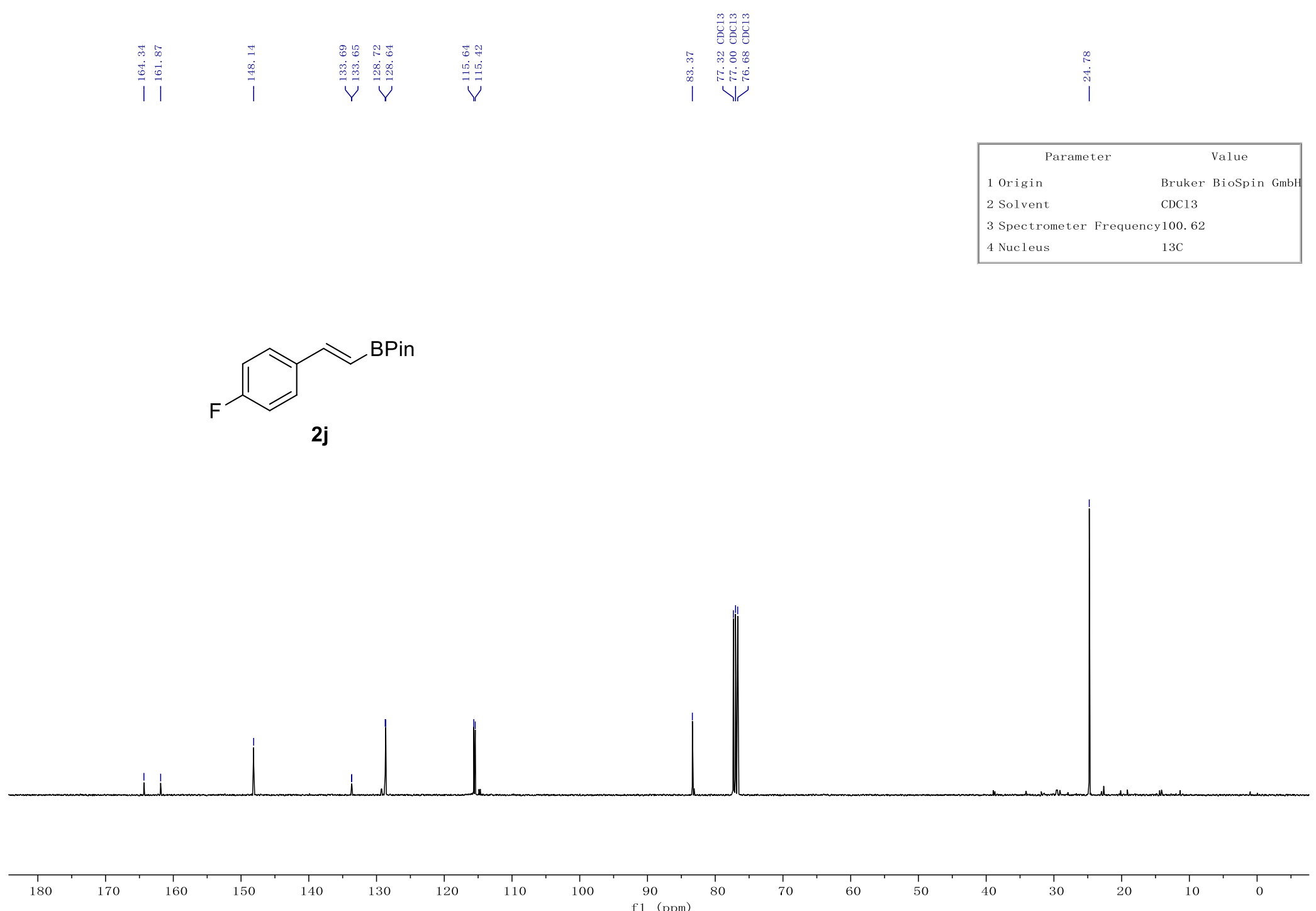




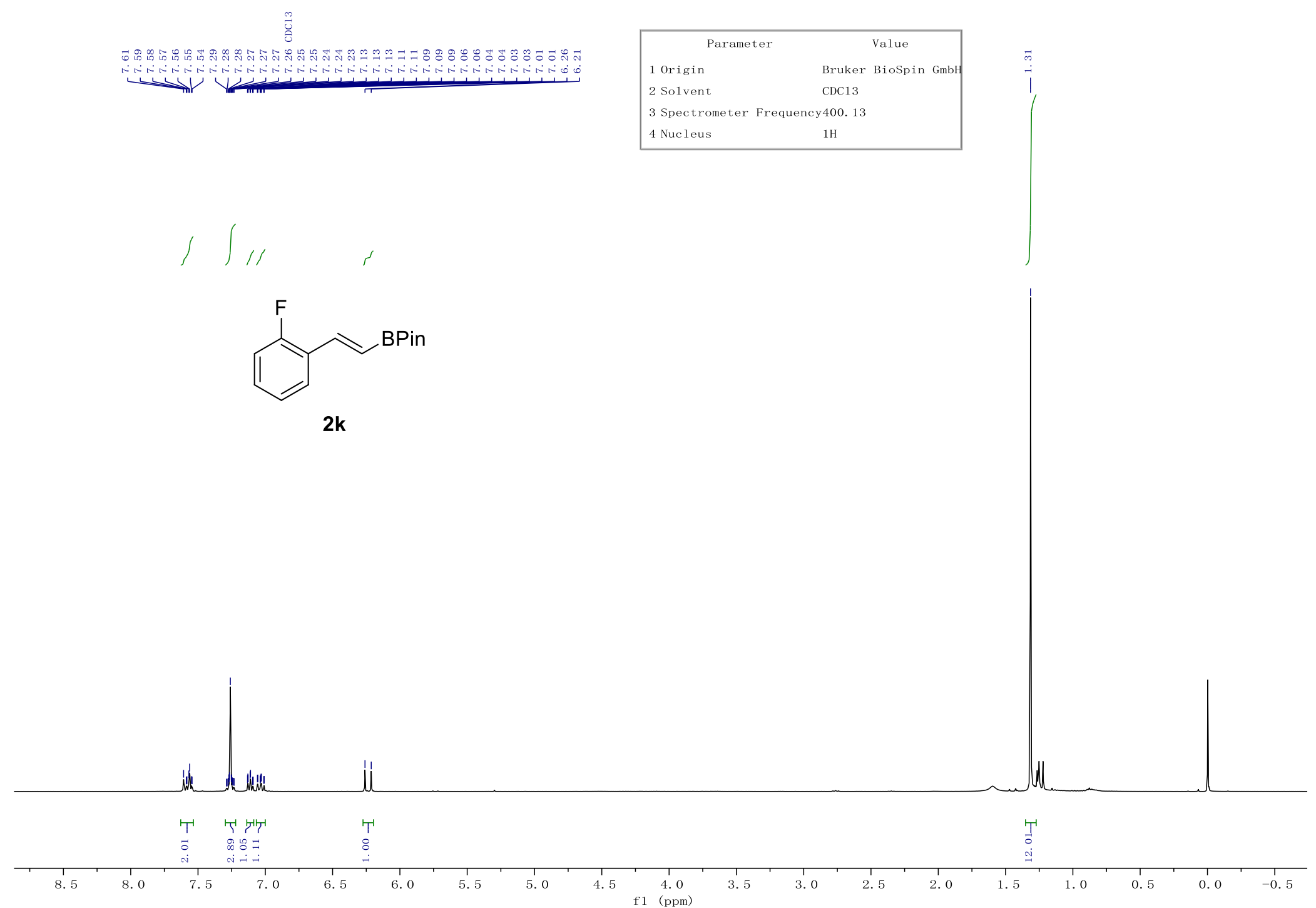




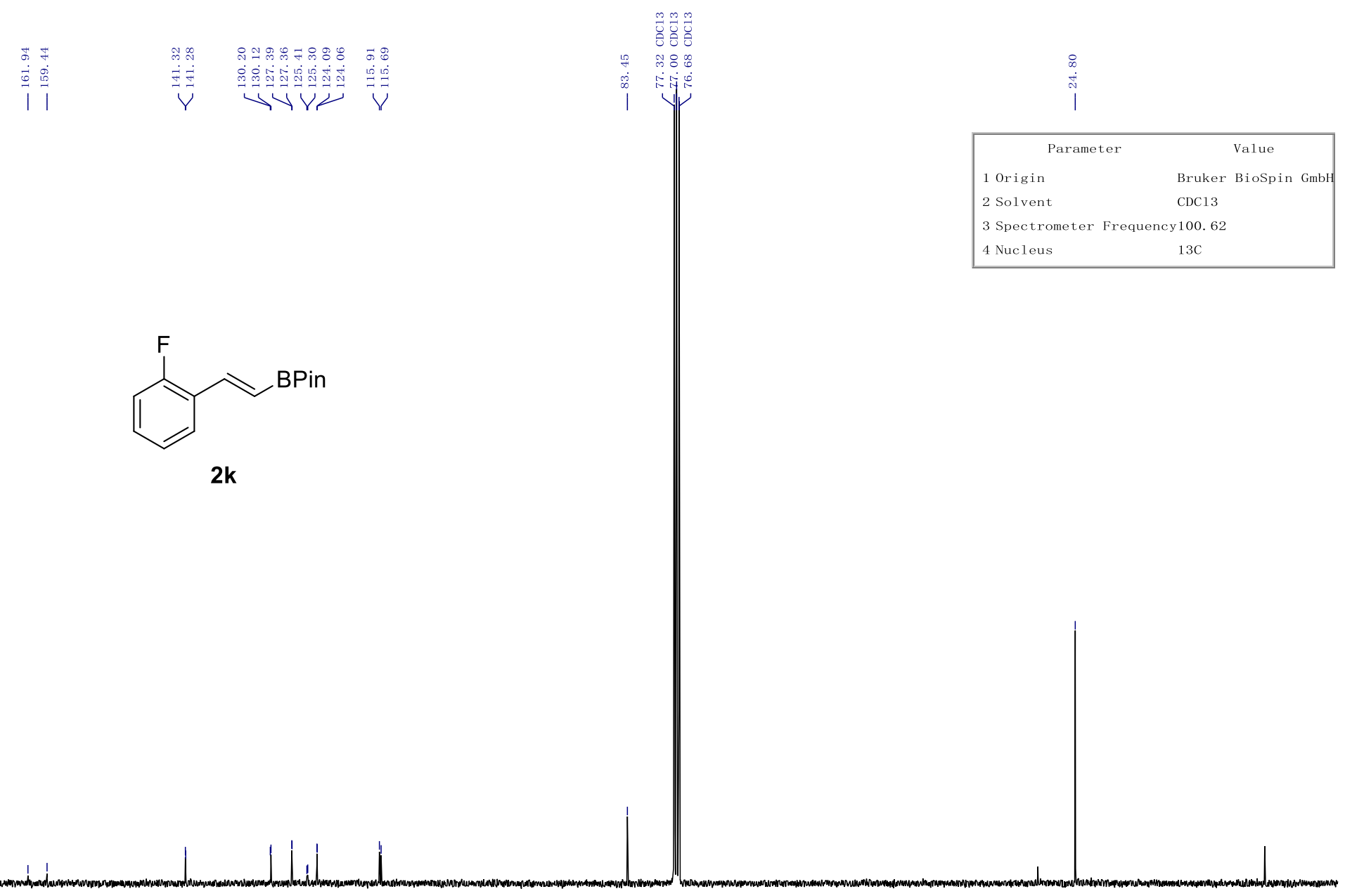

$T_{180}$
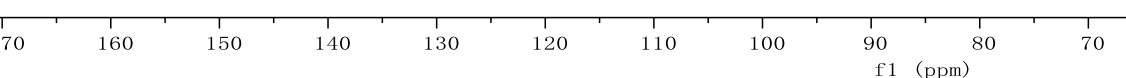


\begin{tabular}{|ll|}
\hline \multicolumn{1}{|c|}{ Parameter } & \multicolumn{2}{c|}{ Value } \\
1 Origin & Bruker BioSpin GmbH \\
2 Solvent & CDC13 \\
3 Spectrometer Frequency376. 46 \\
4 Nucleus & 19F \\
\hline
\end{tabular}
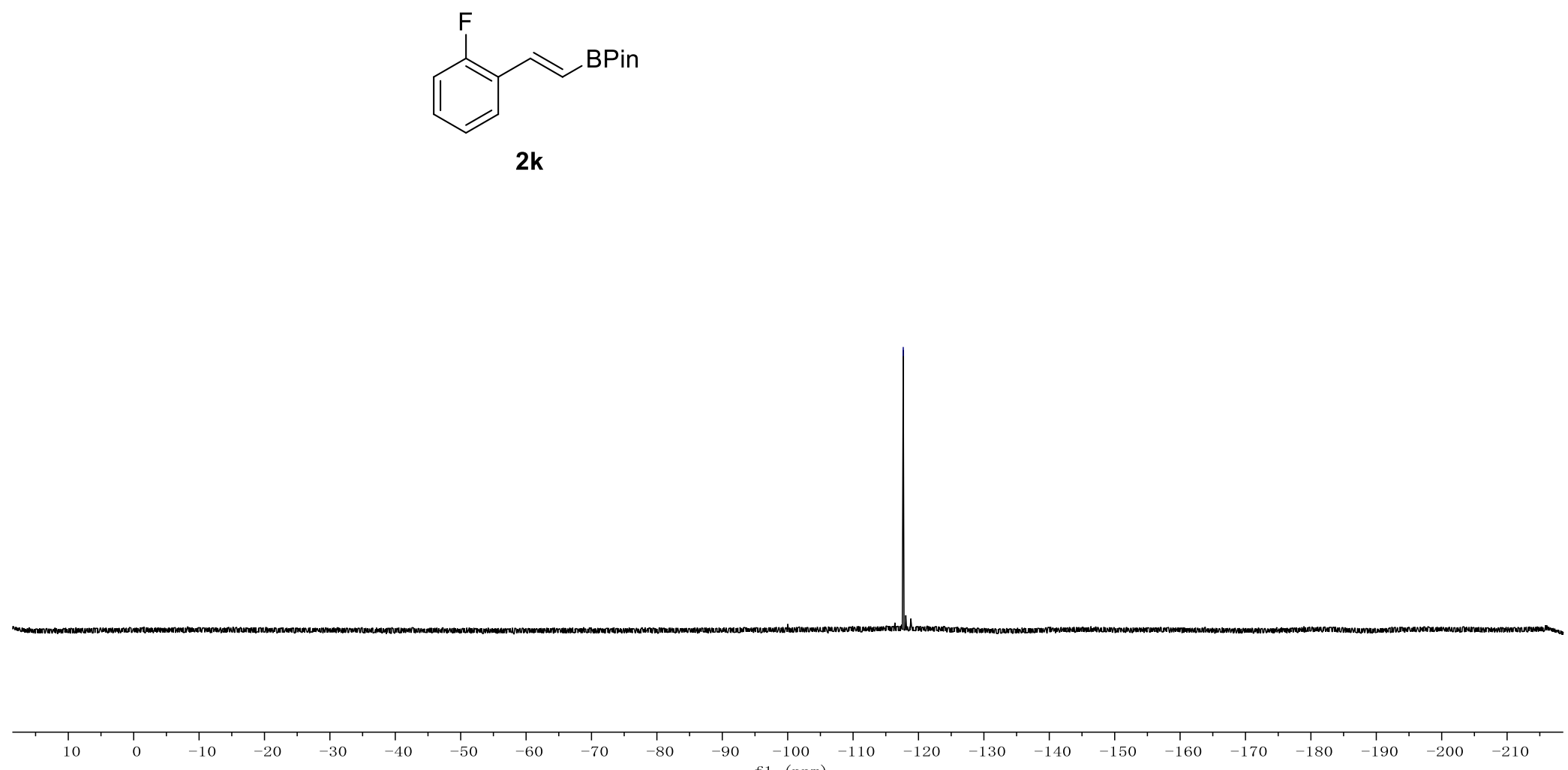


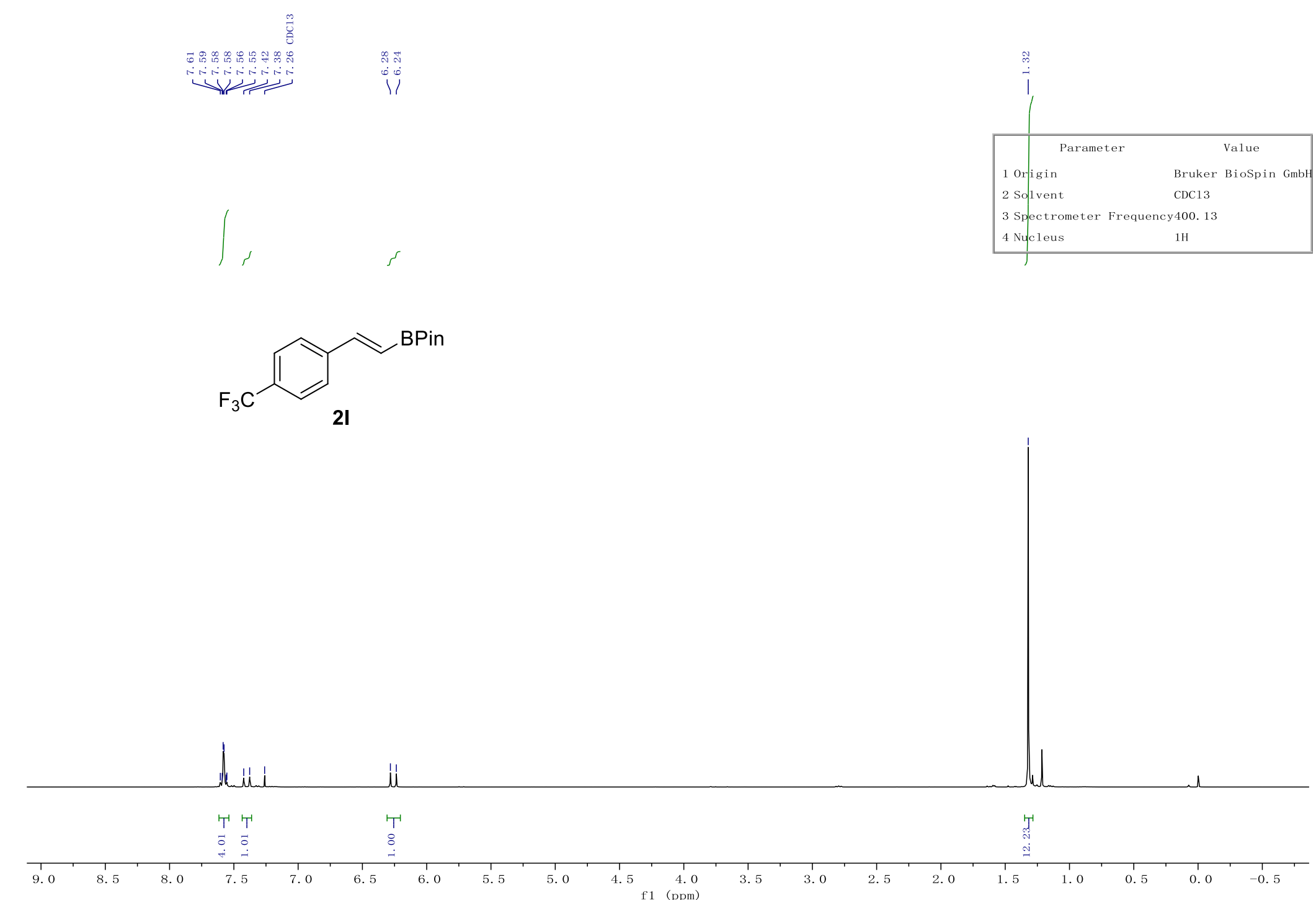



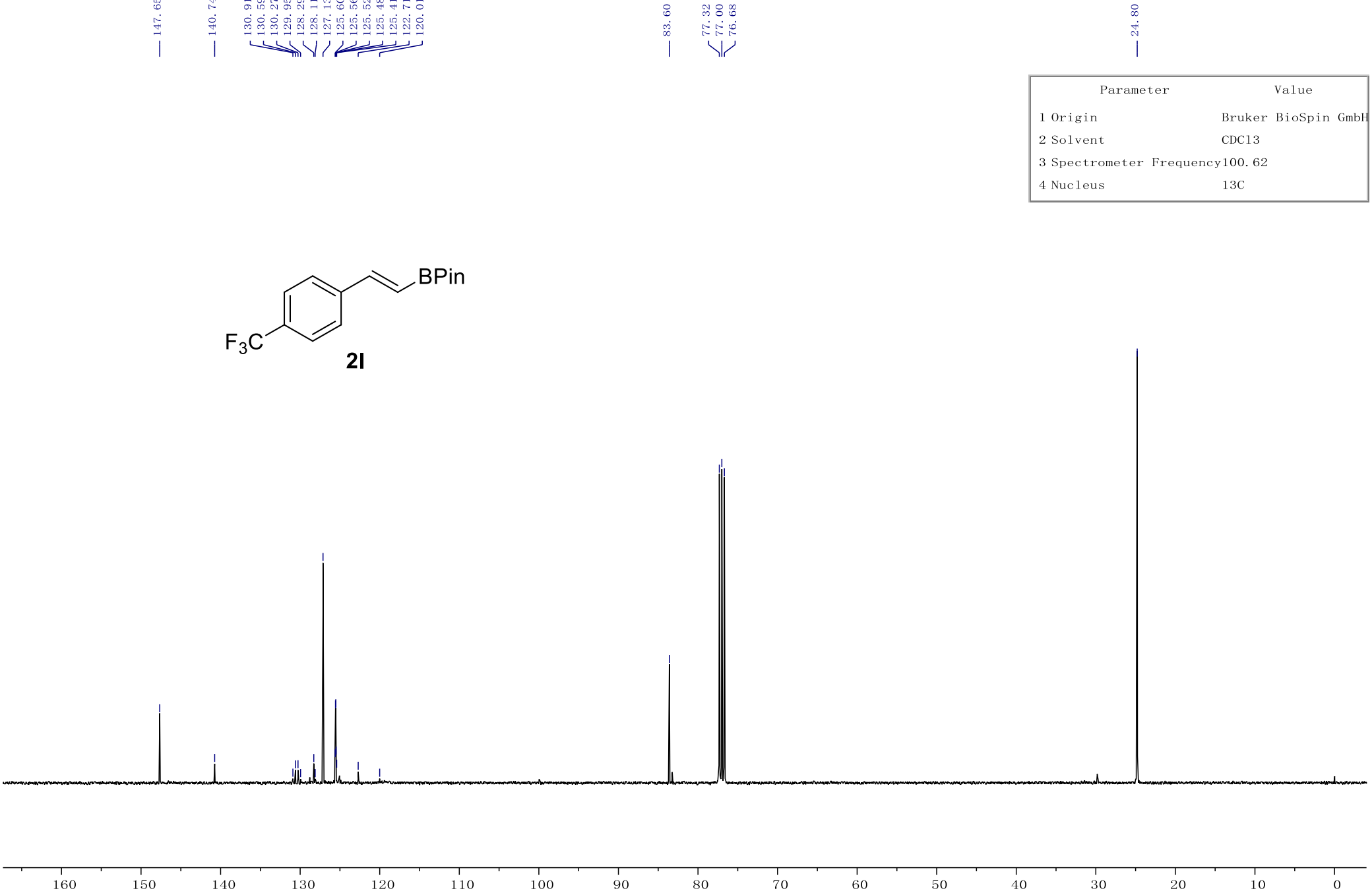

120 f1 $\left(\begin{array}{c}80 \\ \mathrm{ppm})\end{array}\right.$ 


\begin{tabular}{|lc|}
\hline \multicolumn{1}{|c|}{ Parameter } & \multicolumn{1}{c|}{ Value } \\
1 Origin & Bruker \\
2 Solvent & CDC13 \\
3 Spectrometer Frequency376. & \\
4 Nucleus & 19F \\
\hline
\end{tabular}



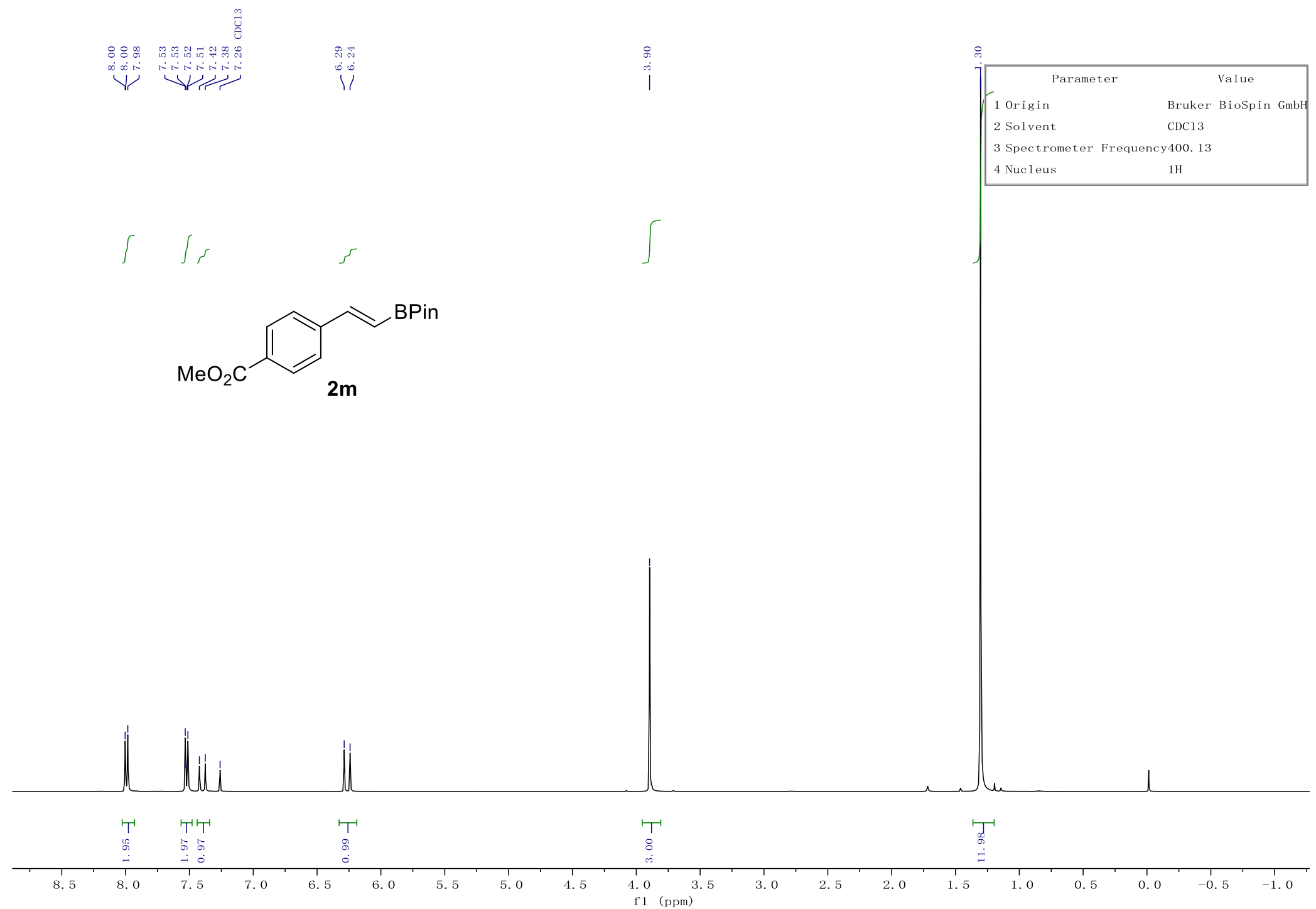






$\mathrm{MeO}_{2} \mathrm{C}$
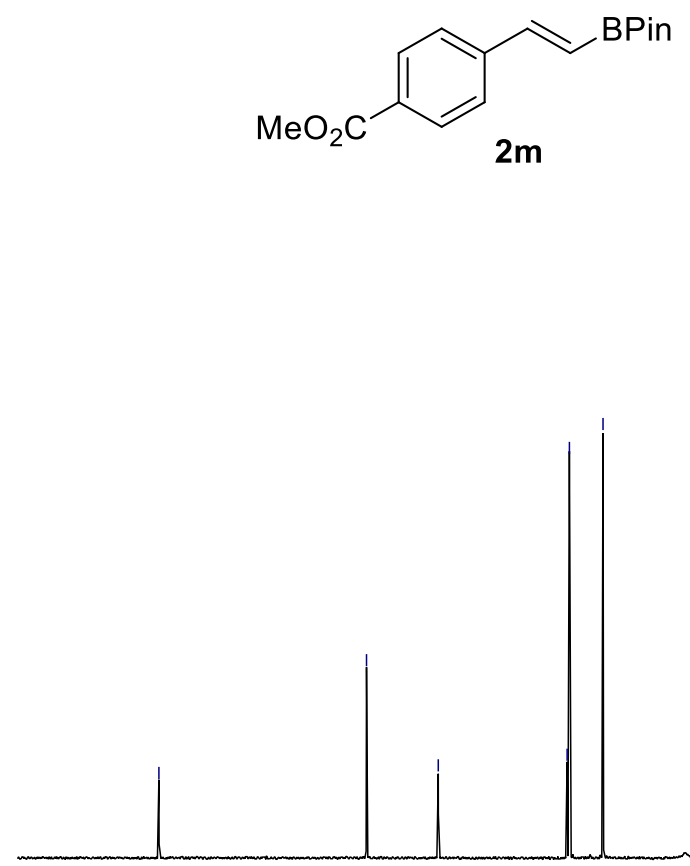
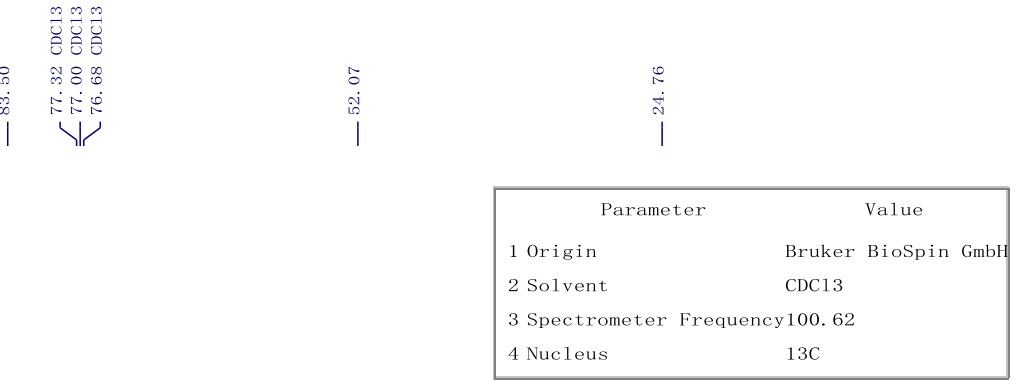

3 Spectr

$13 \mathrm{C}$
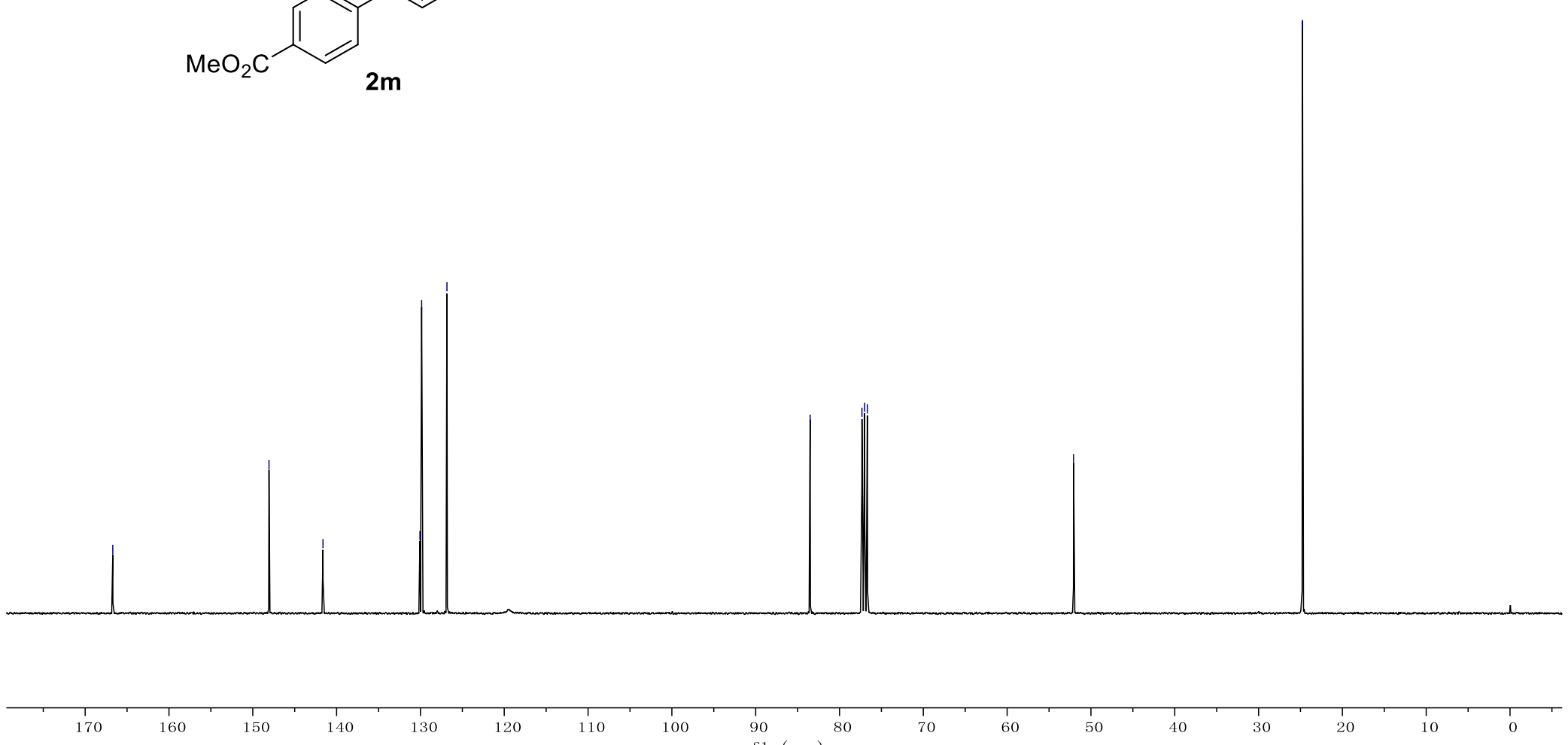

$90 \quad 80$











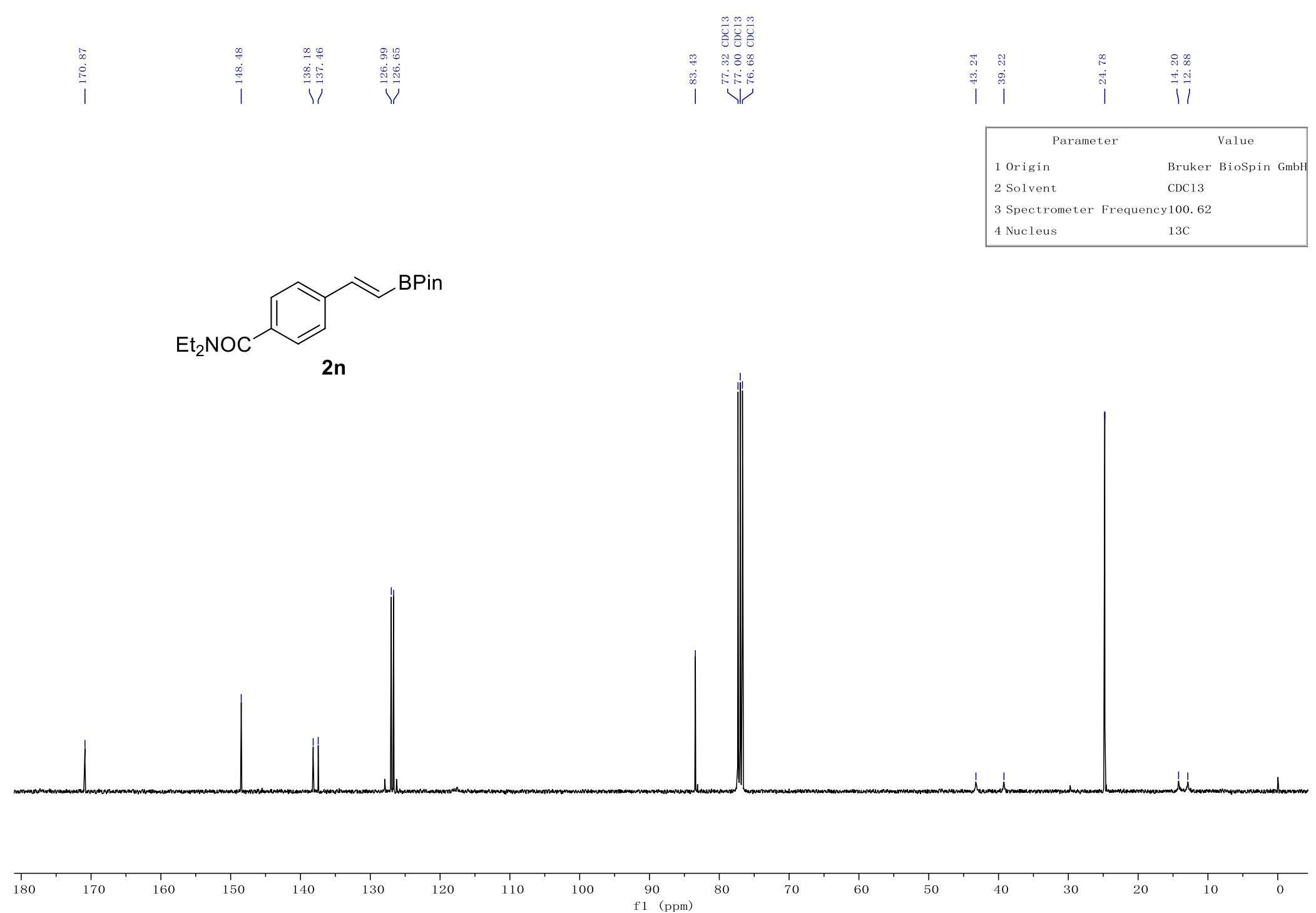




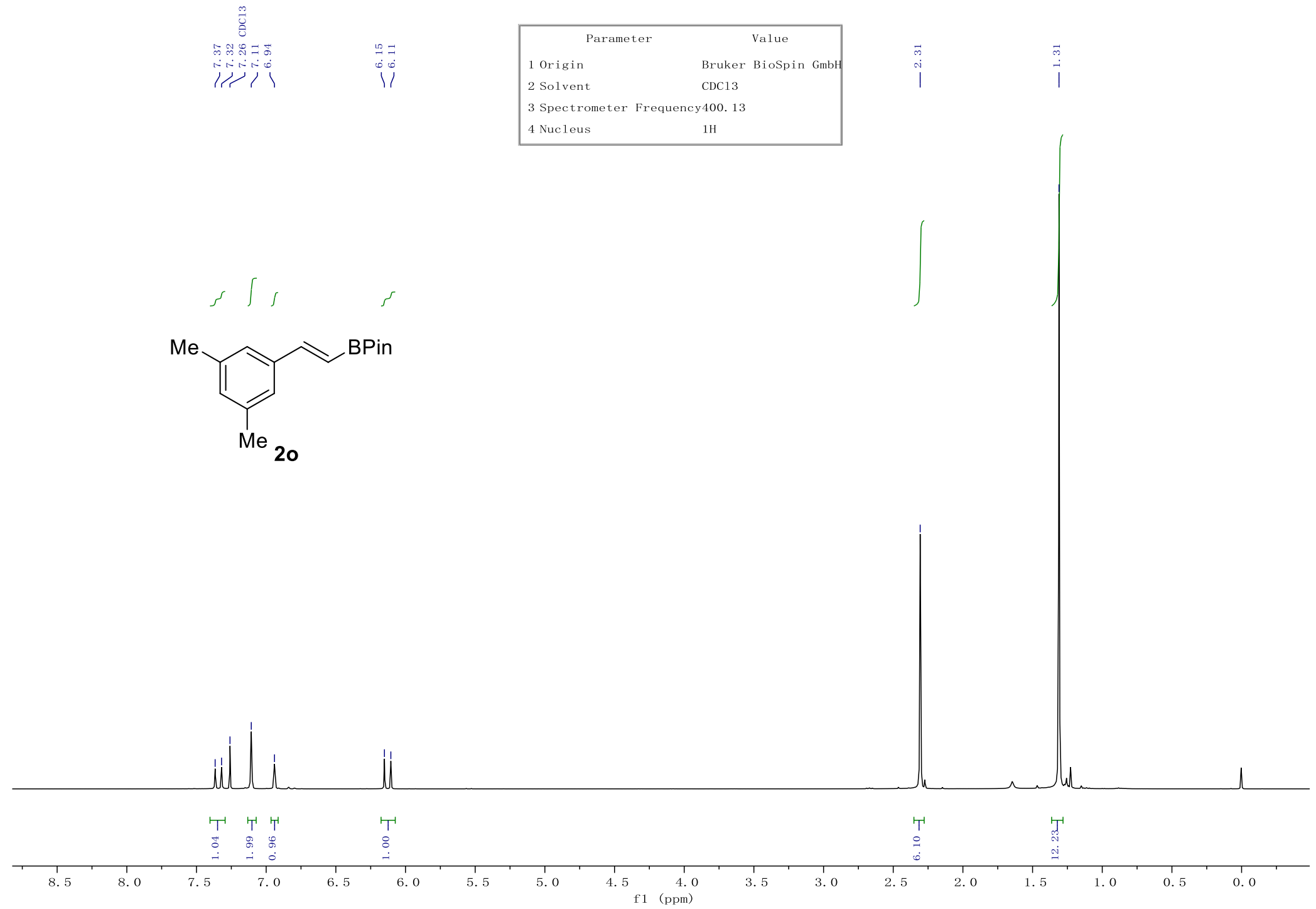




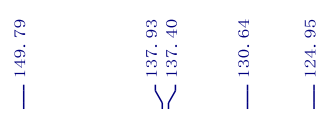

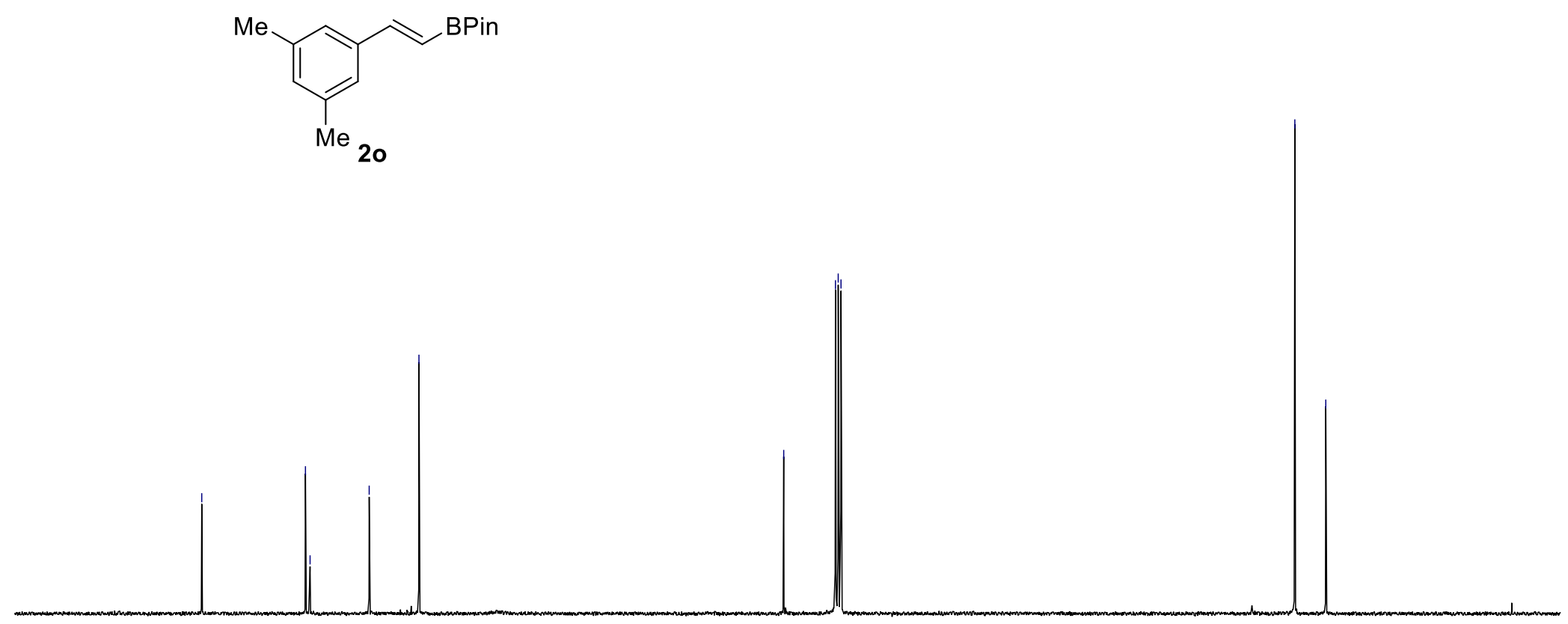

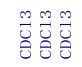

๙

| 농

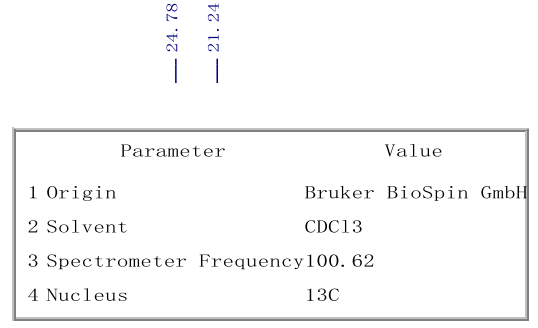

$\frac{1}{170}$

160

150

140


90
90 


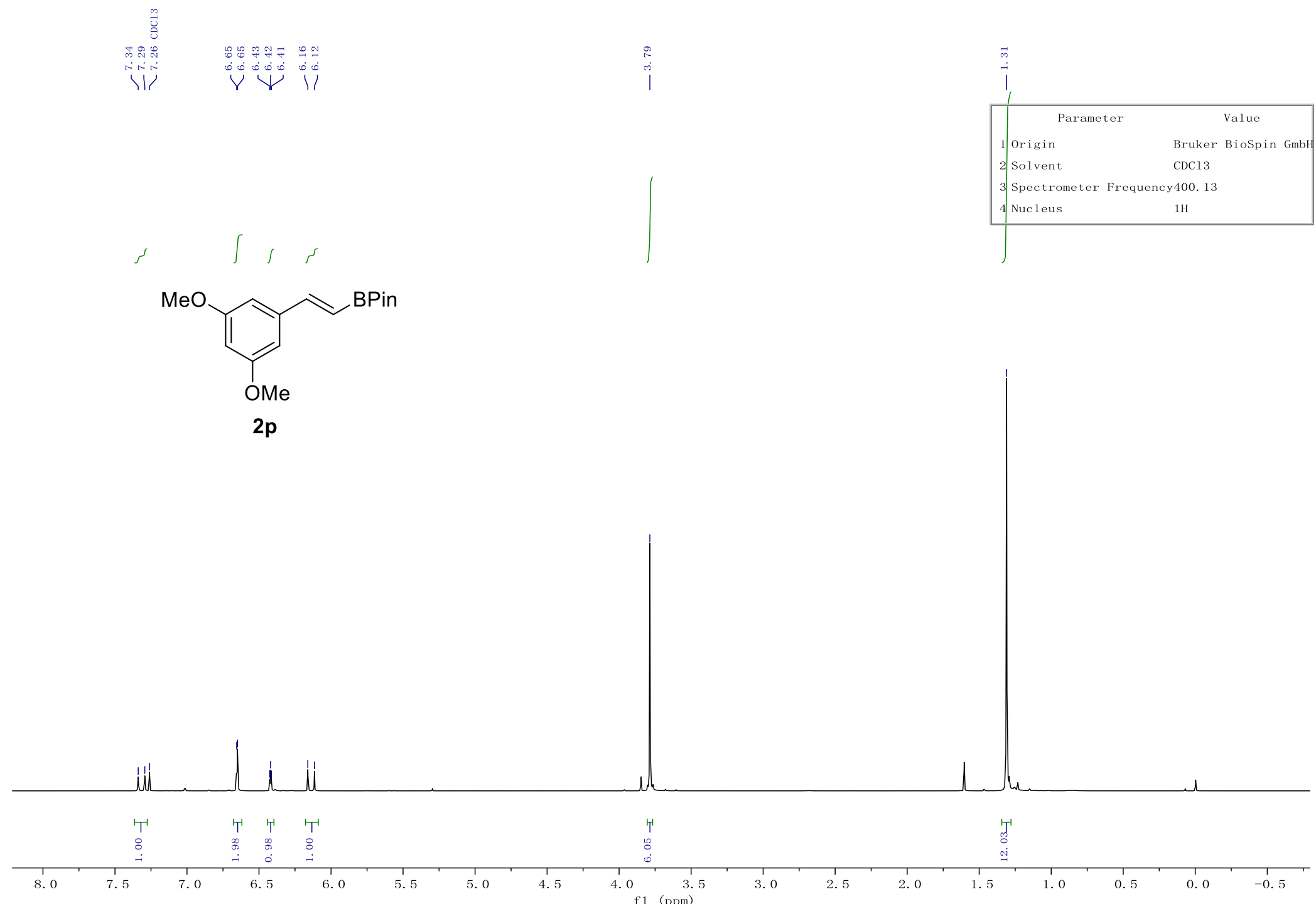



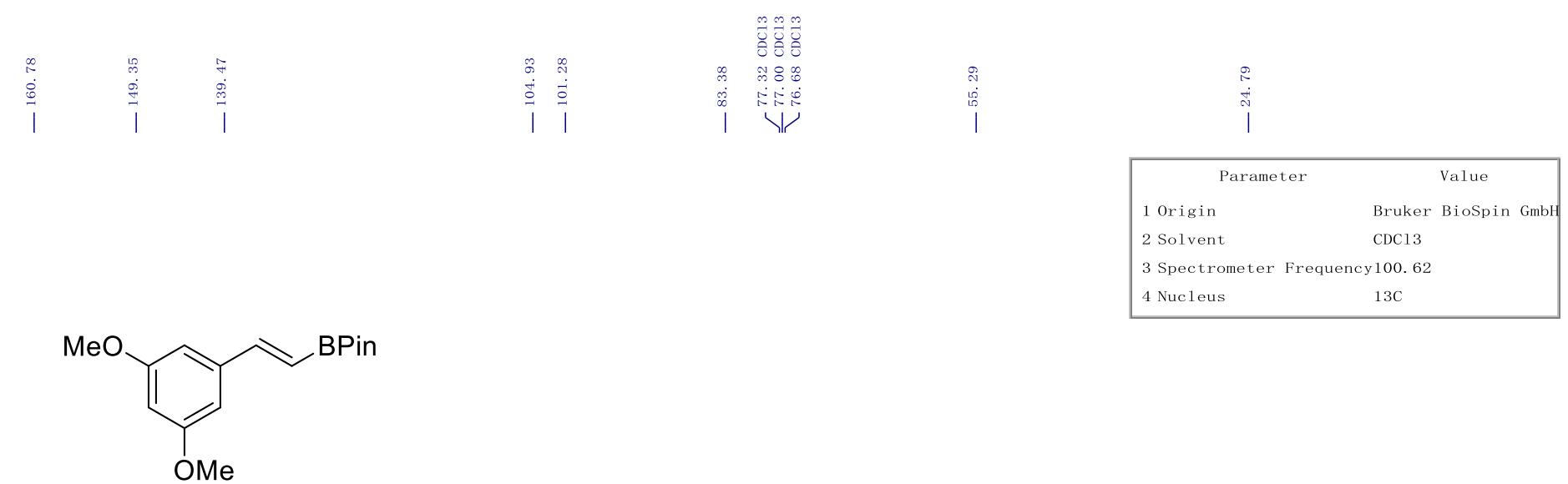

$2 p$

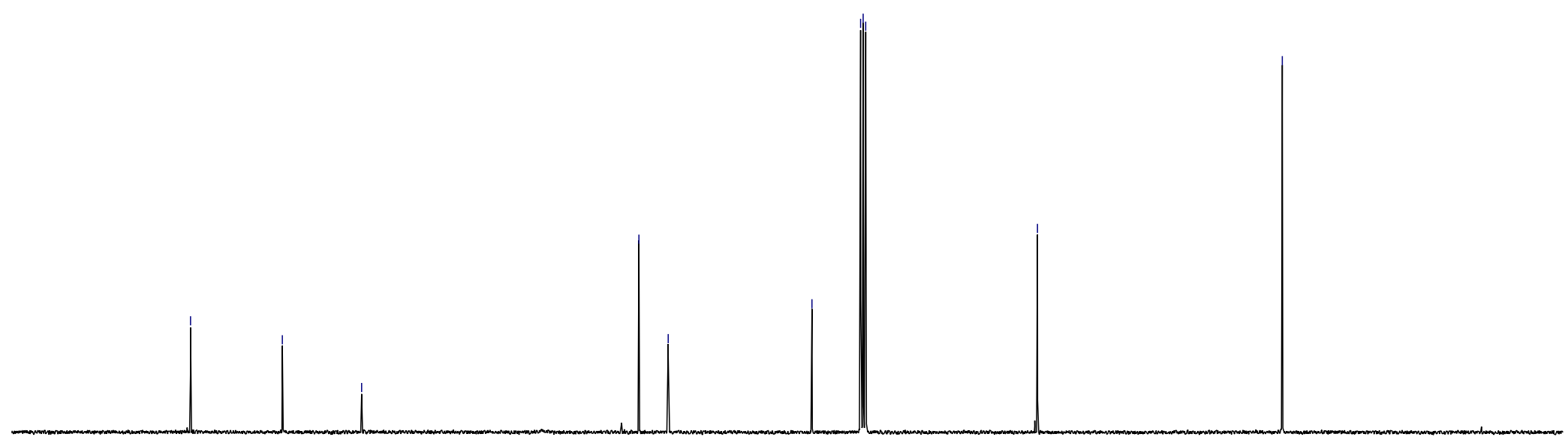

180
180
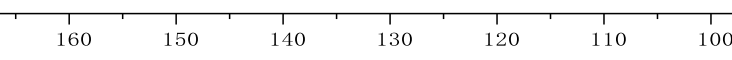

$90 \quad 80$ 


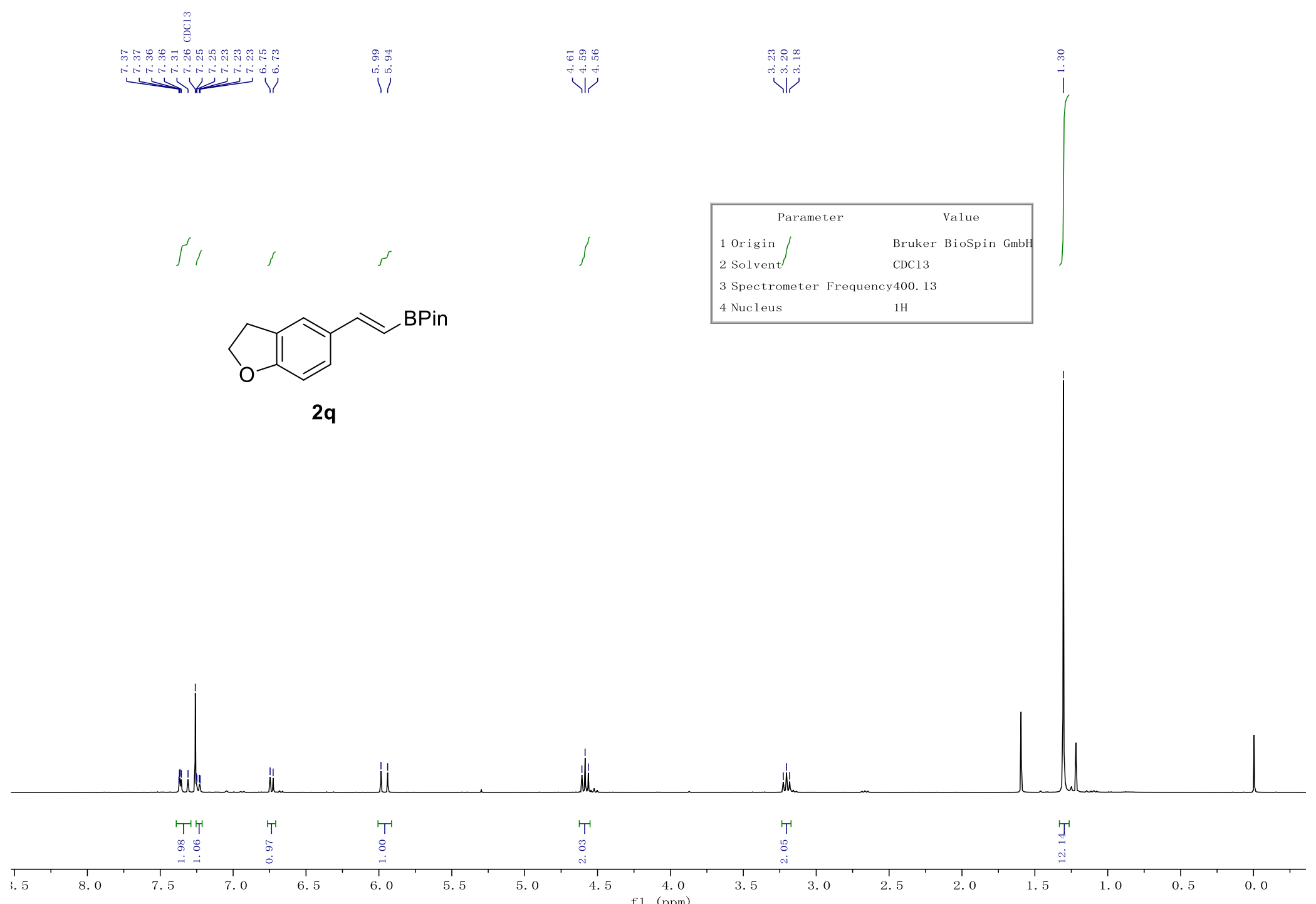









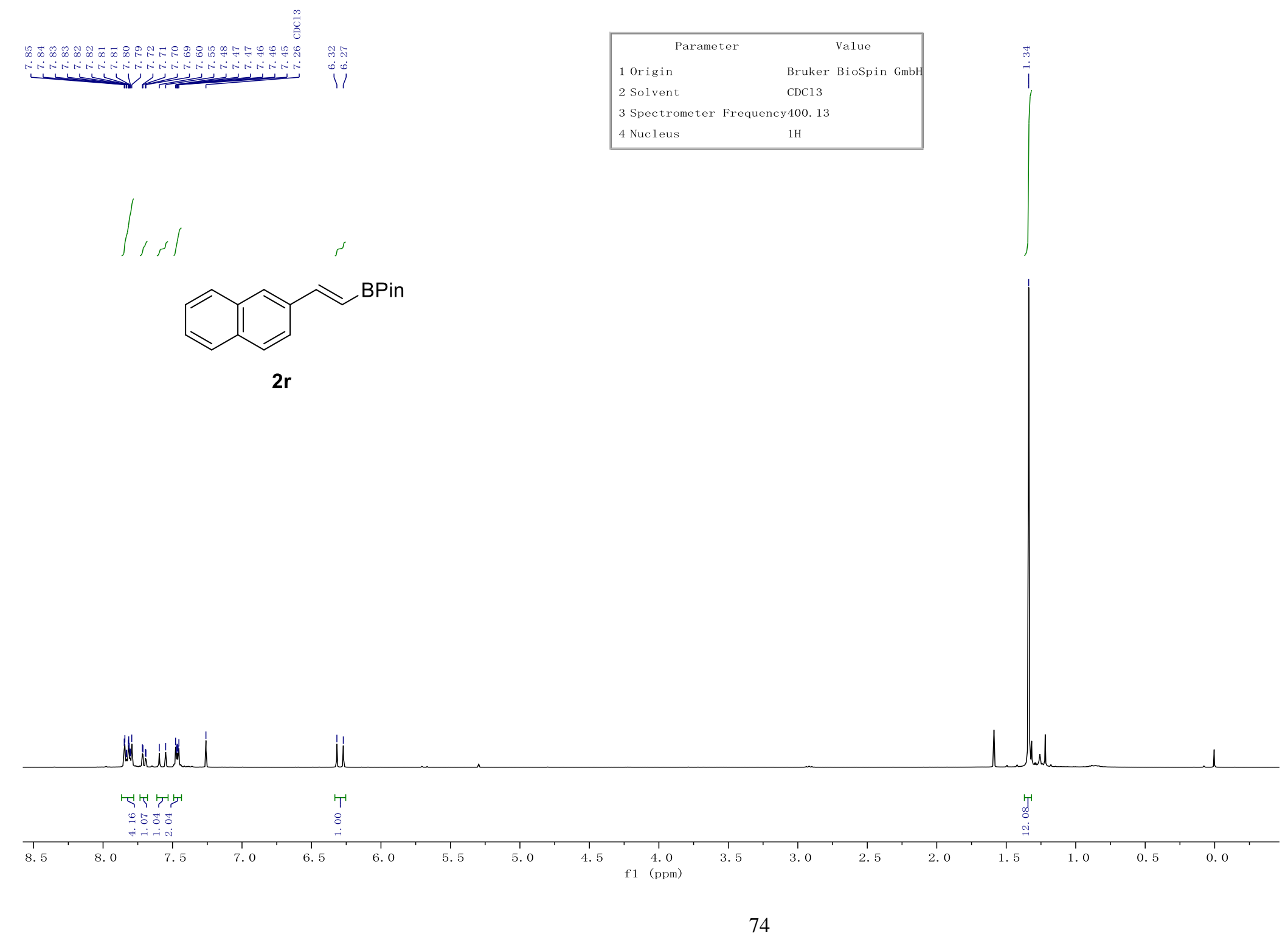









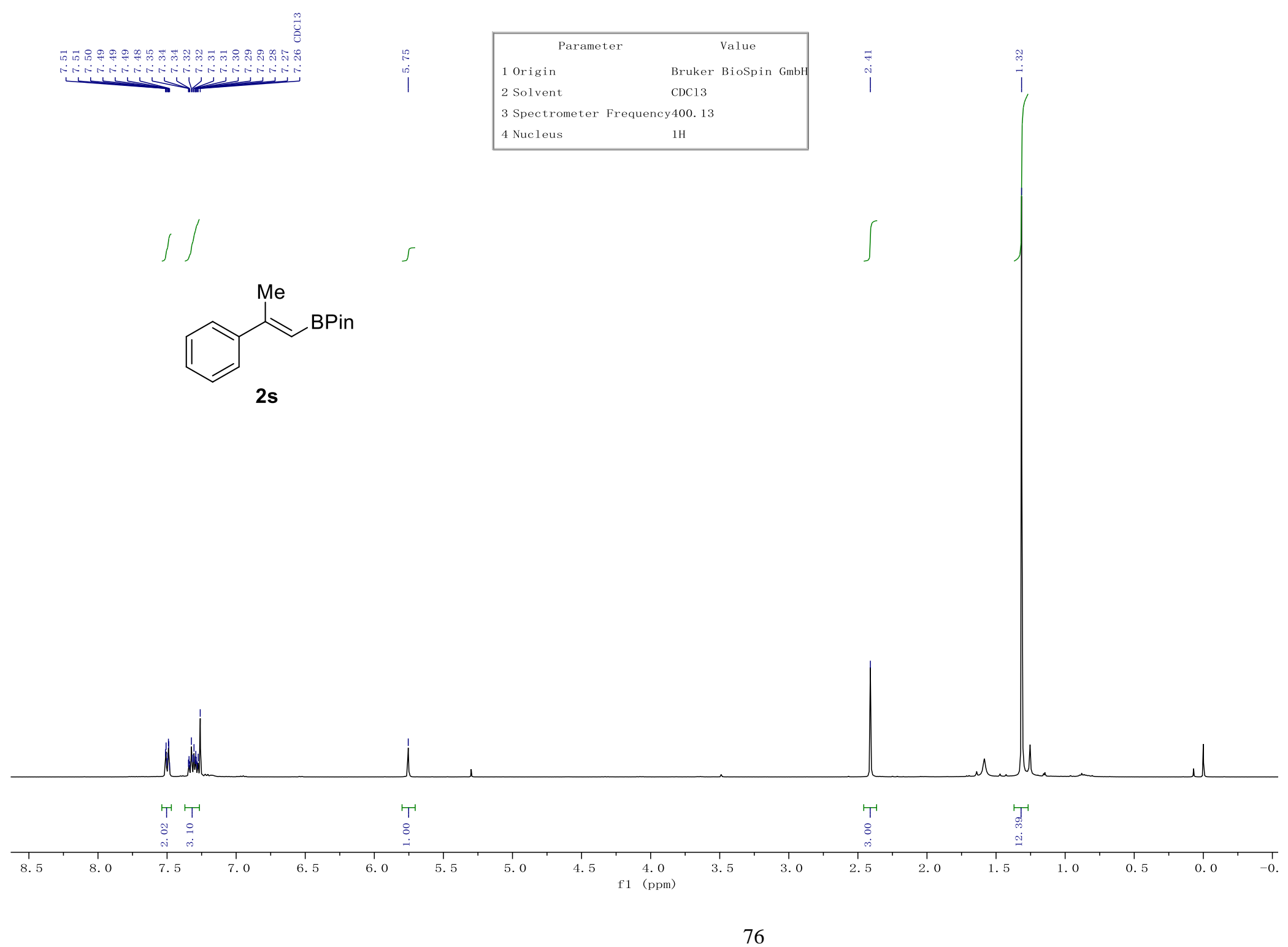







兽

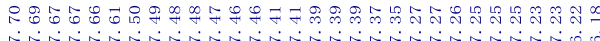

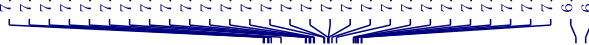
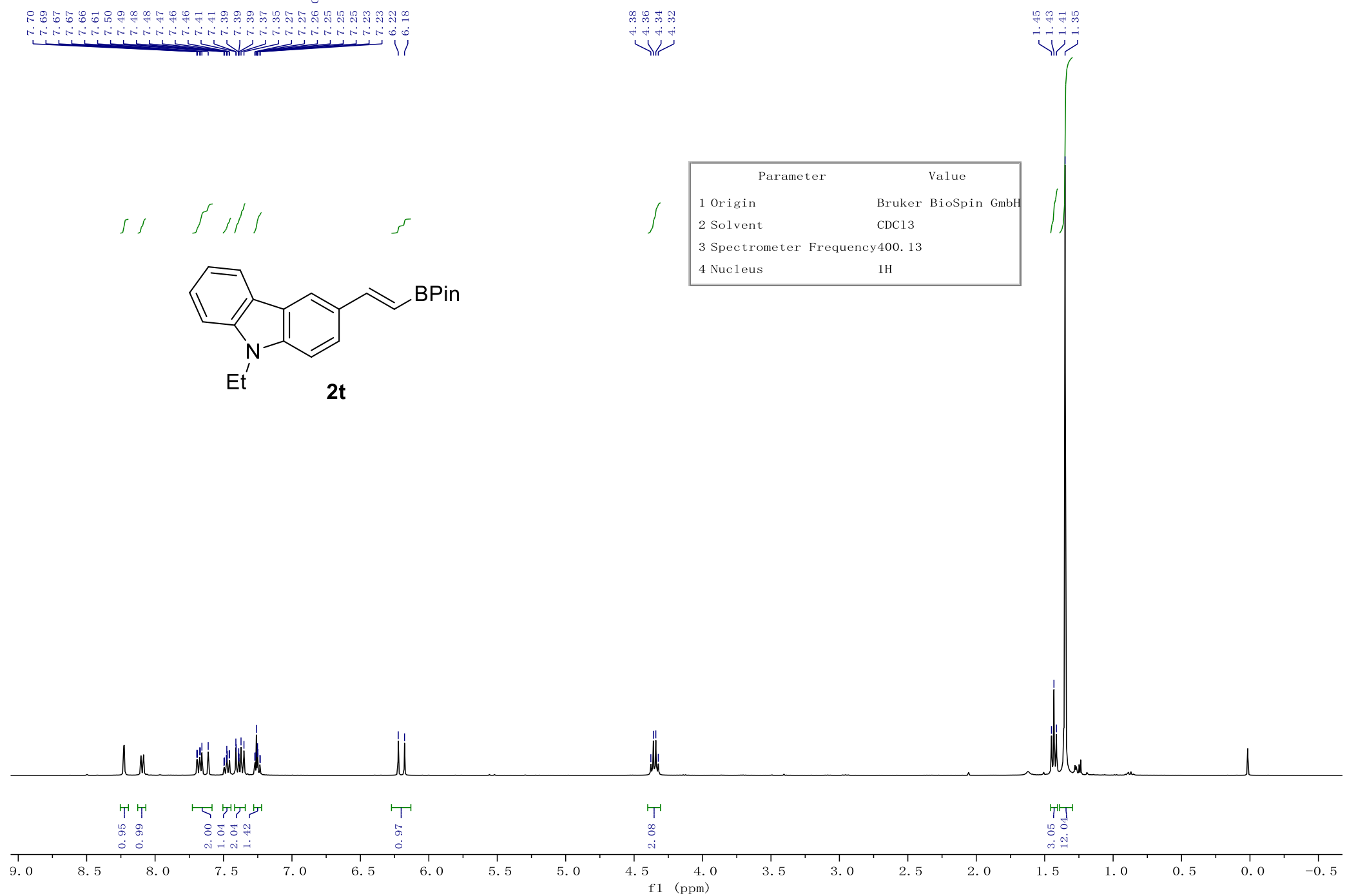


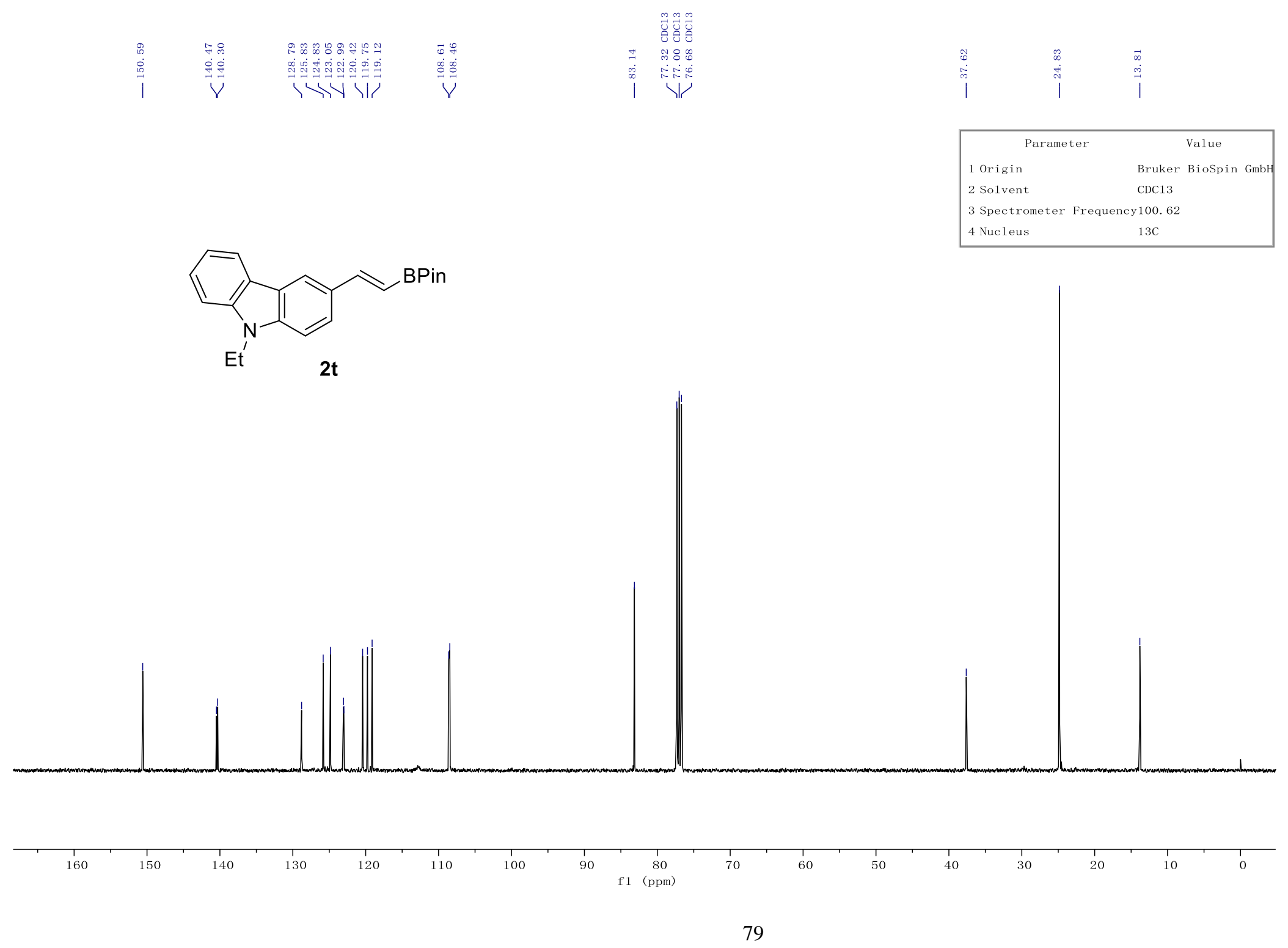


造

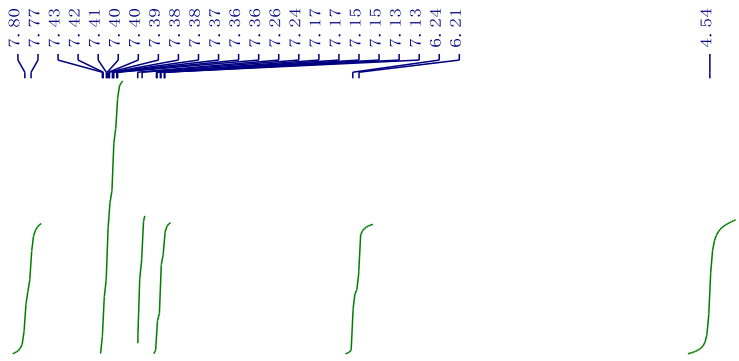

2u

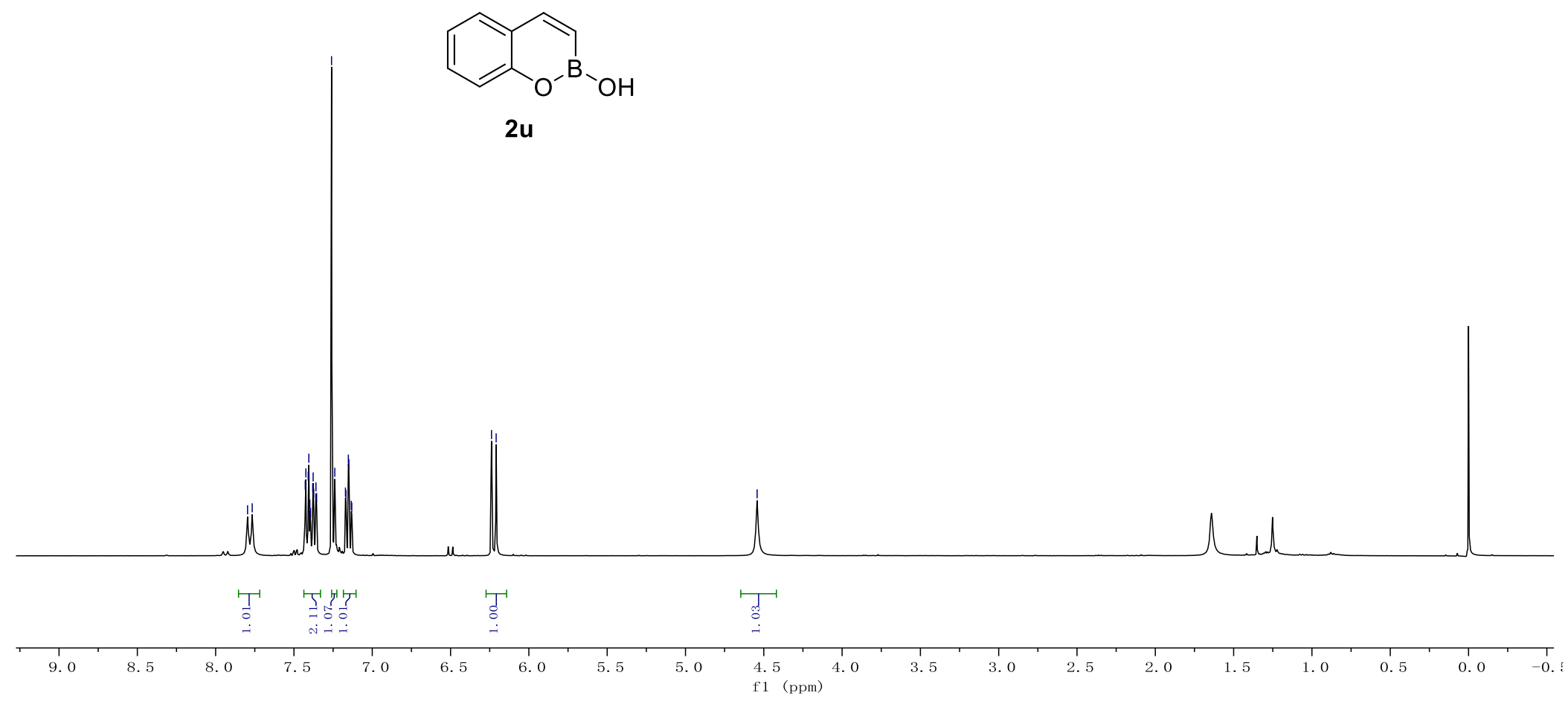

\begin{tabular}{|lc|}
\hline \multicolumn{1}{|c|}{ Parameter } & \multicolumn{1}{c|}{ Value } \\
1 Origin & Bruker \\
2 Solvent & CDC13 \\
3 Spectrometer & Frequency 400.13 \\
4 Nucleus & $1 \mathrm{H}$ \\
\hline
\end{tabular}
$1 \mathrm{H}$ 


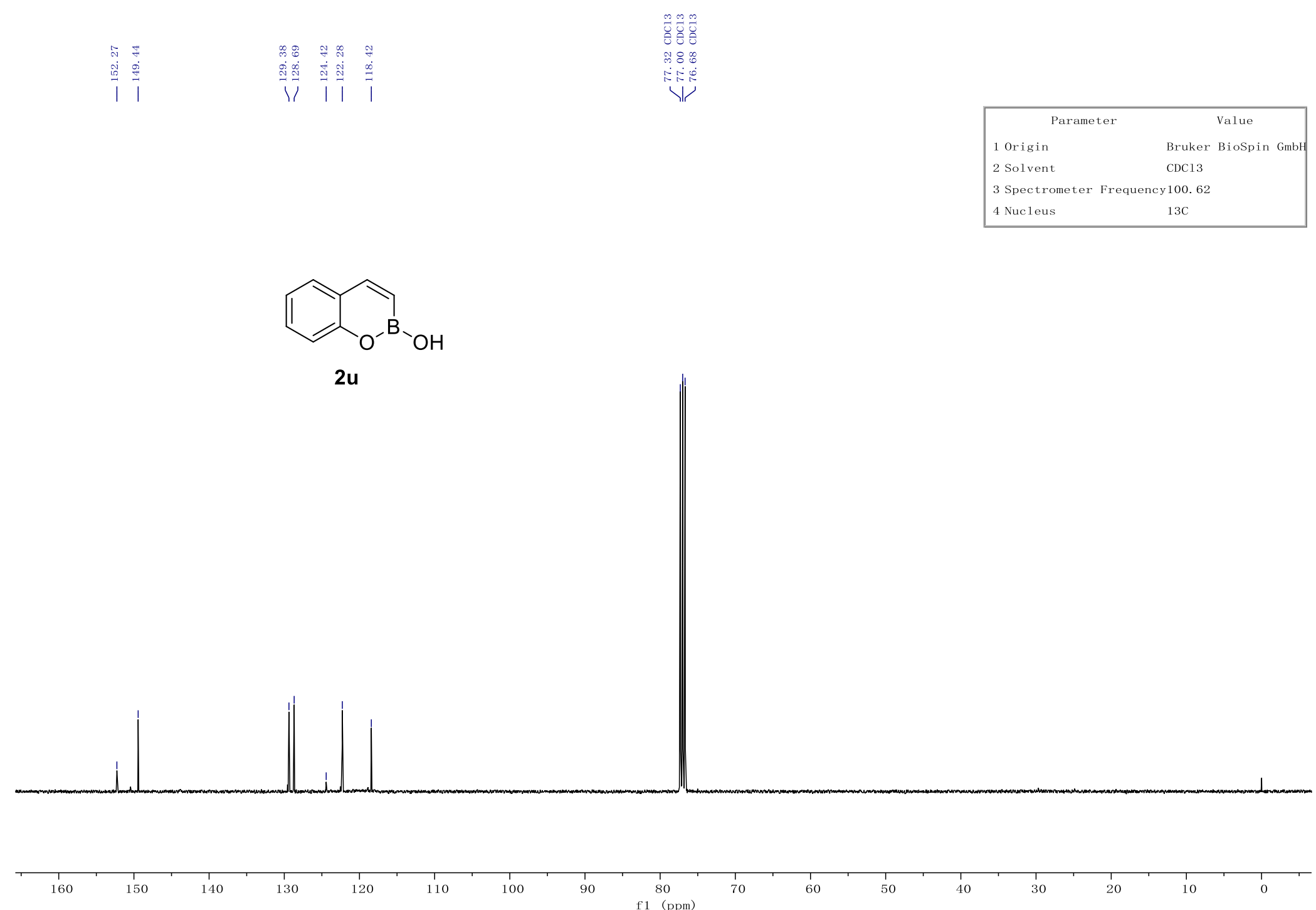




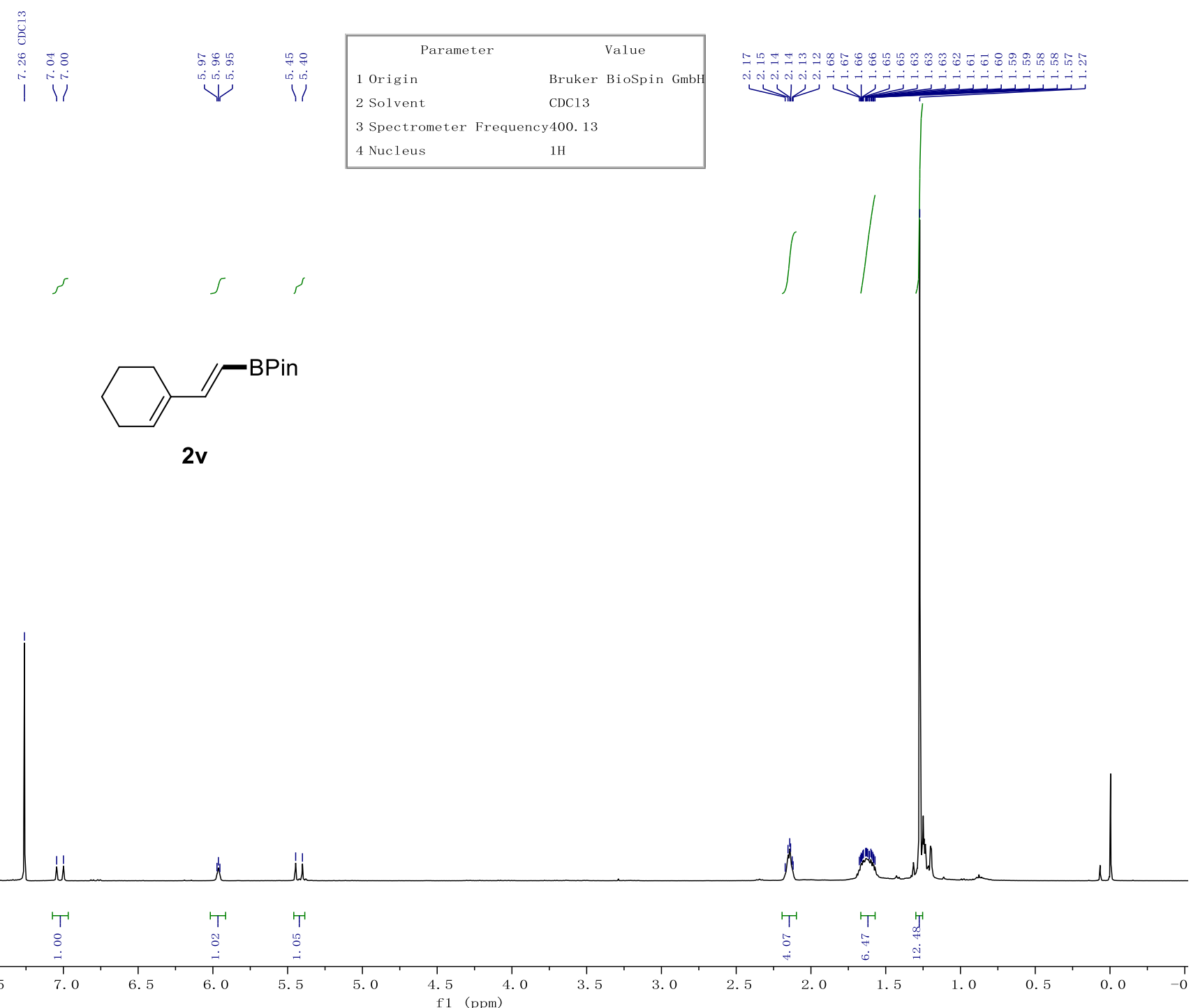






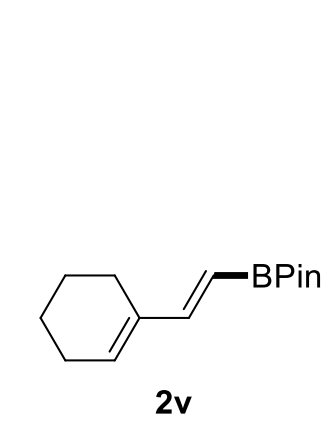

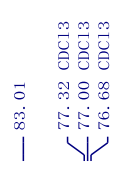

은 도용ㅇ

ปิน

\begin{tabular}{|lc|}
\hline \multicolumn{1}{|c|}{ Parameter } & \multicolumn{1}{c|}{ Value } \\
1 Origin & Bruker BioSpin GmbH \\
2 Solvent & CDC13 \\
3 Spectrometer Frequency 100. 62 \\
4 Nucleus & $13 \mathrm{C}$ \\
\hline
\end{tabular}






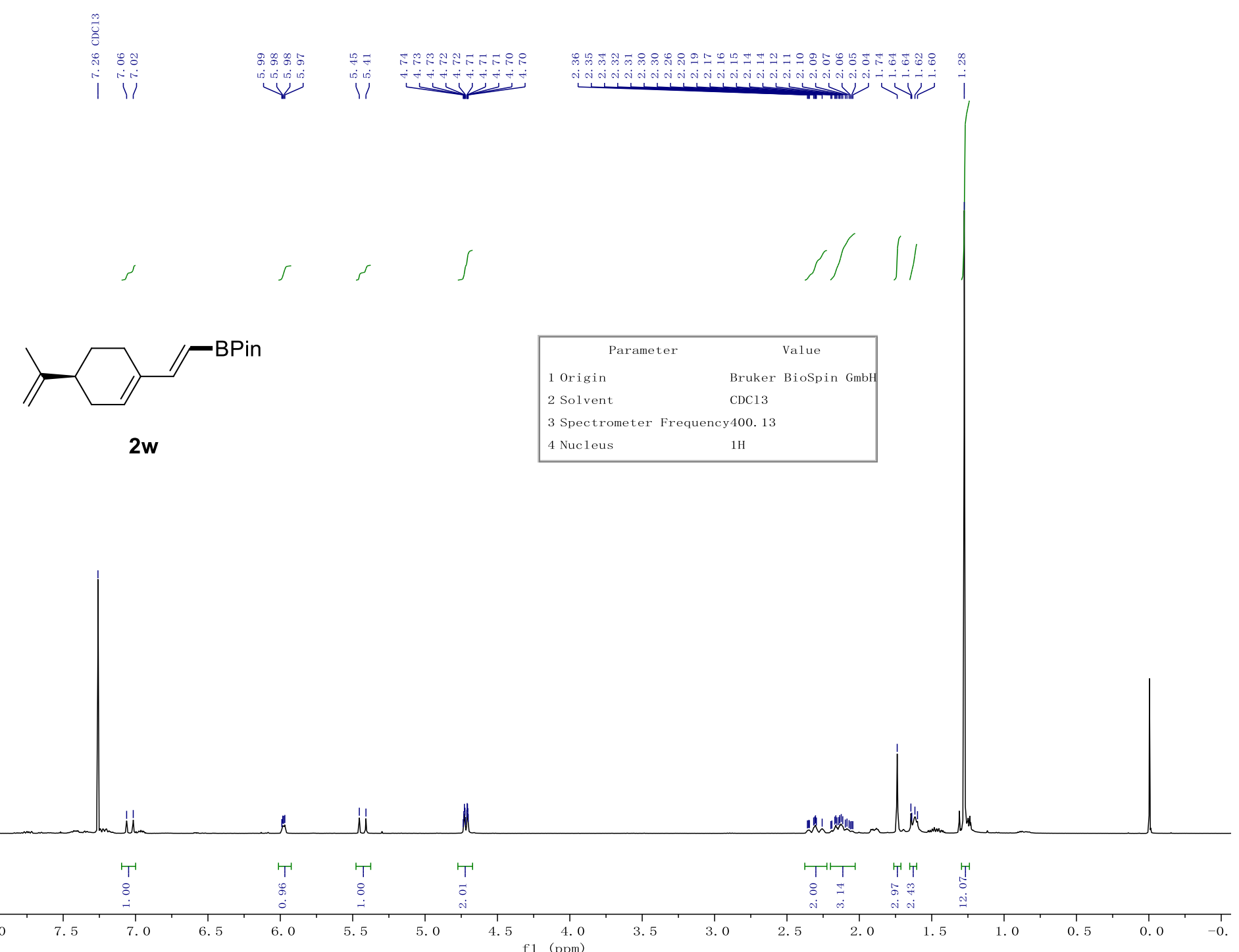



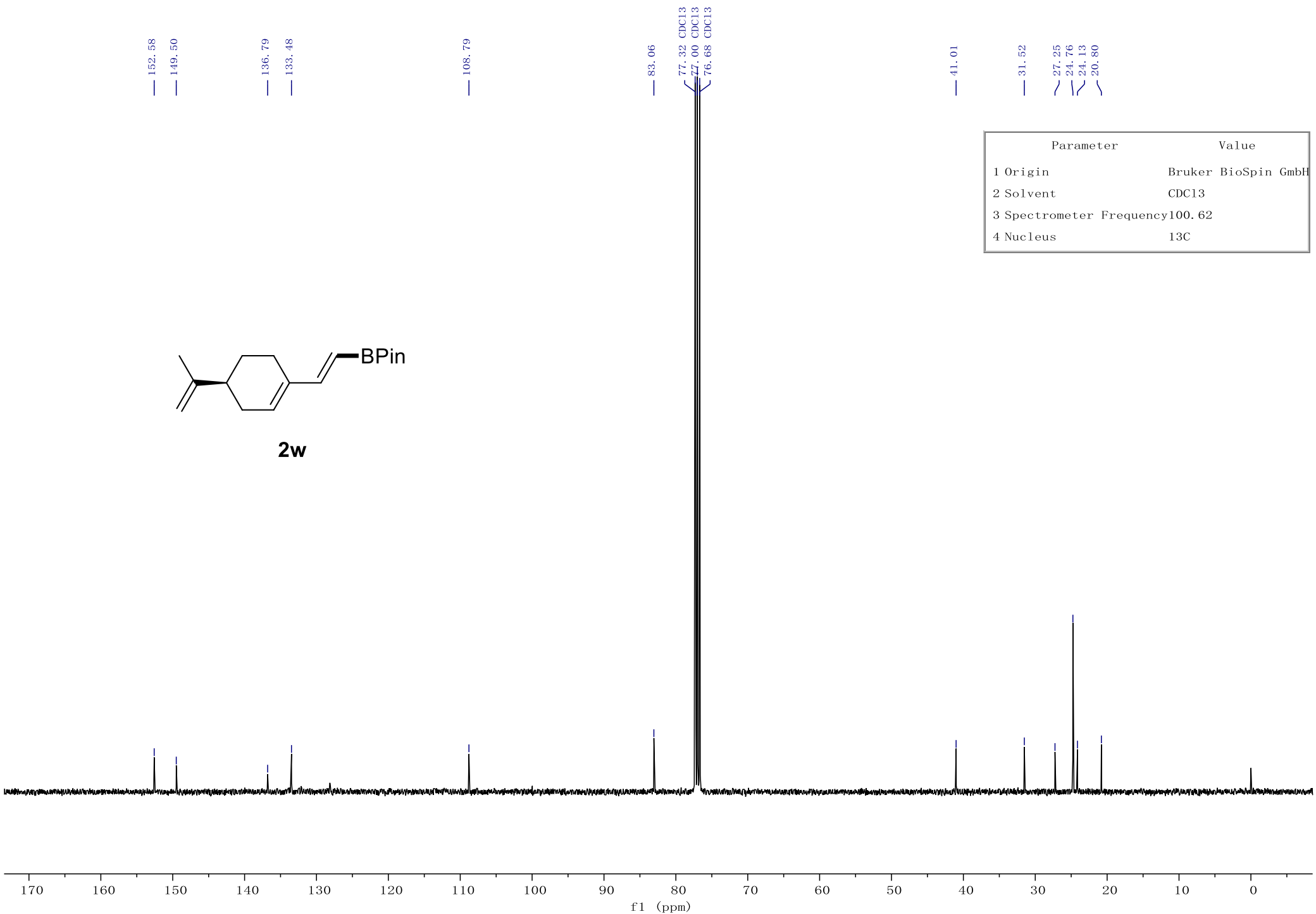


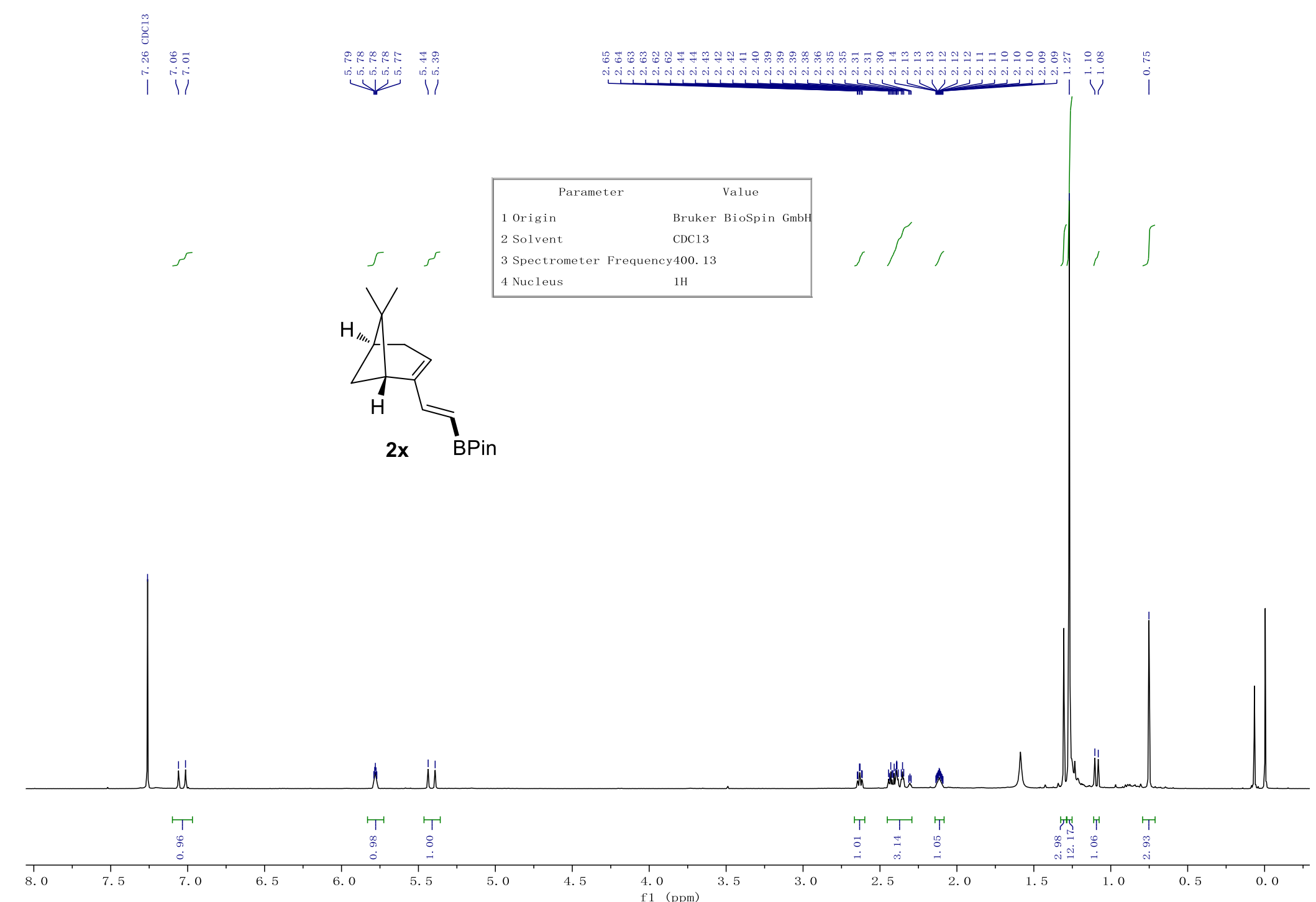







莺

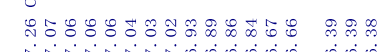




ivilith

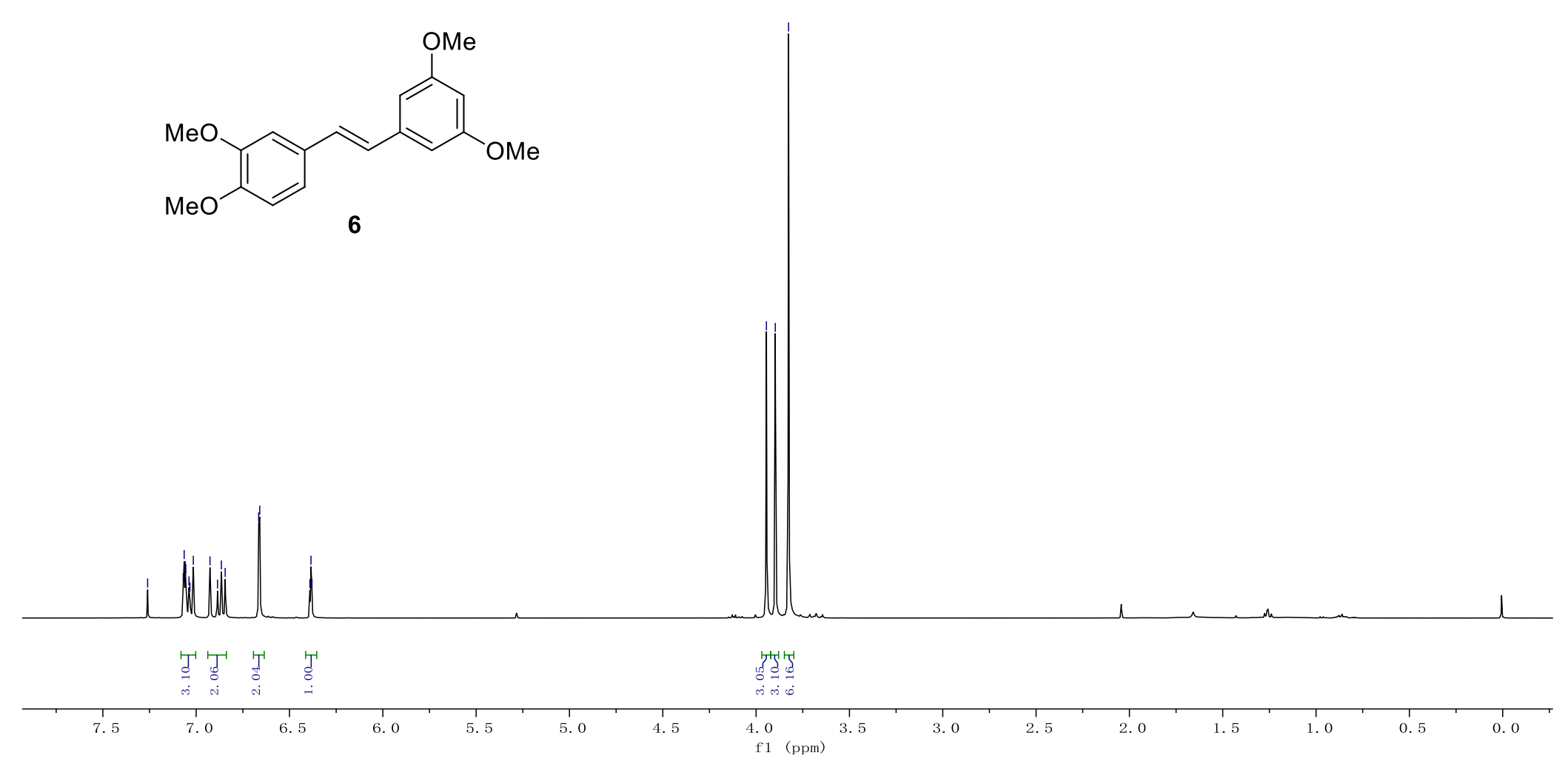

\begin{tabular}{|ll|}
\hline \multicolumn{1}{|c|}{ Parameter } & \multicolumn{1}{c|}{ Value } \\
1 Origin & Bruker BioSpin GmbH \\
2 Solvent & $\mathrm{CDC13}$ \\
3 Spectrometer Frequency 400.13 \\
4 Nucleus & $1 \mathrm{H}$ \\
\hline
\end{tabular}

4 Nucleus $1 \mathrm{H}$ 


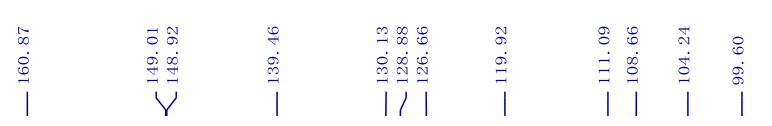



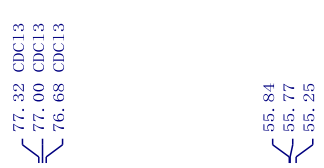

\begin{tabular}{|lc|}
\hline \multicolumn{1}{|c|}{ Parameter } & \multicolumn{1}{c|}{ Value } \\
1 Origin & Bruker BioSpin GmbH \\
2 Solvent & CDC13 \\
3 Spectrometer Frequency100. 62 \\
4 Nucleus & $13 \mathrm{C}$ \\
\hline
\end{tabular}



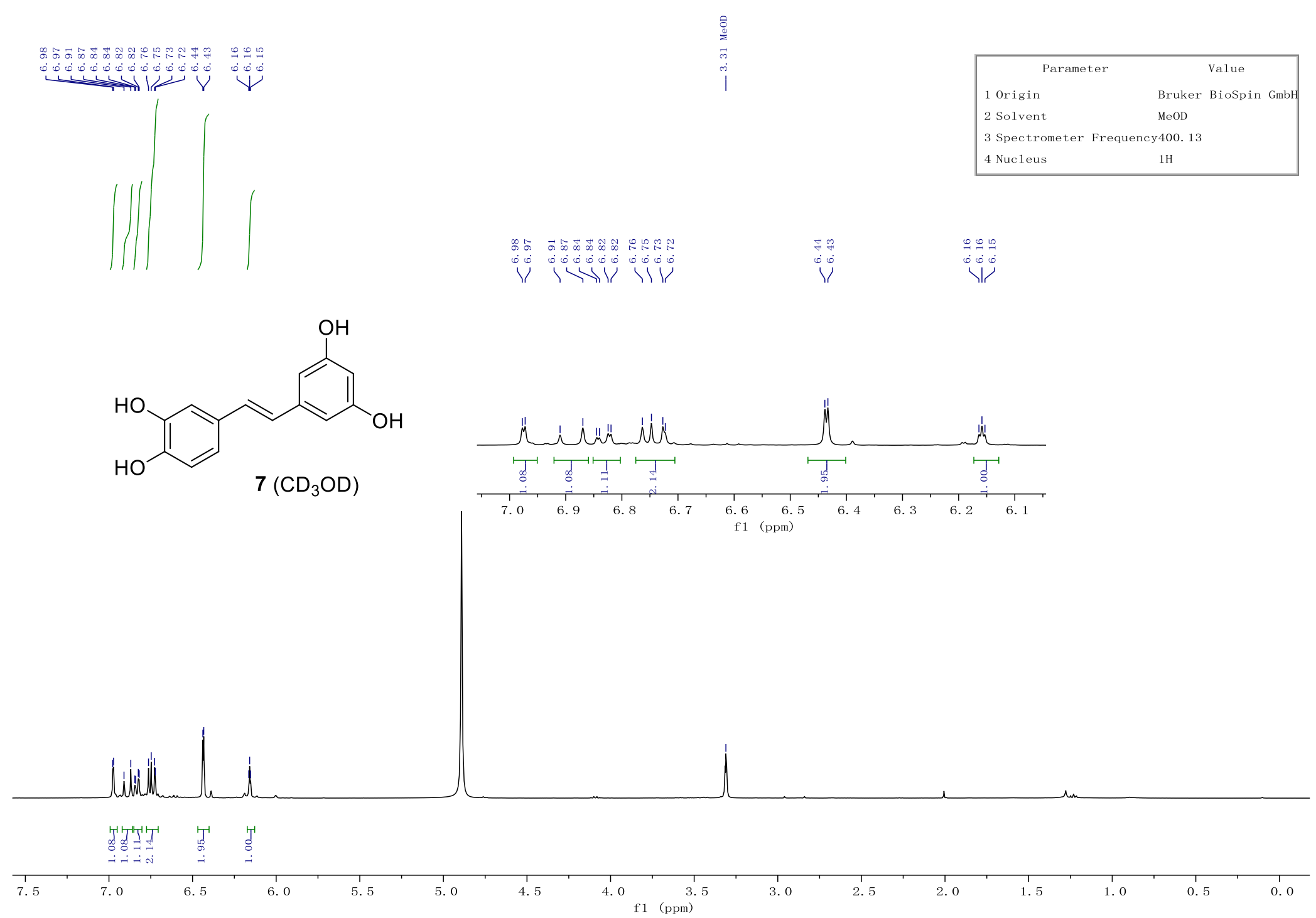

90 


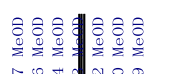

5.9

\begin{tabular}{|ll|}
\hline \multicolumn{1}{|c|}{ Parameter } & \multicolumn{1}{c|}{ Value } \\
1 Origin & Bruker BioSpin GmbH \\
2 Solvent & MeOD \\
3 Spectrometer & Frequency 100. 62 \\
4 Nucleus & $13 C$ \\
\hline
\end{tabular}<smiles>Oc1cc(O)cc(/C=C/c2ccc(O)c(O)c2)c1</smiles>



$\lcm{70}$ 160 150

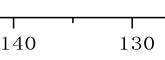

120 f1 $\begin{array}{r}80 \\ \text { (ppm) }\end{array}$ 